\title{
A Hard X-Ray Study of the Normal Star-Forming Galaxy M83 with NuSTAR
}

Yukita, M.; Hornschemeier, A. E.; Lehmer, B. D.; Ptak, A.; Wik, D. R.; Zezas, A.; Antoniou, V.; Maccarone, T. J.; Replicon, V.; Tyler, J. B.

Total number of authors:

22

Published in:

Astrophysical Journal

Link to article, DOI:

$10.3847 / 0004-637 X / 824 / 2 / 107$

Publication date:

2016

Document Version

Publisher's PDF, also known as Version of record

Link back to DTU Orbit

Citation (APA):

Yukita, M., Hornschemeier, A. E., Lehmer, B. D., Ptak, A., Wik, D. R., Zezas, A., Antoniou, V., Maccarone, T. J., Replicon, V., Tyler, J. B., Venters, T., Argo, M. K., Bechtol, K., Boggs, S., Christensen, F. E., Craig, W. W.,

Hailey, C., Harrison, F., Krivonos, R., ... Zhang, W. W. (2016). A Hard X-Ray Study of the Normal Star-Forming Galaxy M83 with NuSTAR. Astrophysical Journal, 824(2), [107]. https://doi.org/10.3847/0004-637X/824/2/107

\section{General rights}

Copyright and moral rights for the publications made accessible in the public portal are retained by the authors and/or other copyright owners and it is a condition of accessing publications that users recognise and abide by the legal requirements associated with these rights.

- Users may download and print one copy of any publication from the public portal for the purpose of private study or research.

- You may not further distribute the material or use it for any profit-making activity or commercial gain

- You may freely distribute the URL identifying the publication in the public portal 


\title{
A HARD X-RAY STUDY OF THE NORMAL STAR-FORMING GALAXY M83 WITH NuSTAR
}

\author{
M. Yukita ${ }^{1,2}$, A. E. Hornschemeier ${ }^{1,2}$, B. D. Lehmer ${ }^{1,2,3}$, A. Ptak ${ }^{1,2}$, D. R. WiK ${ }^{1,2}$, A. ZeZas ${ }^{4,5}$, V. Antoniou ${ }^{5}$, \\ T. J. Maccarone ${ }^{6}$, V. Replicon ${ }^{7}$, J. B. Tyler ${ }^{2,8}$, T. Venters $^{2}$, M. K. Argo ${ }^{9}$, K. Bechtol ${ }^{10}$, S. Boggs $^{11}$, F. E. Christensen ${ }^{12}$, \\ W. W. Craig $^{9,13}$, C. Hailey ${ }^{14}$, F. Harrison ${ }^{15}$, R. Krivonos ${ }^{11,16}$, K. Kuntz ${ }^{1}$, D. Stern ${ }^{17}$, And W. W. Zhang ${ }^{2}$ \\ ${ }^{1}$ The Johns Hopkins University, Homewood Campus, Baltimore, MD 21218, USA \\ ${ }^{2}$ NASA Goddard Space Flight Center, Code 662, Greenbelt, MD 20771, USA \\ ${ }^{3}$ Department of Physics, University of Arkansas, Fayetteville, AR 72701, USA \\ ${ }^{4}$ Physics Department, University of Crete, Heraklion, Greece \\ ${ }^{5}$ Harvard-Smithsonian Center for Astrophysics, 60 Garden Street, Cambridge, MA 02138, USA \\ ${ }^{6}$ Department of Physics, Texas Tech University, Lubbock, TX 79409, USA
${ }^{7}$ Department of Physics, New Mexico Institute of Mining and Technology, Socorro, NM 87801, USA \\ ${ }^{8}$ Institute for Astrophysics and Computational Sciences, Department of Physics, The Catholic University of America, Washington, DC 20064, USA \\ 9 Jodrell Bank Centre for Astrophysics, The University of Manchester, Oxford Road, Manchester M13 9PL, UK \\ ${ }^{10}$ Kavli Institute for Cosmological Physics, Chicago, IL 60637, USA \\ ${ }^{11}$ Space Science Lab, University of California, Berkeley, CA 94720, USA \\ ${ }^{12}$ National Space Institute, Technical University of Denmark, DK-2100 Copenhagen, Denmark \\ ${ }^{13}$ Lawrence Livermore National Laboratory, Livermore, CA, USA \\ ${ }^{14}$ Columbia University, New York, NY, USA \\ ${ }^{15}$ Caltech Division of Physics, Mathematics and Astronomy, Pasadena, CA, USA \\ ${ }^{16}$ Space Research Institute, Russian Academy of Sciences, Profsoyuznaya 84/32, 117997 Moscow, Russia \\ ${ }^{17}$ Jet Propulsion Laboratory, California Institute of Technology, Pasadena, CA 91109, USA \\ Received 2015 September 9; accepted 2016 March 25; published 2016 June 20
}

\begin{abstract}
We present the results from sensitive, multi-epoch NUSTAR observations of the late-type star-forming galaxy M83 $(d=4.6 \mathrm{Mpc})$. This is the first investigation to spatially resolve the hard $(E>10 \mathrm{keV}) \mathrm{X}$-ray emission of this galaxy. The nuclear region and $\sim 20$ off-nuclear point sources, including a previously discovered ultraluminous X-ray source, are detected in our NUSTAR observations. The X-ray hardnesses and luminosities of the majority of the point sources are consistent with hard X-ray sources resolved in the starburst galaxy NGC 253. We infer that the hard X-ray emission is most likely dominated by intermediate accretion state black hole binaries and neutron star low-mass X-ray binaries (Z-sources). We construct the X-ray binary luminosity function (XLF) in the NUSTAR band for an extragalactic environment for the first time. The M83 XLF has a steeper XLF than the X-ray binary XLF in NGC 253, which is consistent with previous measurements by Chandra at softer X-ray energies. The NuSTAR integrated galaxy spectrum of M83 drops quickly above $10 \mathrm{keV}$, which is also seen in the starburst galaxies NGC 253, NGC 3310, and NGC 3256. The NuSTAR observations constrain any active galactic nucleus (AGN) to be either highly obscured or to have an extremely low luminosity of $\lesssim 10^{38} \mathrm{erg} \mathrm{s}^{-1}(10-30 \mathrm{keV})$, implying that it is emitting at a very low Eddington ratio. An X-ray point source that is consistent with the location of the nuclear star cluster with an X-ray luminosity of a few times $10^{38} \mathrm{erg} \mathrm{s}^{-1}$ may be a low-luminosity AGN but is more consistent with being an X-ray binary.
\end{abstract}

Key words: galaxies: individual (M83) - galaxies: starburst - galaxies: star formation - X-rays: galaxies

\section{INTRODUCTION}

The NUSTAR observatory, the first focusing hard X-ray $(E>10 \mathrm{keV})$ telescope in orbit (Harrison et al. 2013), is capable of spatially resolving the components of nearby $(d<5 \mathrm{Mpc})$ star-forming galaxies in this energy range for the first time. Hard X-ray emission in star-forming galaxies lacking an active galactic nucleus (AGN) originates primarily from X-ray binaries: mainly high-mass X-ray binaries (HMXB) and ultraluminous X-ray sources (ULXs). There are other contributions, chiefly from low-mass X-ray binaries (LMXBs), but also possibly from accretion onto a supermassive black hole (SMBH) and perhaps diffuse non-thermal inverse Compton (IC) emission (Lehmer et al. 2013, 2015; Wik et al. 2014b; Ptak et al. 2015). However, the IC component results from the interaction of cosmic rays with IR and microwave background photons, and is likely to be negligible at moderately hard X-ray energies (e.g., see Wik et al. 2014b for the case of NGC 253).
We are conducting a NUSTAR starburst galaxy survey to investigate the hard X-ray properties of a sample of six nearby galaxies (NGC 253, Arp 299, M83, M82, NGC 3310, and NGC 3256) spanning star formation rates (SFRs) of 1-100 $M_{\odot} \mathrm{yr}^{-1}$. We are characterizing the $0.5-30 \mathrm{keV}$ spectral energy distribution (SED) of these galaxies (Lehmer et al. 2015; Ptak et al. 2015), which is of great cosmological importance. For example, this SED is used to perform important k-corrections for high- $z$ galaxies observed in the deepest Chandra X-ray surveys (Lehmer et al. 2016, submitted to the ApJ). For the closest galaxies ( $d<5 \mathrm{Mpc}$; NGC 253, M82, and M83), the very deep NUSTAR exposures allow us to conduct detailed studies of the spatially resolved point-source population.

In-depth studies of the starburst galaxy NGC 253 (Lehmer et al. 2013; Wik et al. 2014b) with NuSTAR and Chandra have demonstrated the utility of coordinated observations with these two facilities and hard X-ray color-color and color-intensity diagnostics to determine the states of $\mathrm{X}$-ray binaries in external galaxies. In NGC 253, the majority of the luminous X-ray 
binaries were found to have properties consistent with (stellarmass) black hole candidates in the intermediate state, which is an important measurement of the state of the population of $\mathrm{X}$-ray binaries in a star-forming environment. We now seek to answer the question of whether or not the preponderance of black holes in intermediate states is ubiquitous among starforming galaxies as well as to further investigate other populations, such as neutron star (NS) LMXBs, which might have similar colors.

It also appears that star-forming galaxies are dominated by ULXs and have overall spectra with steep turnovers at $E>10 \mathrm{keV}$ (e.g., NGC 253; Wik et al. 2014b; NGC 3310 and NGC 3256; Lehmer et al. 2015). This hard-energy turn over around $6-10 \mathrm{keV}$ is also seen for bright individual ULXs studied by XMM-Newton and NuSTAR (Stobbart et al. 2006; Gladstone et al. 2009; Walton et al. 2013, 2014; Bachetti et al. 2013; Rana et al. 2015) and is interpreted as super-Eddington accretion onto a stellar-mass black hole instead of an intermediate-mass black hole in a low/hard accretion state (e.g., Shakura \& Sunyaev 1973; King et al. 2001; Poutanen et al. 2007; Gladstone et al. 2009; Sutton et al. 2013).

Detailed investigation of the spectra of ULXs and other components contributing to the total galaxy spectrum requires observing very nearby galaxies. Even for galaxies only $<10$ Mpc away, X-ray binaries can be unresolved by NuSTAR's 58" half-power diameter point-spread function (PSF).

Therefore, we obtained a deep NuSTAR observation of the nearby star-forming galaxy M83, a face-on late-type (SABc) spiral galaxy. Its proximity $(d=4.61 \pm 0.20 \mathrm{Mpc}$; Saha et al. 2006) and large optical angular extent ${ }^{18}\left(D_{25}=12\right.$ !. $\times 11$ '.5, $17 \times 15 \mathrm{kpc})$ make it suitable for a point-source population study. We are able to achieve high efficiency in detecting point sources as compared to the edge-on galaxy NGC 253, whose resolved sources, owing to an overall more compact distribution, are more spatially blended with each other.

M83 has been studied quite intensively over a variety of wavelengths, including in the X-ray band. Chandra has observed this galaxy for $\sim 800 \mathrm{ks}$, identifying 398 point sources (Long et al. 2014, hereafter L14), including X-ray binaries down to $L_{\mathrm{X}}<10^{36} \mathrm{erg} \mathrm{s}^{-1}$ in the $0.35-8.0 \mathrm{keV}$ band. Notably, L14 found that the observed X-ray binary luminosity function (XLF) does not match the predicted luminosity function (LF) scaled from M83's SFR and stellar mass. The observed LF suggests that the substantial LMXB population dominates over the HMXBs associated with the current ongoing star-forming activity (i.e., Boissier et al. 2005). Ducci et al. (2013) investigated a number of bright point sources with $X M M$-Newton and found an overall X-ray binary LF that is consistent with the Chandra measurements. There are a couple of bright, individual sources in M83 that are also notable. Soria et al. (2012) discovered a ULX about $1^{\prime}$ to the east of the nucleus that is likely to have a red giant counterpart. There is another ULX candidate at the location of the edge of the stellar disk reported by Immler et al. (1999) and Stobbart et al. (2006).

So far, no AGN activity has been reported in M83. However, there is an X-ray point source identified in the Chandra observations as luminous as a few times $10^{38} \mathrm{erg} \mathrm{s}^{-1}$, at the location of a nuclear star cluster (Soria \& Wu 2003; Long et al. 2014). This source is a good candidate for the center of the

\footnotetext{
18 The galaxy's size is defined by the $B$-band surface brightness level of $25 \mathrm{mag}^{\operatorname{arcsec}^{-2}}$.
}

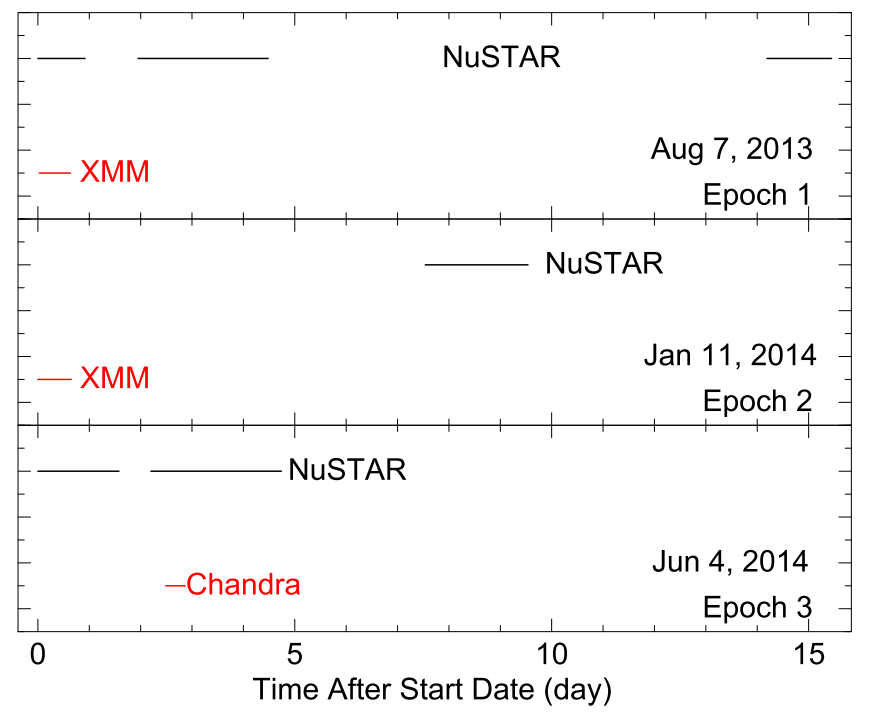

Figure 1. NUSTAR data on M83 were gathered in three separate observing campaigns. Shown are the relative NuSTAR, XMM-Newton, and/or Chandra observation times for each epoch. The start date for each epoch is indicated.

galaxy and a low-luminosity AGN, but the authors also suggest the possibility that it is an X-ray binary.

The goals of this paper are to identify the point sources detected by NUSTAR at hard energies (4-25 keV), to examine the point-source population by investigating luminosities and spectral colors, to characterize the broadband SED, and to constrain the nuclear activity in M83. We assume that the distance to M83 is $4.61 \mathrm{Mpc}$, for which $1^{\prime \prime}$ corresponds to 22 pc. Unless noted otherwise, the quoted uncertainties correspond to $90 \%$ confidence intervals for one interesting parameter.

\section{DATA AND DATA REDUCTION}

\subsection{NuSTAR Data}

NuSTAR observed M83 in 2013 and 2014 over three epochs as part of the NuSTAR Starburst Galaxy Survey. The three $N U S T A R$ epochs were simultaneous or nearly simultaneous with either XMM-Newton or Chandra observations, which constrain the lower-energy ( $E \lesssim 3 \mathrm{keV}$ ) emission and identify sources which may potentially be confused with NuSTAR's larger beam. We show these observational intervals graphically in Figure 1 and provide the observation log in Table 1.

We reduced the NUSTAR data using HEASOFT 6.15 and CALDB v. 20131223. Specifically, we processed the data using the nupipeline script, which creates calibrated and screened level 2 event lists from level 1 data. The resultant sum of the good time intervals is also listed in Table 1.

The optical extent of M83 $(12 ! 9 \times 11.5)$ is comparable to the field of view (FOV; Figure 2) of NuSTAR $\left(13^{\prime} \times 13^{\prime}\right)$; however, the center of the galaxy was placed a few arcminutes offset from the center of the FOV. This placement results in partial covering of M83 in each observation. Since the NuSTAR observations were taken with different roll angles, over the course of the three observations the entire $D_{25}$ region is covered after merging the data (see Figure 2).

We note that there are two bright objects, the Shapley supercluster and IC $4329 \mathrm{~A}$, located within a $1^{\circ}-5^{\circ}$ annulus centered on M83, which result in stray light (unfocused X-rays entering between the optics module and aperture stops) on the NUSTAR detectors. Unfortunately, the stray light pattern is 
Table 1

Observation Log

\begin{tabular}{|c|c|c|c|c|c|c|}
\hline \multicolumn{3}{|l|}{ NUSTAR } & \multicolumn{4}{|c|}{ XMM-Newton/Chandra } \\
\hline Date & ObsID & Net Exposure (ks) & Observatory & Date & ObsID & Net Exposure (ks) \\
\hline \multicolumn{7}{|c|}{ Epoch 1} \\
\hline 2013 Aug 7 & 50002043002 & 42 & XMM-Newton & 2013 Aug 7 & 0723450101 & 41 \\
\hline 2013 Aug 9 & 50002043004 & 80 & $\ldots$ & $\ldots$ & $\ldots$ & $\ldots$ \\
\hline 2013 Aug 21 & 50002043006 & 43 & $\ldots$ & $\cdots$ & $\cdots$ & $\cdots$ \\
\hline \multicolumn{7}{|c|}{ Epoch 2} \\
\hline 2014 Jan 19 & 50002043008 & 81 & XMM-Newton & 2014 Jan 11 & 0723450201 & 20 \\
\hline \multicolumn{7}{|c|}{ Epoch 3} \\
\hline 2014 Jun 4 & 50002043010 & 70 & $\ldots$ & $\ldots$ & $\ldots$ & \\
\hline 2014 Jun 7 & 50002043012 & 110 & Chandra & 2014 Jun 7 & 16024 & 30 \\
\hline
\end{tabular}

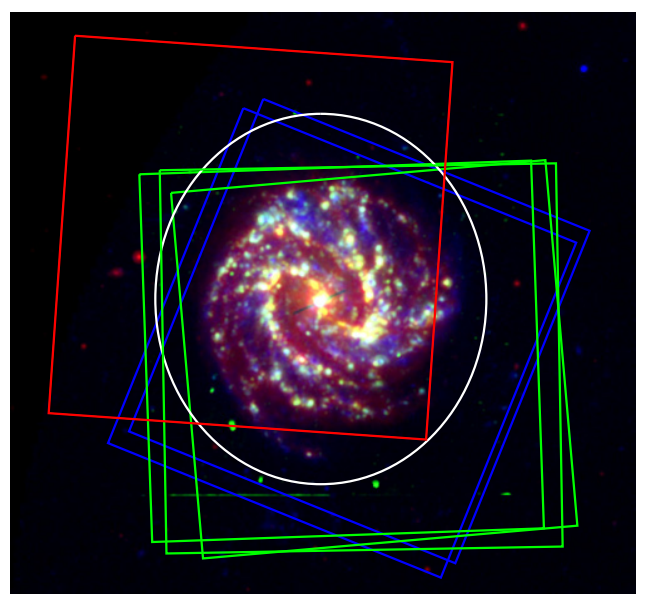

Figure 2. GALEX FUV(blue), continuum-subtracted $\mathrm{H} \alpha$ (green), and Spitzer $24 \mu \mathrm{m}$ (red) images of M83 (from the Spitzer Local Volume Survey, Dale et al. 2009). North is up and east to the left. White ellipse indicates the optical extent $\left(D_{25}\right)$ of the galaxy. Boxes represent the NuSTAR FOV $\left(13^{\prime} \times 13^{\prime}\right)$; green for 2013 August, red for 2014 January, and blue for 2014 June observations.

apparent in all of the observations. However, stray light can be estimated using the location of those bright sources and the observed roll angle. We used the nustar_stray_light ${ }^{19}$ procedure to estimate the affected regions on each detector for each observation. IC 4239A contaminated the small region to the east of M83 in both the FPMA and FPMB data. The Shapley supercluster only appeared on one of either FPMA or FPMB (i.e., one of the telescopes), but the stray light is extended over a larger area. The contamination from both IC 4329A and the Shapley supercluster covered more than half of the total area of either the FPMA or FPMB detector. We discarded the data which suffered from the Shapley supercluster stray light. Specifically, we only utilize FPMA for the January 2014 observation and FPMB for the remaining five observations. We also eliminated the region affected by IC 4239A in these data. As a result, we partially missed the southeast region of $D_{25}$ in our analysis. In Figure 3, we show the co-added NuSTAR exposure map after removing the contaminated areas, which presents the area coverage with effective exposures of our M83 observations.

\footnotetext{
19 https://github.com/bwgref/nustar_stray_light
}

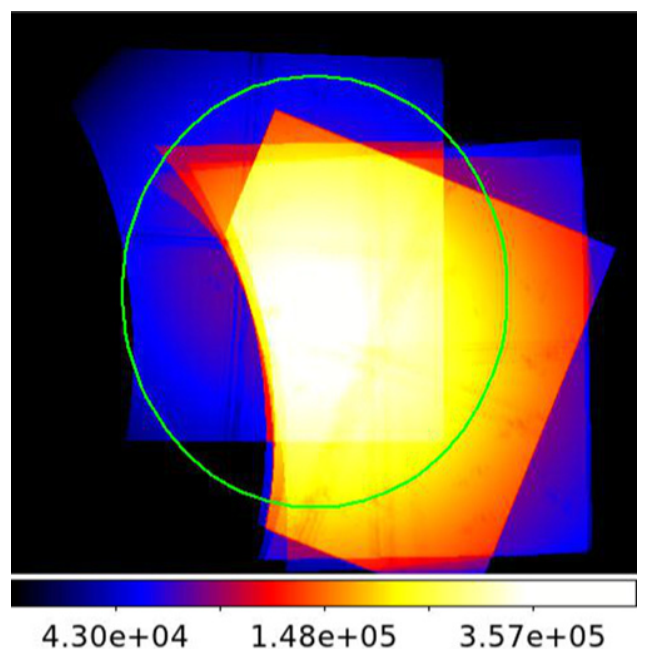

Figure 3. Exposure map of the co-added NUSTAR data presents the effective exposure time for different locations of the galaxy. (North is up and east to the left.) Green ellipse indicates $D_{25}$. The pixel values are in units of seconds.

\subsection{Simultaneous XMM-Newton and Chandra Observations}

XMM-Newton observations took place on 2013 August 8 (epoch 1) and 2014 January 11 (epoch 2) as part of an AO 12 GO program to monitor the newly discovered ULX CXO 133705-295207 (P.I. Kuntz). The detailed XMM-Newton analysis of the ULX is presented in Soria et al. (2015). Our primary use for the XMM-Newton data is to locate bright point sources and to characterize the $0.5-8.0 \mathrm{keV}$ spectral shape of the galaxy and bright sources. Because the XMM-Newton PN detector alone achieves $4-10 \mathrm{keV}$ sensitivity, which is similar to our NuSTAR 4-10 keV sensitivity, we utilized the PN data only to avoid complication of data analysis between the different XMM-Newton detectors. Our choice of the PN detector over MOS was motivated by the better signal-to-noise ratio $(\mathrm{S} / \mathrm{N})$ and larger FOV (i.e., due to the loss of two CCD chips of MOS 1). The XMM-Newton data were reduced using SAS v.13.0.0. The level 1 data were reprocessed using the SAS scripts, epchain, and then pn-filter is applied to eliminate high background periods. The resulting exposures are tabulated in Table 1.

Chandra observed M83 on 2014 June 2 (epoch 3) using the Advanced CCD Imaging Spectrometer (ACIS) as a part of the Chandra campaign of the NuSTAR starburst galaxy survey (P.I. 

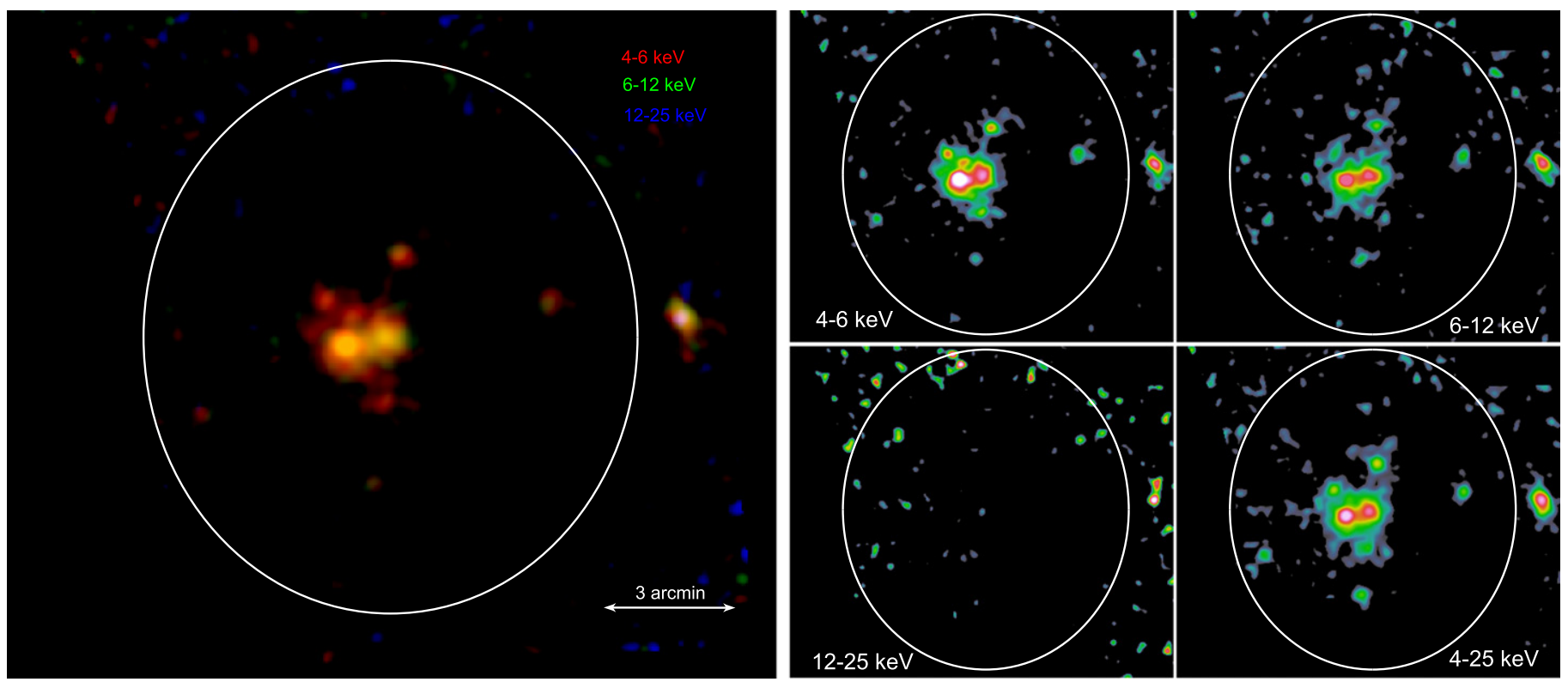

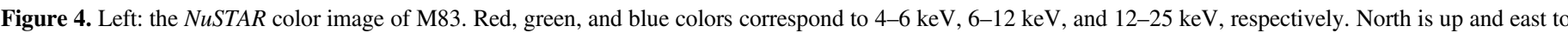

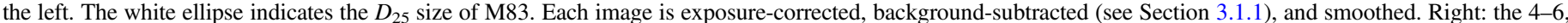

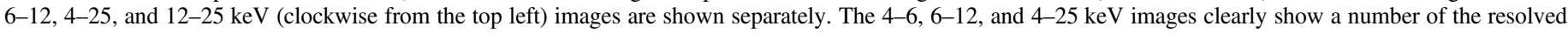
point sources in M83.

Hornschemeier). To capture the entire $D_{25}$, we used the ACIS-I array, which has a wide FOV $\left(16^{\prime} \times 16^{\prime}\right)$ that complements well the NUSTAR FOV. The Chandra data reduction and analysis were performed with $C I A O$ version 4.6 and CALDB 4.6.3. Chandra data were reprocessed using the CIAO tool chandra_repro, which applies time-dependent gain and charge transfer inefficiency corrections and removes bad pixels and bad grade events. We removed high background periods applying the sigma-clipping method. Namely, we used the $C I A O$ deflare script, rejecting the intervals that exceeded $3 \sigma$ above the mean. The final net exposure was $29.6 \mathrm{ks}$ of the original $30 \mathrm{ks}$ observation. Figure 1 illustrates the relative NuSTAR, XMM-Newton, and Chandra coverages for each epoch.

Both the XMM-Newton and Chandra observations were spatially registered to the Chandra point source catalog of L14.

\section{SPATIAL ANALYSIS}

The NUSTAR M83 observations spatially resolve multiple point-like X-ray sources including the known ULX source (see Figure 4). To maximize the $\mathrm{S} / \mathrm{N}$, we co-added observations from all three epochs, which results in an effective exposure of $\sim 370 \mathrm{ks}$ in the central region of M83 (see Figure 3). The first and third epoch observations were aligned using the position of CXO 133705-295207 (x299 in L14), which was obtained by fitting a circular Gaussian to $4-10 \mathrm{keV}$ images of the ULX. In general, using multiple point sources for alignment is preferred, but the $\mathrm{S} / \mathrm{N}$ of the majority of the point sources for each observation was low. Nevertheless, since x299 was not bright in the second epoch observation, we used other bright sources to align the data to the remaining observations. All of the aligned event lists were merged using the FTOOLS ftmerge utility to create a combined, deep exposure event list. The final event list was registered using the position of the ULX to the published Chandra coordinates of $\mathrm{x} 299$ by L14. In our astrometric solution, we assume no rotation since there is not a sufficient number of sources to constrain it.

The left panel of Figure 4 shows the NuSTAR false color image of M83 for the merged data. The individual 4-6, 6-12, $12-25$, and $4-25 \mathrm{keV}$ images are displayed in the right panel of Figure 4. All of the images are exposure-corrected, background-subtracted, and smoothed. The 4-6, 6-12, and 4-25 keV images clearly show that a number of point sources are resolved within the optical extent of M83. These resolved sources are not obvious in the $12-25 \mathrm{keV}$ band image. In the next subsection, we identify the resolved point sources and measure their fluxes in the NuSTAR images.

\subsection{Point-source Identification}

NUSTAR fluxes for point sources were obtained via pointspread function (PSF) fitting, as opposed to aperture photometry, because some sources contain flux from broad PSF wings underneath them. We used Sherpa (included as a part of $C I A O$ ) for spatial fitting of the NUSTAR images. In this section, we describe the method used to identify and measure the NuSTAR fluxes of 21 resolved point sources. The outline of the procedure is to (1) create the XMM-Newton/Chandra pointsource catalog, (2) measure NuSTAR fluxes based on the XMMNewton/Chandra source positions, and (3) reject faint sources whose fluxes are below $90 \%$ confidence levels.

\subsubsection{NuSTAR Background Modeling}

For photometry, the NUSTAR background must also be taken into account. The NUSTAR background consists of the focused cosmic X-ray background, stray light from the cosmic X-ray background (referred as the aperture background), instrument background, and reflected solar X-rays (Wik et al. 2014a). The spatial variations of the background surface brightness are significant, and therefore we use the nuskybgd script (Wik et al. 2014a) to create model background images for each observation. The nuskybgd script characterizes the spectral 
and spatial parameters of the observed background in the source-free region of the observations and then extrapolates across the FOV (see Wik et al. 2014a, for details). In Sherpa, the simulated background images are fit to a two-dimensional (2D) polynomial function to analytically determine the spatial distribution of the background model. We also note that an onaxis PSF model (An et al. 2014; Madsen et al. 2015) is applied even to off-axis sources. This approach may therefore introduce systematic uncertainties due to possibly oversimplified background and PSF models.

To estimate systematic errors, we simulate a background image by adding Poisson noise onto a nuskybgd background model image and then added simulated point sources at arbitrary locations. We compared the input and measured simulated point source fluxes and concluded that using an analytic background model and an on-axis PSF model introduces $<10 \%$ systematic uncertainties for sources within $5^{\prime}$ from the optical axis. All of our point sources are detected within $5^{\prime}$ from the optical axis, and statistical errors are typically much larger than $10 \%$. Therefore, the systematic error is negligible.

We also validate our method by comparing it to the tool developed by Wik et al. (2014b), which uses both the simulated background images and customized PSF models for individual sources. We applied the Wik et al. (2014b) method to one of our three epochs and confirmed that flux measurements performed with both methods agree with each other within $90 \%$ confidence.

\subsubsection{Determining the NuSTAR Point Source List}

Since our NuSTAR observations are taken simultaneously or nearly simultaneously with either XMM-Newton or Chandra, candidate point-source detection is performed using these higher spatial resolution images to mitigate source confusion. We point out that the Chandra and XMM-Newton exposure times were sufficiently long to ensure that the Chandra and XMM-Newton observation sensitivities in the $4-8 \mathrm{keV}$ band exceed the sensitivity of our NUSTAR observations in the same band. We ran the SAS source detection tool detect_chain with 16 spline nodes and an upper limit likelihood of $\overline{15}$ on the XMM-Newton PN images in the $4-10 \mathrm{keV}$ band, which were chosen to maximize the energy range which overlaps with NuSTAR. For the Chandra data, we ran wavdetect with scale parameters of $1.0,1.4,2.0,2.25,4.0$, and 5.56 on the 4-8 keV image, since the Chandra effective area drops off rapidly above $7 \mathrm{keV}$. Then, we merged the three point-source lists from each epoch to create a master point-source catalog.

We note that the nuclear region is detected as one source in the XMM-Newton observations, whereas it is clearly resolved into at least seven sources in the Chandra 4-8 keV image. Our final Chandra/XMM-Newton source list contains 39 sources, 38 of which were reported in L14. One source that was not included in L14 is located outside of the FOV of their Chandra observations.

We characterized the NuSTAR point sources that were detected at or above the $90 \%$ confidence level in the co-added NUSTAR 4-10 keV image via PSF fitting. In our PSF-fitting procedure, NUSTAR point sources were modeled with 2D Gaussians with widths of 5"; these Gaussians were convolved with the PSFs to model the sources. We chose to use a 2D Gaussian instead of a delta function to take into account the uncertainties in the PSF shape. We note that the model background parameters constrained from the nuskybgd images were fixed during the fitting.

Using the XMM-Newton/Chandra point-source position in the master catalog, we initially fit the brightest point source to the NuSTAR 4-10 keV image. The source positions were derived parameters, and so offsets, distortions, and rotations of the images were allowed in the fit. Once we obtained the best-fit parameters for a source, we added the next brightest source in the XMM-Newton/Chandra catalog to the model, and thereafter continued source by source, moving down in count rate. When the resulting source flux was negative and/or the best-fit position was more than $12^{\prime \prime}$ (5 pixel) away from the original point-source position in the master catalog, the source was considered to be undetected. We also discarded those sources whose fluxes were not significant at the $90 \%$ confidence level. Having identified which Chandra or XMMNewton point sources are detected significantly in the 4-10 keV NuSTAR image, we used their positions (fixed during the PSF fitting) to measure their 4-6, 6-12, 12-25, and 4-25 keV fluxes while fixing the source positions during PSF fitting. We note that in our method, we should be able to identify and measure sources with fluxes above $\sim 2.3 \times 10^{-4} \mathrm{ct} \mathrm{s}^{-1}, \sim 0.80 \times 10^{-4} \mathrm{ct}$ $\mathrm{s}^{-1}, \sim 1.1 \times 10^{-4} \mathrm{ct} \mathrm{s}^{-1}, \sim 1.1 \times 10^{-4} \mathrm{ct} \mathrm{s}^{-1}$, and $\sim 1.5 \times 10^{-4}$ ct $\mathrm{s}^{-1}$ in 4-10, 4-6, 6-12, 12-25, and 4-25 keV, respectively, for a $350 \mathrm{ks}$ exposure. However, these values may fluctuate depending on source positions due to background variations and neighboring sources.

We point out that we may have missed very obscured sources that do not appear in the XMM-Newton and/or Chandra images. However, we note that no very bright sources appeared in the NUSTAR $12-25 \mathrm{keV}$ image that were not also detected in the Chandra/XMM-Newton observations (see Figure 6).

There were a total of 21 NUSTAR sources with flux measurements. The results are listed in Table 2 along with the source ID of L14. We also calculate and list position differences between the NUSTAR and Chandra L14 positions. We note that some of the offsets listed in the table are large $\left(6^{\prime \prime}-10^{\prime \prime}\right)$. This may be due to rotation and PSF uncertainties that could not completely be taken into account using a 2D Gaussian model. We have compared the NuSTAR 4-10 keV images with the concurrent XMM-Newton and Chandra images in the same band. It would be less likely that sources detected in the XMM-Newton or Chandra images decreased in flux in the NuSTAR observations at the same energy band and different $X M M-N e w t o n / C h a n d r a$ undetected sources increased in flux to be above the detection threshold within a few arcseconds. However, we cannot exclude the possibility that a measured NUSTAR flux may not be truly associated with the identified Chandra or XMM-Newton source.

Figure 5 illustrates the location of the NuSTAR-detected point sources with Chandra source IDs (from L14). Overall, about half of the Chandra/XMM-Newton sources were detected in the NUSTAR observations, while sources often were not detected due to source confusion. For example, two NUSTAR sources are identified in the nuclear region with our PSF-fitting approach, whereas Chandra clearly resolved the region into seven point sources (see Figure 8). Similarly, there are several sources detected in the XMM-Newton and Chandra images near source $\mathrm{x} 248$ that are detected as a single source by NuSTAR. 
Table 2

M83 NuSTAR Point Sources

\begin{tabular}{|c|c|c|c|c|c|c|c|c|}
\hline \multirow[b]{2}{*}{ R.A. ${ }^{a}$} & & \multirow{2}{*}{$\begin{array}{c}\text { L14-NuSTAR } \\
\text { Offset } \\
(\operatorname{arcsec})\end{array}$} & \multirow{2}{*}{$\begin{array}{c}\text { Effective } \\
\text { Exp } \\
(\mathrm{ks})\end{array}$} & \multirow[b]{2}{*}{$\begin{array}{l}\mathrm{ID}^{\mathrm{c}} \\
\mathrm{L} 14\end{array}$} & \multicolumn{4}{|c|}{ Count Rates } \\
\hline & 0) decl. ${ }^{a}$ & & & & $(4-6 \mathrm{keV})$ & $(6-12 \mathrm{keV})$ & $\begin{array}{l}(12-25 \mathrm{keV}) \\
\left.\mathrm{s}^{-1}\right)\end{array}$ & $(4-25 \mathrm{keV})$ \\
\hline $13: 37: 04$ & $-29: 49: 27$ & 3.1 & 322.1 & $\mathrm{x} 281$ & $0.41_{-0.11}^{+0.12}$ & $0.54_{-0.14}^{+0.15}$ & $<0.11$ & $1.07_{-0.22}^{+0.22}$ \\
\hline $13: 36: 43$ & $-29: 51: 01$ & 6.3 & 221.1 & x029 & $1.09_{-0.17}^{+0.18}$ & $1.10_{-0.19}^{+0.20}$ & $<0.11$ & $2.22_{-0.29}^{+0.30}$ \\
\hline $13: 36: 49$ & $-29: 52: 30$ & 0.3 & 309.9 & x048 & $0.15_{-0.09}^{+0.10}$ & $0.21_{-0.10}^{+0.11}$ & $<0.13$ & $0.47_{-0.17}^{+0.19}$ \\
\hline $13: 36: 52$ & $-29: 53: 34$ & 0.5 & 305.2 & x073 & $0.17_{-0.09}^{+0.10}$ & $0.22_{-0.11}^{+0.12}$ & $<0.08$ & $0.45_{-0.17}^{+0.18}$ \\
\hline $13: 36: 57$ & $-29: 49: 11$ & 1.3 & 318.2 & x145 & $0.46_{-0.12}^{+0.13}$ & $0.57_{-0.14}^{+0.15}$ & $<0.20$ & $1.21_{-0.22}^{+0.23}$ \\
\hline $13: 36: 57$ & $-29: 50: 58$ & 6.7 & 361.5 & x165 & $0.34_{-0.11}^{+0.12}$ & $0.51_{-0.13}^{+0.14}$ & $<0.15$ & $0.96_{-0.21}^{+0.22}$ \\
\hline $13: 36: 57$ & $-29: 53: 38$ & 1.8 & 345.1 & x152 & $0.55_{-0.12}^{+0.12}$ & $0.58_{-0.13}^{+0.14}$ & $<0.05$ & $1.13_{-0.20}^{+0.21}$ \\
\hline $13: 36: 58$ & $-29: 47: 25$ & 3.9 & 255.1 & x155 & $0.17_{-0.12}^{+0.13}$ & $0.36_{-0.16}^{+0.17}$ & $<0.22$ & $0.73_{-0.25}^{+0.27}$ \\
\hline $13: 36: 59$ & $-29: 50: 01$ & 1.5 & 342.9 & x185 & $1.43_{-0.16}^{+0.16}$ & $1.18_{-0.16}^{+0.17}$ & $0.17_{-0.11}^{+0.12}$ & $2.86_{-0.26}^{+0.26}$ \\
\hline $13: 37: 00$ & $-29: 52: 02$ & 3.3 & 349.8 & x193 & $1.08_{-0.25}^{+0.26}$ & $1.20_{-0.27}^{+0.27}$ & $<0.13$ & $2.25_{-0.40}^{+0.41}$ \\
\hline $13: 37: 01$ & $-29: 47: 46$ & 3.1 & 264.0 & $\mathrm{x} 252$ & $0.32_{-0.12}^{+0.13}$ & $0.52_{-0.16}^{+0.18}$ & $<0.15$ & $1.11_{-0.25}^{+0.27}$ \\
\hline $13: 37: 01$ & $-29: 51: 31$ & 10.9 & 305.2 & x246 & $0.93_{-0.26}^{+0.27}$ & $0.46_{-0.25}^{+0.27}$ & $<0.17$ & $1.48_{-0.40}^{+0.41}$ \\
\hline $13: 37: 01$ & $-29: 51: 56$ & 0.3 & 343.9 & $\mathrm{x} 233$ & $1.93_{-0.34}^{+0.36}$ & $2.25_{-0.35}^{+0.37}$ & $0.33_{-0.17}^{+0.19}$ & $4.50_{-0.53}^{+0.54}$ \\
\hline 13:37:01 & $-29: 53: 25$ & 2.0 & 349.3 & x248 & $1.29_{-0.16}^{+0.16}$ & $0.66_{-0.14}^{+0.15}$ & $0.23_{-0.11}^{+0.12}$ & $2.28_{-0.25}^{+0.25}$ \\
\hline $13: 37: 02$ & $-29: 55: 19$ & 1.5 & 292.3 & $\mathrm{x} 258$ & $0.83_{-0.14}^{+0.15}$ & $1.27_{-0.18}^{+0.19}$ & $0.27_{-0.13}^{+0.14}$ & $2.46_{-0.27}^{+0.28}$ \\
\hline $13: 37: 04$ & $-29: 51: 19$ & 2.7 & 335.7 & x286 & $0.45_{-0.18}^{+0.18}$ & $0.10_{-0.16}^{+0.17}$ & $<0.16$ & $0.63_{-0.27}^{+0.28}$ \\
\hline $13: 37: 05$ & $-29: 52: 07$ & $0.0^{\mathrm{d}}$ & 353.0 & x299 & $4.85_{-0.26}^{+0.27}$ & $3.59_{-0.24}^{+0.25}$ & $0.20_{-0.11}^{+0.12}$ & $8.71_{-0.38}^{+0.38}$ \\
\hline $13: 37: 05$ & $-29: 53: 59$ & 4.8 & 345.5 & x284 & $0.43_{-0.11}^{+0.12}$ & $0.31_{-0.12}^{+0.13}$ & $0.14_{-0.11}^{+0.12}$ & $0.92_{-0.20}^{+0.21}$ \\
\hline $13: 37: 07$ & $-29: 51: 01$ & 0.5 & 346.2 & x321 & $1.32_{-0.16}^{+0.17}$ & $0.90_{-0.16}^{+0.17}$ & $<0.17$ & $2.38_{-0.25}^{+0.26}$ \\
\hline $13: 37: 13$ & $-29: 52: 01$ & 6.2 & 56.5 & x366 & $0.45_{-0.23}^{+0.28}$ & $0.56_{-0.27}^{+0.32}$ & $<0.15$ & $0.73_{-0.42}^{+0.48}$ \\
\hline $13: 37: 20$ & $-29: 53: 43$ & 6.1 & 49.7 & x403 & $0.89_{-0.28}^{+0.33}$ & $0.46_{-0.29}^{+0.34}$ & $0.30_{-0.26}^{+0.32}$ & $1.03_{-0.46}^{+0.51}$ \\
\hline
\end{tabular}

Notes.

a $N U S T A R$ source position.

b Offset between NUSTAR source position and the Chandra catalog by L14.

c Source ID by L14.

d Astrometry is registered to this source.

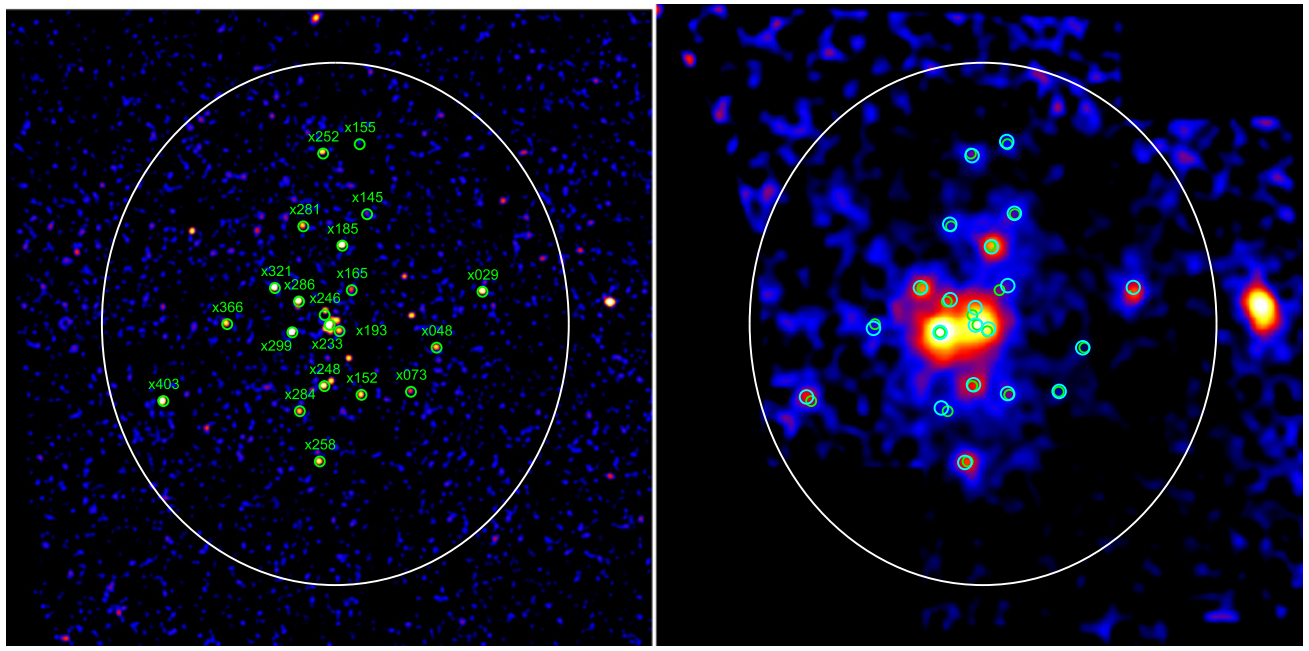

Figure 5. Left: The Chandra 4-8 keV image taken in 2014 June. North is up and east to the left. The white ellipse indicates the optical extent $\left(D_{25}\right)$ of the galaxy. Green circles indicate the Chandra positions of the point sources with NuSTAR flux measurements. The labels for each point source are the source IDs from L14. The Chandra image is smoothed with a Gaussian of width 1". 5. Right: the NuSTAR co-added 4-25 keV image. Cyan circles are the NuSTAR positions of the point sources. Green circles are the Chandra source positions. We note that only 21 out of 39 Chandra sources have NuSTAR flux measurements. Since bright sources are white in the Chandra image, it is clear that sources without NuSTAR fluxes are fainter. The NuSTAR image is smoothed with a Gaussian of 7 !" 5 width. The bright source located outside the galaxy $D_{25}$ in the west has $\log \left(L_{\mathrm{X}} / L_{\mathrm{opt}}\right)=-1$ (Ducci et al. 2013), which is a typical value for background AGNs (see Tzanavaris et al. 2006).

Figure 7 shows the correlation between the active starforming regions and the location of the NuSTAR point sources. About $25 \%$ of the sources are located in intense star-forming regions; however, there is no strong relation between the X-ray source locations and the spiral arms.
Instead of simply using the net exposure, the source count rates were obtained using the "effective exposures", listed in Table 2, which include corrections based on effective area at the detector locations, vignetting, and removal of detector area affected by stray light. To study the long-term X-ray variability 


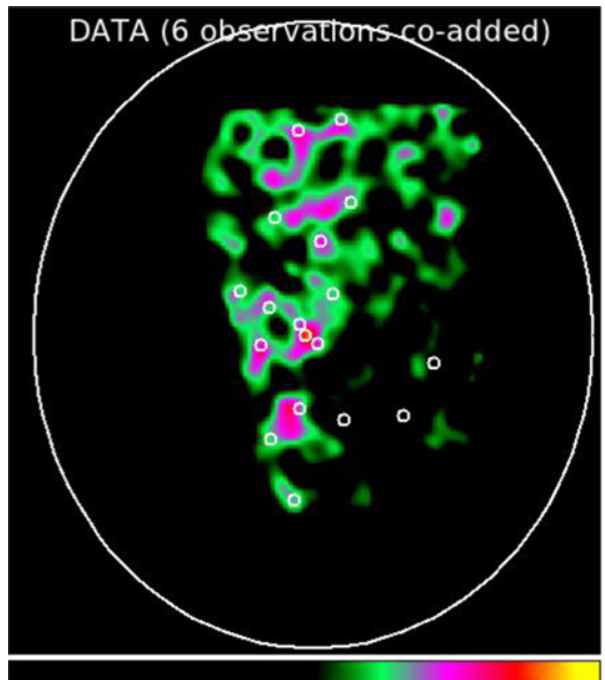

$\begin{array}{lllll}0.085 & 0.26 & 0.43 & 0.59 & 0.77\end{array}$

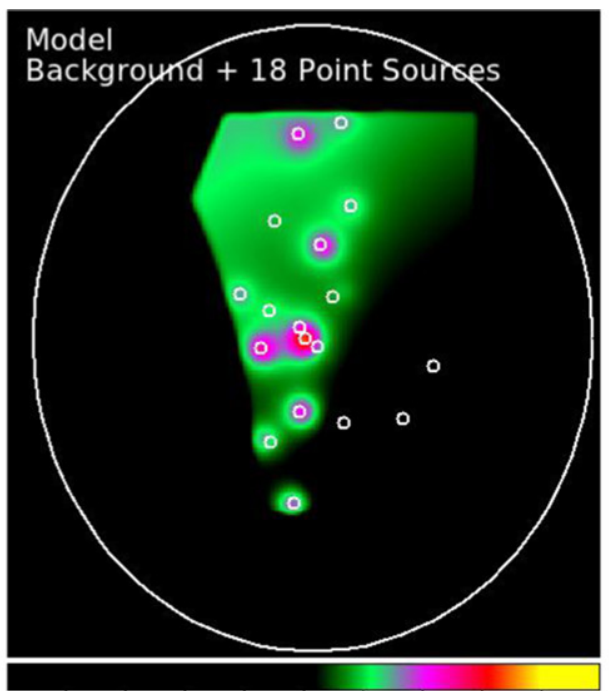

0.085

0.26

0.43

0.59

0.77

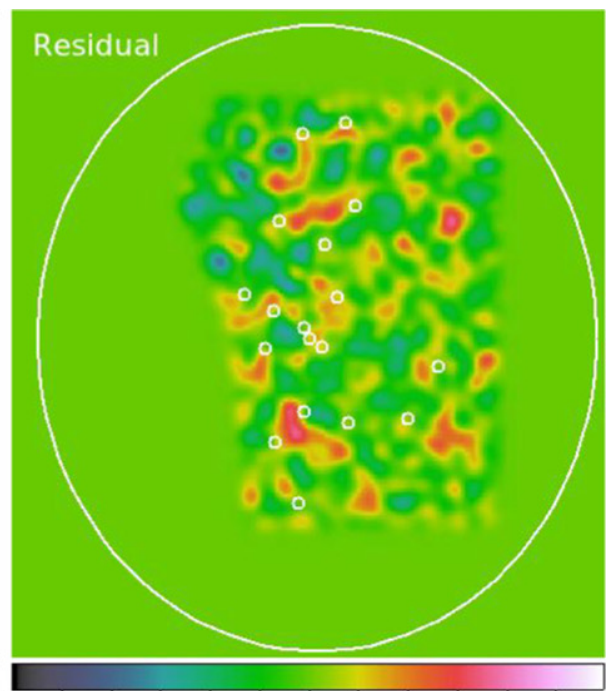

$\begin{array}{lllll}-0.2 & -0.1 & 0 & 0.1 & 0.2\end{array}$

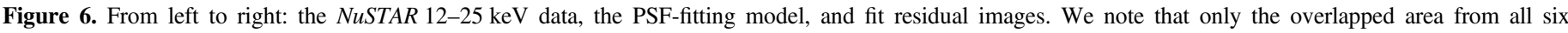

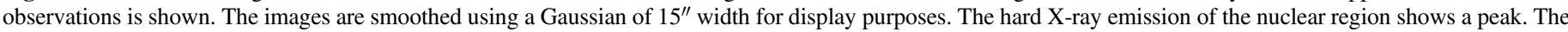

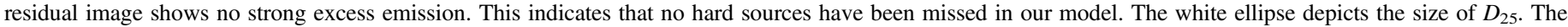

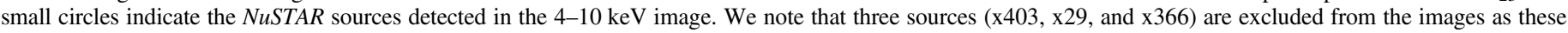
sources were missed in one of the three epochs.

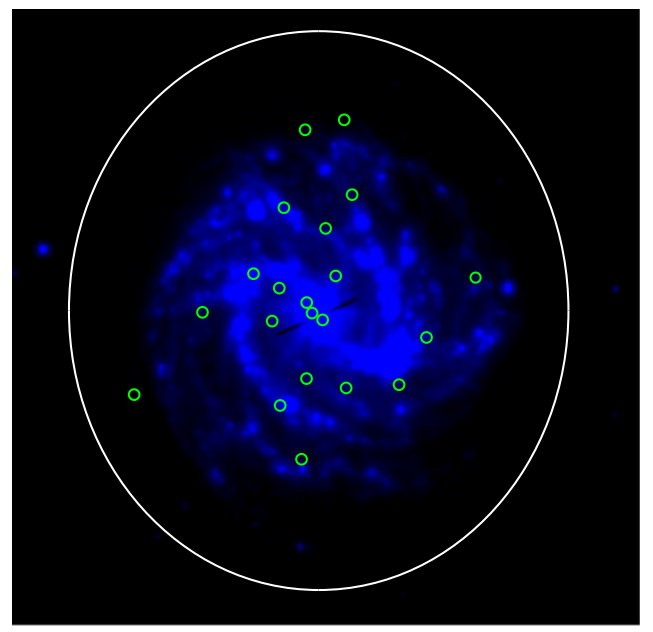

Figure 7. NUSTAR point sources overlaid on the GALEX FUV image. About $25 \%$ of the NUSTAR sources are located in active star-forming regions. North is up and east to the left. The white ellipse depicts the $D_{25}$ size.

in the 4-25 keV band probed by NuSTAR (see Section 3.3), we also measured the NuSTAR fluxes at each epoch. The measured fluxes are listed in Table 3. To validate our NuSTAR flux measurements, we compared the measured NuSTAR count rates to the Chandra/XMM-Newton count rates in the $4-6 \mathrm{keV}$ energy band for the same sources (see Figure 9) and found reasonable agreement.

\subsection{NuSTAR Color Diagnostics for Compact Binaries}

Differentiating BH binaries from NS LMXBs can be done by investigating the curvature in their spectra, disk temperature, and inner radius as we have shown for the bright M31 sources with very high $\mathrm{S} / \mathrm{N}$ ratio spectra when extending up to $20 \mathrm{keV}$ (Maccarone et al. 2016, MNRAS, submitted). Similarly, as black hole binaries move through different accretion states, their X-ray spectra change dramatically at $E>10 \mathrm{keV}$, much more so than at lower energies (e.g., Tananbaum et al. 1972; Done et al. 2007). NuSTAR's great increase in sensitivity at $>10 \mathrm{keV}$ over past missions has provided us with the opportunity to constrain the nature of the resolved sources and to investigate the accretion states of black hole binary populations in nearby galaxies for the first time (see also the NGC 253 study of Wik et al. 2014b). The color-intensity and color-color diagrams are powerful tools for diagnosing point sources which are too faint for spectral analysis. In this section, we construct $N u S T A R$ color-intensity and color-color diagrams to examine both the identities and accretion states of the X-ray point sources in M83.

Similar to Wik et al. (2014b), we compute the hardness ratios of the point sources using the NuSTAR count rates we obtained in the previous subsection. The hardness ratios are defined as follows. The NuSTAR hard color is $(\mathrm{H}-\mathrm{M}) /(\mathrm{H}+\mathrm{M})$, where $\mathrm{H}$ is the hard-band $(12-25 \mathrm{keV})$ count rate and $\mathrm{M}$ is the medium-band count rate $(6-12 \mathrm{keV})$. The $N u S T A R$ soft color is $(\mathrm{M}-\mathrm{S}) /(\mathrm{M}+\mathrm{S})$, where $\mathrm{S}$ is the soft band $(4-6 \mathrm{keV})$. These colors and intensities can be compared with well-studied Galactic BH binaries in different accretion states. Using high $\mathrm{S} / \mathrm{N}$ hard X-ray spectra of Galactic $\mathrm{BH}$ binaries from $R X T E$ observations, NuSTAR colors and intensities were calculated for seven Galactic BH binaries over the full range of accretion states, using the NUSTAR response. Intensities were computed assuming a distance of $4 \mathrm{Mpc}$. Similarly, a sample of 8 pulsar binaries were included in the same fashion (see A. Zezas et al. 2016, in preparation for more details).

The simulated colors and intensities for the Galactic $\mathrm{BH}$ binaries are shown for different accretion states. Different color symbols indicate different $\mathrm{BH}$ accretion states in Figures 10 and 11. Specifically, soft-, intermediate-, and hard-accretion states correspond to red, green, and blue points, respectively. Galactic accreting pulsars are plotted with magenta open squares. We also plot Galactic NS LMXBs using spectral fits from Church et al. (2012). Although high $\mathrm{S} / \mathrm{N}$ ratio spectra analysis distinguishes $\mathrm{BH}$ binaries from NS, NS LMXBs 

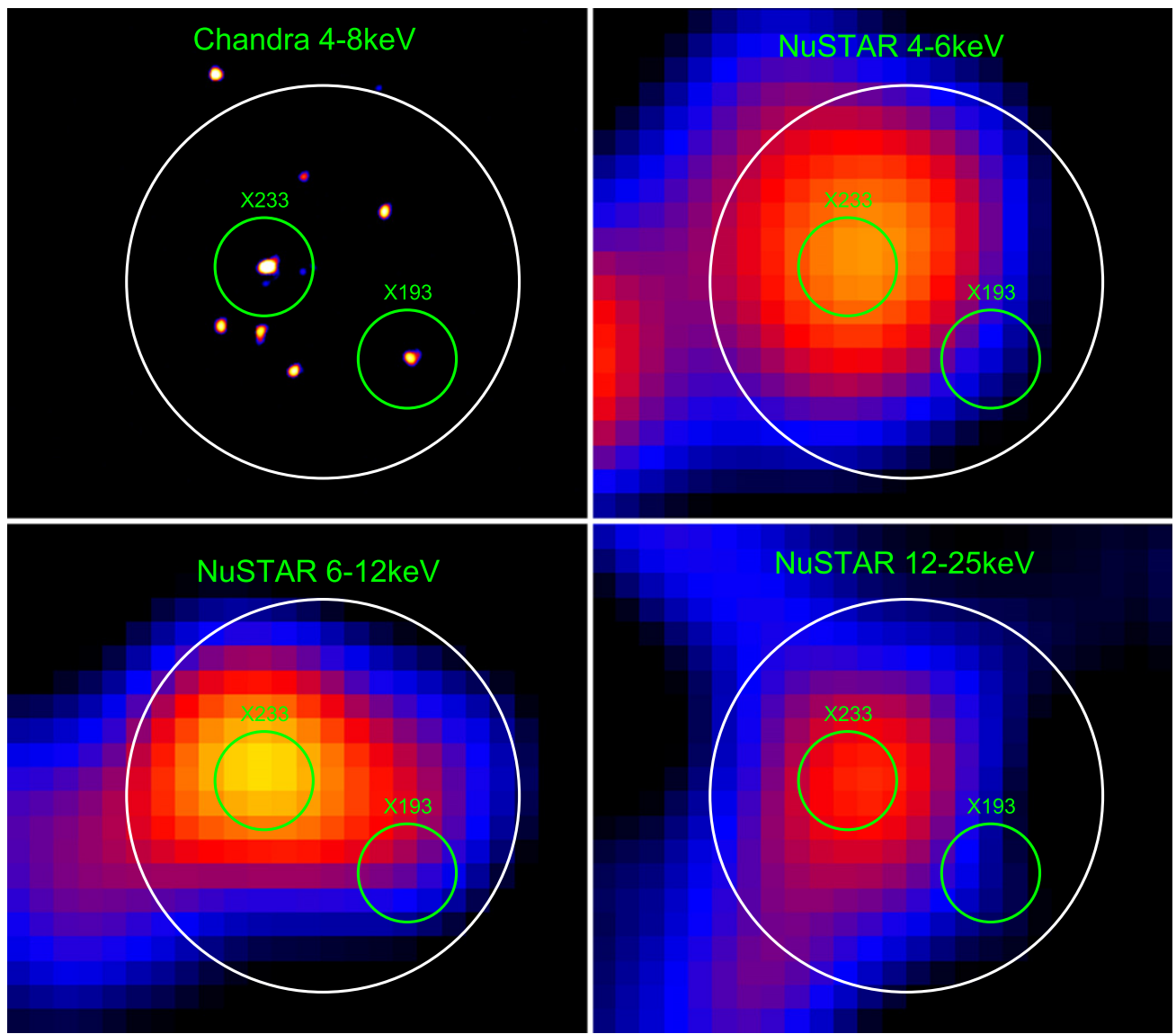

Figure 8. NUSTAR images of the central region of M83. North is up and east to the left. Source $\mathrm{x} 233$ is located at the position of the optical nucleus (nuclear star cluster). There are $\sim 7 \mathrm{X}$-ray sources resolved in the Chandra image. The white circle indicates a $20^{\prime \prime}$ radius aperture used for extracting the nuclear spectrum in Section 4.2. Green circles indicate the sources with NuSTAR flux measurements.

possess $N u S T A R$ colors and intensities which are similar to the Galactic BH binaries in our color diagnostic diagrams. The NS LMXBs in the plots are all "Z-sources"; another subgroup of NS LMXBs, atoll sources, are relatively faint in general and located below our M83 observation detection limit in the diagrams. We note, however, that the errors on our X-ray colors are large near the detection limit, and higher-quality data would be required to clearly differentiate between a highluminosity atoll source and a Z-source.

In addition to the Galactic BH binaries, accreting pulsars, and "Z-sources," several ULXs observed by NuSTAR (Bachetti et al. 2013; Walton et al. 2013, 2014; Rana et al. 2015) are included as gray upside-down triangles. We note that the ULXs are well-separated from the Galactic binary tracks in the colorintensity plot (Figure 10), as expected given their high luminosities. The Galactic NS LMXBs overlap with Galactic $\mathrm{BH}$ binaries in the intermediate-accretion state in the colorintensity plot. However, the two types may be differentiated in the color-color diagram, since NS binaries are likely to be softer in the NuSTAR hard band (12-25 keV). We plot the M83 NUSTAR sources with filled black diamonds and have annotated their L14 IDs. Fourteen out of the $21 \mathrm{NuSTAR}$ sources have upper limits on the hard-band flux. Therefore, in Figure 11, we plot only the seven sources with hard NuSTAR detections.

The brightest source in Figure 10 is $\mathrm{x} 299$, which appears to have colors similar to the other NUSTAR ULXs. The second brightest is $\mathrm{x} 233$, which is in the nuclear region, and may be confused with neighboring faint sources, making interpretation of this source difficult (see Figure 8).

One source, $\mathrm{x} 286$, has very soft X-ray colors which place it in the locus of the soft-state black holes. The remaining sources are consistent with intermediate-accretion state $\mathrm{BH}$ binaries or NS LMXBs. We note that $\mathrm{x} 403$ has been reported as a ULX candidate before (Immler et al. 1999; Stobbart et al. 2006; Ducci et al. 2013); the current NUSTAR observed colors and intensities suggest that this source is more consistent with a soft- or intermediate-accretion state BH binary. Unfortunately, we only have reliable $N U S T A R$ fluxes for $\mathrm{x} 403$ from epoch 2 due to the stray light contamination from IC 4239A. We measure an $L_{X}$ of $2 \times 10^{39} \mathrm{erg} \mathrm{s}^{-1}$ in the $0.5-8.0 \mathrm{keV}$ band with XMM-Newton, which would qualify this source as a ULX based on the observed soft X-ray luminosity (see Appendix). However, the NUSTAR properties are not consistent with other ULXs studied with NUSTAR.

\subsection{NuSTAR Source Variability}

Accreting binary populations are highly variable and NUSTAR has detected statistically significant variations in the $\mathrm{X}$-ray binary populations in the NGC 253 monitoring campaign on timescales of several weeks to several months (Lehmer et al. 2013; Wik et al. 2014b). Here, we extend this 4-25 keV variability analysis to M83 on 4-6 month timescales.

Source x299 changed in brightness significantly over the three epochs of the NUSTAR observations $(90 \%$ error 
Table 3

M83 NuSTAR Point Sources

\begin{tabular}{|c|c|c|c|c|c|c|c|c|c|c|c|c|}
\hline \multirow{3}{*}{$\begin{array}{l}\mathrm{ID}^{\mathrm{a}} \\
\mathrm{L} 14\end{array}$} & \multicolumn{4}{|c|}{ Epoch 1} & \multicolumn{4}{|c|}{ Epoch 2} & \multicolumn{4}{|c|}{ Epoch 3} \\
\hline & \multicolumn{4}{|c|}{ Count Rates } & \multicolumn{4}{|c|}{ Count Rates } & \multicolumn{4}{|c|}{ Count Rates } \\
\hline & $(4-6 \mathrm{keV})$ & $(6-12 \mathrm{keV})$ & $(12-25 \mathrm{keV})$ & $(4-25 \mathrm{keV})$ & $(4-6 \mathrm{keV})$ & $(6-12 \mathrm{keV})$ & $(12-25 \mathrm{keV})$ & $(4-25 \mathrm{keV})$ & $(4-6 \mathrm{keV})$ & $(6-12 \mathrm{keV})$ & $(12-25 \mathrm{keV})$ & $(4-25 \mathrm{keV})$ \\
\hline $\mathrm{x} 281$ & $0.27_{-0.18}^{+0.21}$ & $0.62_{-0.23}^{+0.25}$ & $0.23_{-0.19}^{+0.21}$ & $1.40_{-0.37}^{+0.39}$ & $0.57_{-0.24}^{+0.27}$ & $0.70_{-0.28}^{+0.32}$ & $0.51_{-0.27}^{+0.31}$ & $1.39_{-0.45}^{+0.49}$ & $0.52_{-0.22}^{+0.22}$ & $0.45_{-0.21}^{+0.23}$ & $<0.15$ & $0.75_{-0.32}^{+0.34}$ \\
\hline x029 & $1.18_{-0.23}^{+0.25}$ & $1.03_{-0.25}^{+0.27}$ & $<0.16$ & $2.35_{-0.39}^{+0.42}$ & $\cdots$ & $\cdots$ & $\cdots$ & $\cdots$ & $0.95_{-0.26}^{+0.28}$ & $1.14_{-0.27}^{+0.29}$ & $<0.32$ & $2.08_{-0.41}^{+0.43}$ \\
\hline x048 & $<0.16$ & $<0.17$ & $<0.26$ & $<0.55$ & $0.29_{-0.24}^{+0.29}$ & $0.33_{-0.25}^{+0.30}$ & $<0.43$ & $0.93_{-0.46}^{+0.51}$ & $0.21_{-0.13}^{+0.19}$ & $0.36_{-0.16}^{+0.18}$ & $<0.15$ & $0.53_{-0.25}^{+0.28}$ \\
\hline $\mathrm{x} 073$ & $0.18_{-0.13}^{+0.16}$ & $0.22_{-0.16}^{+0.18}$ & $<0.16$ & $0.43_{-0.26}^{+0.29}$ & $<0.36$ & $0.48_{-0.30}^{+0.35}$ & $<0.59$ & $1.03_{-0.47}^{+0.53}$ & $0.19_{-0.15}^{+0.18}$ & $<0.32$ & $<0.20$ & $0.35_{-0.26}^{+0.28}$ \\
\hline x145 & $0.58_{-0.21}^{+0.23}$ & $0.66_{-0.24}^{+0.26}$ & $<0.35$ & $1.48_{-0.38}^{+0.40}$ & $0.49_{-0.24}^{+0.28}$ & $0.57_{-0.27}^{+0.31}$ & $0.41_{-0.25}^{+0.29}$ & $1.11_{-0.44}^{+0.48}$ & $0.45_{-0.23}^{+0.23}$ & $0.53_{-0.22}^{+0.24}$ & $<0.25$ & $0.99_{-0.34}^{+0.37}$ \\
\hline x165 & $0.22_{-0.17}^{+0.20}$ & $0.65_{-0.22}^{+0.25}$ & $<0.22$ & $1.10_{-0.35}^{+0.37}$ & $0.27_{-0.20}^{+0.24}$ & $0.44_{-0.27}^{+0.32}$ & $0.29_{-0.25}^{+0.30}$ & $0.66_{-0.39}^{+0.44}$ & $0.53_{-0.24}^{+0.22}$ & $0.48_{-0.20}^{+0.22}$ & $<0.31$ & $1.09_{-0.33}^{+0.35}$ \\
\hline $\mathrm{x} 152$ & $0.46_{-0.18}^{+0.20}$ & $0.53_{-0.20}^{+0.22}$ & $<0.15$ & $1.11_{-0.32}^{+0.34}$ & $0.43_{-0.24}^{+0.29}$ & $0.49_{-0.28}^{+0.34}$ & $0.34_{-0.27}^{+0.32}$ & $1.03_{-0.46}^{+0.51}$ & $0.68_{-0.23}^{+0.19}$ & $0.68_{-0.20}^{+0.22}$ & $<0.15$ & $1.30_{-0.30}^{+0.32}$ \\
\hline x155 & $0.62_{-0.25}^{+0.28}$ & $0.54_{-0.27}^{+0.31}$ & $0.33_{-0.26}^{+0.29}$ & $1.45_{-0.46}^{+0.49}$ & $<0.39$ & $0.60_{-0.30}^{+0.34}$ & $0.43_{-0.28}^{+0.32}$ & $0.59_{-0.43}^{+0.48}$ & $<0.18$ & $<0.36$ & $<0.24$ & $<0.39$ \\
\hline x185 & $1.46_{-0.27}^{+0.26}$ & $1.13_{-0.26}^{+0.28}$ & $<0.39$ & $2.94_{-0.43}^{+0.45}$ & $1.22_{-0.29}^{+0.33}$ & $0.95_{-0.31}^{+0.35}$ & $0.82_{-0.30}^{+0.34}$ & $2.28_{-0.49}^{+0.52}$ & $1.56_{-0.27}^{+0.32}$ & $1.41_{-0.26}^{+0.28}$ & $0.24_{-0.17}^{+0.19}$ & $3.17_{-0.41}^{+0.43}$ \\
\hline x193 & $1.23_{-0.42}^{+0.41}$ & $1.46_{-0.43}^{+0.45}$ & $<0.20$ & $2.60_{-0.64}^{+0.66}$ & $0.61_{-0.48}^{+0.52}$ & $1.02_{-0.54}^{+0.59}$ & $0.87_{-0.52}^{+0.57}$ & $1.54_{-0.79}^{+0.84}$ & $1.16_{-0.45}^{+0.52}$ & $1.01_{-0.42}^{+0.44}$ & $<0.49$ & $2.35_{-0.64}^{+0.66}$ \\
\hline $\mathrm{x} 252$ & $0.25_{-0.20}^{+0.23}$ & $0.62_{-0.28}^{+0.31}$ & $<0.47$ & $1.12_{-0.43}^{+0.47}$ & $0.39_{-0.21}^{+0.25}$ & $0.83_{-0.32}^{+0.36}$ & $0.62_{-0.30}^{+0.34}$ & $1.46_{-0.48}^{+0.52}$ & $0.48_{-0.24}^{+0.26}$ & $0.43_{-0.26}^{+0.29}$ & $<0.36$ & $1.00_{-0.40}^{+0.43}$ \\
\hline $\mathrm{x} 246$ & $1.13_{-0.49}^{+0.47}$ & $0.82_{-0.48}^{+0.51}$ & $<0.40$ & $2.24_{-0.75}^{+0.78}$ & $1.14_{-0.59}^{+0.65}$ & $<0.61$ & $<0.61$ & $1.15_{-0.87}^{+0.94}$ & $0.81_{-0.47}^{+0.36}$ & $0.38_{-0.33}^{+0.36}$ & $<0.23$ & $1.09_{-0.53}^{+0.56}$ \\
\hline $\mathrm{x} 233$ & $1.63_{-0.49}^{+0.61}$ & $2.18_{-0.56}^{+0.60}$ & $0.37_{-0.25}^{+0.28}$ & $4.24_{-0.83}^{+0.86}$ & $1.75_{-0.66}^{+0.73}$ & $2.49_{-0.70}^{+0.76}$ & $2.49_{-0.70}^{+0.75}$ & $4.56_{-1.05}^{+1.11}$ & $2.26_{-0.63}^{+0.71}$ & $2.25_{-0.56}^{+0.59}$ & $<0.59$ & $4.77_{-0.86}^{+0.89}$ \\
\hline x248 & $1.20_{-0.25}^{+0.26}$ & $0.62_{-0.22}^{+0.24}$ & $<0.32$ & $2.28_{-0.39}^{+0.41}$ & $1.10_{-0.33}^{+0.37}$ & $0.90_{-0.35}^{+0.40}$ & $0.74_{-0.33}^{+0.38}$ & $2.48_{-0.58}^{+0.63}$ & $1.43_{-0.30}^{+0.27}$ & $0.58_{-0.21}^{+0.23}$ & $0.22_{-0.16}^{+0.18}$ & $2.21_{-0.37}^{+0.39}$ \\
\hline $\mathrm{x} 258$ & $0.59_{-0.19}^{+0.20}$ & $1.03_{-0.24}^{+0.26}$ & $<0.23$ & $2.25_{-0.37}^{+0.39}$ & $0.92_{-0.45}^{+0.55}$ & $1.32_{-0.58}^{+0.69}$ & $0.98_{-0.54}^{+0.65}$ & $3.27_{-0.98}^{+1.08}$ & $0.90_{-0.24}^{+0.19}$ & $1.34_{-0.23}^{+0.25}$ & $0.24_{-0.16}^{+0.19}$ & $2.44_{-0.35}^{+0.37}$ \\
\hline x286 & $0.65_{-0.29}^{+0.32}$ & $<0.35$ & $<0.42$ & $1.03_{-0.46}^{+0.48}$ & $0.60_{-0.44}^{+0.50}$ & $0.54_{-0.39}^{+0.46}$ & $0.48_{-0.38}^{+0.45}$ & $1.13_{-0.66}^{+0.72}$ & $<0.45$ & $<0.24$ & $<0.18$ & $<0.46$ \\
\hline x299 & $6.96_{-0.49}^{+0.49}$ & $5.74_{-0.46}^{+0.48}$ & $<0.38$ & $13.39_{-0.70}^{+0.72}$ & $1.77_{-0.40}^{+0.43}$ & $0.50_{-0.32}^{+0.36}$ & $0.34_{-0.30}^{+0.35}$ & $2.38_{-0.57}^{+0.61}$ & $4.25_{-0.35}^{+0.53}$ & $2.88_{-0.34}^{+0.35}$ & $0.20_{-0.17}^{+0.19}$ & $7.35_{-0.54}^{+0.56}$ \\
\hline $\mathrm{x} 284$ & $0.22_{-0.16}^{+0.20}$ & $<0.38$ & $0.20_{-0.19}^{+0.22}$ & $0.99_{-0.34}^{+0.36}$ & $0.17_{-0.16}^{+0.21}$ & $<0.22$ & $<0.19$ & $0.61_{-0.36}^{+0.41}$ & $0.61_{-0.20}^{+0.27}$ & $0.57_{-0.21}^{+0.23}$ & $<0.20$ & $1.19_{-0.34}^{+0.36}$ \\
\hline x321 & $1.31_{-0.28}^{+0.28}$ & $1.05_{-0.28}^{+0.30}$ & $<0.35$ & $2.97_{-0.45}^{+0.48}$ & $1.51_{-0.33}^{+0.36}$ & $0.69_{-0.27}^{+0.30}$ & $0.55_{-0.26}^{+0.29}$ & $2.27_{-0.49}^{+0.53}$ & $1.44_{-0.33}^{+0.24}$ & $0.87_{-0.23}^{+0.25}$ & $<0.19$ & $2.24_{-0.37}^{+0.39}$ \\
\hline x366 & $\ldots$ & $\ldots$ & $\ldots$ & $\ldots$ & $0.77_{-0.26}^{+0.30}$ & $0.54_{-0.26}^{+0.30}$ & $0.37_{-0.24}^{+0.28}$ & $1.19_{-0.45}^{+0.49}$ & $\ldots$ & $\ldots$ & $\ldots$ & $\ldots$ \\
\hline $\mathrm{x} 403$ & $\cdots$ & $\cdots$ & $\cdots$ & $\cdots$ & $0.70_{-0.26}^{+0.31}$ & $0.37_{-0.27}^{+0.32}$ & $<0.47$ & $1.57_{-0.50}^{+0.55}$ & $\cdots$ & $\cdots$ & $\cdots$ & $\cdots$ \\
\hline
\end{tabular}

Notes. The count rates are given in units of $10^{-3} \mathrm{ct} \mathrm{s}^{-1}$.

${ }^{\text {a }}$ Source ID by L14. 


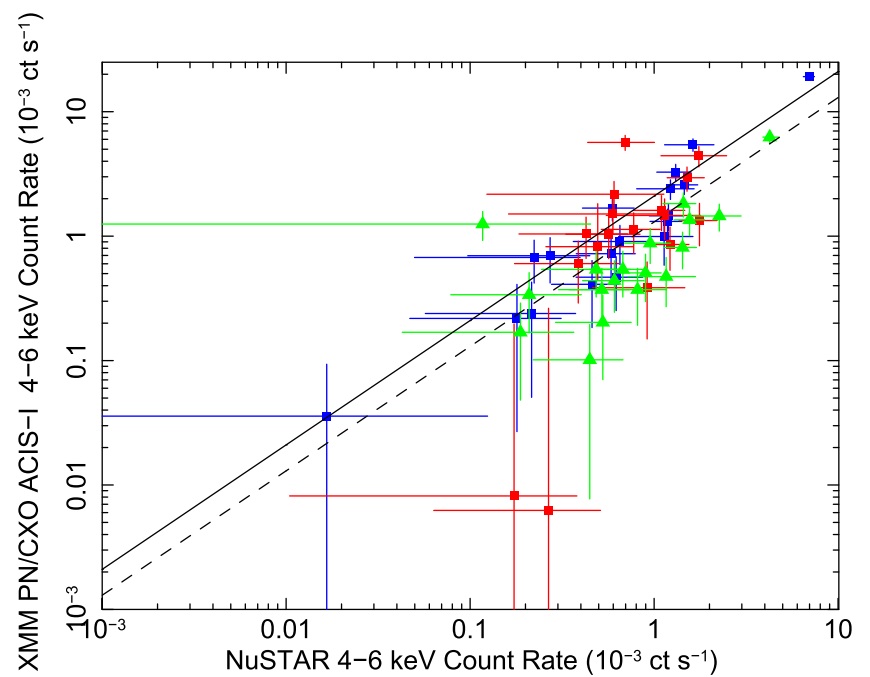

Figure 9. Count rates in the $4-6 \mathrm{keV}$ band for the same sources in the Chandra/XMM-Newton and NUSTAR observations. Red and blue data points depict NuSTARXMM-Newton (epochs 1 and 2, respectively) PN observations. Green points indicate NuSTARChandra observations. The solid line corresponds to the expected relation on count rates between XMM-Newton and $N U S T A R$ for a spectral shape of a power law with an index $\Gamma$ of 2 . The dashed line indicates the relation between Chandra and NUSTAR for the same spectral model.

confidence; see Table 3). However, the majority of sources do not show statistically significant variability. This may be due to their large statistical errors. In one of the three epochs, we have only an upper limit on the luminosity of three sources: $\mathrm{x} 155$, $\mathrm{x} 048$, and $\mathrm{x} 286$.

We also checked the variability of the NUSTAR hardness ratio (only the soft color). Figure 12 shows the variability of the five brightest sources and x155 (which shows some color variability) in the color-intensity plot. Due to the large errors in the colors of the remaining sources, they are not shown in the figure. The intensities and colors of the sources x233, x185, x321, and x258 did not change over the three epochs. x299 exhibits a possible state transition from ULX to soft/ intermediate, and back to ULX. The XMM-Newton spectral analysis (the same data sets we used and an additional observation taken in 2014 July) also suggests a similar state transition for this source (Soria et al. 2015). We present the detailed NUSTAR spectral analysis of this sources in the next section.

The source $\mathrm{x} 155$ has the same color and typical intensity as a $\mathrm{BH}-\mathrm{XRB}$ in the intermediate state in epoch 1; however, this source was observed to be harder and dimmer 5 months later in epoch 2 . Then, we only obtain an upper limit on its intensity another 5 months later in epoch 3 . The source may be changing its accretion state from intermediate, to soft, and to hard, with the soft state occurring sometime between epoch 1 and 2 . However, within the formal statistical errors, we cannot rule out that the source stays in the intermediate accretion state while changing in brightness. We note that the color and intensity in epoch 2 alone are consistent with an accreting pulsar, yet this is not likely due to the observation during epoch 1 . We also note that it is possible that the source is an NS binary.

We compare this NUSTAR intensity and color variability to the soft X-ray variabilities obtained from XMM-Newton and Chandra observations. Tables 10 and 11 list count rate, luminosity, and spectral parameters from the Chandra/XMMNewton observations for spectra with at least 200 net counts. Some sources show variability in luminosity (see Table 10). However, there are no sources showing a spectral model

Table 4

ULX (x299) 0.5-20 keV Joint Fit Results

\begin{tabular}{|c|c|c|c|c|c|c|c|}
\hline $\mathrm{Model}^{\mathrm{a}}$ & $\begin{array}{c}N_{\mathrm{H}} \\
\left(10^{21} \mathrm{~cm}^{-2}\right)\end{array}$ & $\Gamma / \Gamma_{1}^{b}$ & $\begin{array}{c}\text { Norm (PL)/ } E_{\mathrm{br}}^{\mathrm{b}} \\
\left(10^{-4}\right) /(\mathrm{keV})\end{array}$ & $\begin{array}{c}k T_{\text {in }} / \Gamma_{2}^{b} \\
(\mathrm{keV}) /-\end{array}$ & $\begin{array}{l}\text { Norm } \\
\left(10^{-4}\right)\end{array}$ & cstat/dof & $\begin{array}{c}\log L_{\mathrm{X}}{ }^{\mathrm{c}} \\
(0.5-30 \mathrm{keV}) \\
\left(\mathrm{erg} \mathrm{s}^{-1}\right)\end{array}$ \\
\hline
\end{tabular}

\begin{tabular}{|c|c|c|c|c|c|c|c|}
\hline \multicolumn{8}{|c|}{ Epoch 1} \\
\hline PL & $1.9_{-0.1}^{+0.1}$ & $1.94_{-0.04}^{+0.04}$ & $2.91_{-0.13}^{+0.14}$ & $\ldots$ & $\ldots$ & $1481.0 / 1342$ & 39.7 \\
\hline DB & $0.4_{-0.4}^{+0.0}$ & $\ldots$ & $\ldots$ & $1.52_{-0.03}^{+0.04}$ & $114.67_{-8.8}^{+9.5}$ & $1341.9 / 1342$ & 39.5 \\
\hline $\mathrm{PL}+\mathrm{DB}$ & $0.7_{-0.7}^{+0.5}$ & $2.01_{-0.26}^{+0.40}$ & $0.74_{-0.29}^{+0.40}$ & $1.63_{-0.11}^{+0.13}$ & $65.10_{-17.51}^{+18.32}$ & $1238.3 / 1340$ & 39.6 \\
\hline BPL & $0.9_{-0.2}^{+0.2}$ & $1.41_{-0.10}^{+0.10}$ & $4.1_{-0.4}^{+0.5}$ & $3.05_{-0.23}^{+0.34}$ & $2.04_{-0.14}^{+0.17}$ & $1237.8 / 1340$ & 39.6 \\
\hline
\end{tabular}

\begin{tabular}{|c|c|c|c|c|c|c|c|}
\hline \multicolumn{8}{|c|}{ Epoch 2} \\
\hline PL & $2.3_{-0.4}^{+0.5}$ & $2.50_{-0.15}^{+0.16}$ & $0.82_{-0.12}^{+0.14}$ & $\ldots$ & $\ldots$ & $525.1 / 611$ & 39.0 \\
\hline DB & $0.4_{-0.4}^{+0.2}$ & $\ldots$ & $\ldots$ & $0.92_{-0.06}^{+0.06}$ & $148.21_{-38.97}^{+69.33}$ & $524.6 / 611$ & 38.7 \\
\hline $\mathrm{PL}+\mathrm{DB}$ & $0.4_{-0.4}^{+0.2}$ & $1.85_{-1.10}^{+0.88}$ & $0.12_{-0.12}^{+0.41}$ & $0.88_{-0.11}^{+0.20}$ & $132.22_{-32.41}^{+40.29}$ & $510.3 / 609$ & 38.8 \\
\hline BPL & $2.2_{-0.7}^{+0.7}$ & $2.03_{-0.61}^{+0.27}$ & $3.5_{-1.4}^{+0.8}$ & $3.70_{-0.99}^{+1.37}$ & $0.60_{-0.13}^{+0.15}$ & $512.3 / 609$ & 38.8 \\
\hline \multicolumn{8}{|c|}{ Epoch 3} \\
\hline $\mathrm{PL}$ & $5.1_{-0.7}^{+0.7}$ & $2.41_{-0.08}^{+0.08}$ & $3.67_{-0.42}^{+0.48}$ & $\ldots$ & $\ldots$ & $788.0 / 670$ & 39.4 \\
\hline DB & $0.4_{-0.4}^{+0.3}$ & $\ldots$ & $\ldots$ & $1.53_{-0.06}^{+0.06}$ & $74.32_{-9.90}^{+12.87}$ & $662.2 / 670$ & 39.3 \\
\hline $\mathrm{PL}+\mathrm{DB}$ & $0.4_{-0.4}^{+0.4}$ & $-0.64_{-2.47}^{+2.70}$ & $0.0002_{-0.0002}^{+0.3000}$ & $1.50_{-0.07}^{+0.07}$ & $79.30_{-11.69}^{+22.30}$ & $654.4 / 668$ & 39.6 \\
\hline BPL & $2.2_{-0.7}^{+0.7}$ & $1.74_{-0.14}^{+0.13}$ & $5.0_{-0.4}^{+0.4}$ & $3.98_{-0.38}^{+0.46}$ & $1.83_{-0.26}^{+0.31}$ & $665.7 / 668$ & 39.4 \\
\hline
\end{tabular}

Notes.

${ }^{a}$ PL: power law; DB: disk blackbody; BPW: broken power law.

${ }^{\mathrm{b}}$ Broken power-law model.

${ }^{\mathrm{c}}$ Intrinsic luminosity. 


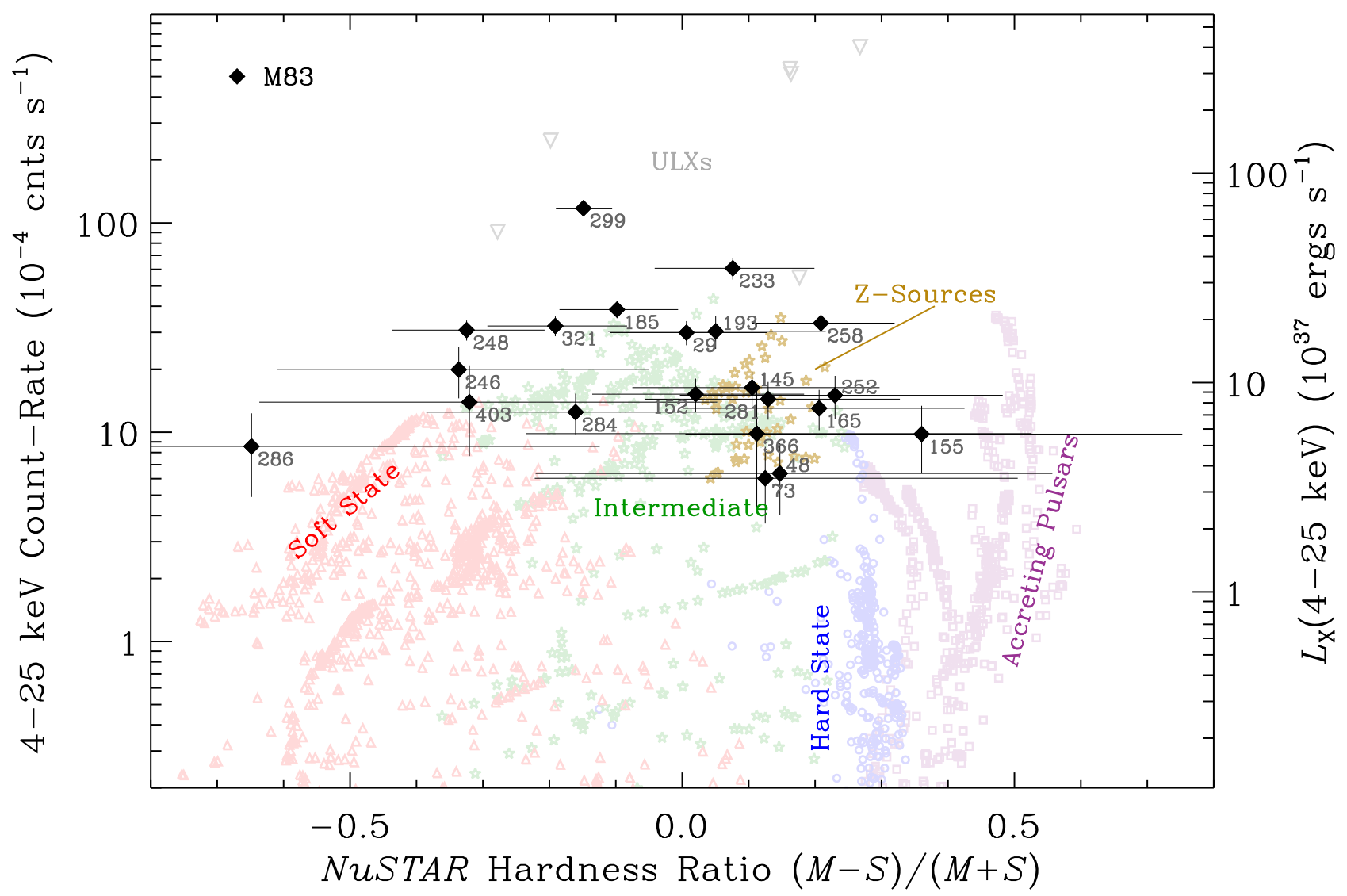

Figure 10. Hardness-intensity diagram for M83 point sources plotted with black diamonds (marked by their L14 IDs). The colors and rates are averaged over the effective exposure times. Note that the plotted count rates are scaled to the distance of $4 \mathrm{Mpc}$. The count rate of $1 \times 10^{-4} \mathrm{ct} \mathrm{s}^{-1}$ corresponds to $L_{\mathrm{X}} \sim 5 \times 10^{36} \mathrm{erg} \mathrm{s}^{-1}$. The NuSTAR soft hardness ratio is defined as $(\mathrm{M}-\mathrm{S}) /(\mathrm{M}+\mathrm{S})$, where the medium band, $\mathrm{M}$, is $6-12 \mathrm{keV}$, and the soft band, $\mathrm{S}$, is $4-6 \mathrm{keV}$. Red, green, blue, and magenta symbols depict simulated NuSTAR colors and count rates of seven Galactic LMXBs in the soft-, intermediate-, and hard-accretion state and 8 accreting Be pulsar binaries, respectively, based on RXTE spectral fits. Gray triangles and orange stars indicate the NuSTAR results for ULX sources and Galactic NS LMXBs, respectively (see text for details).

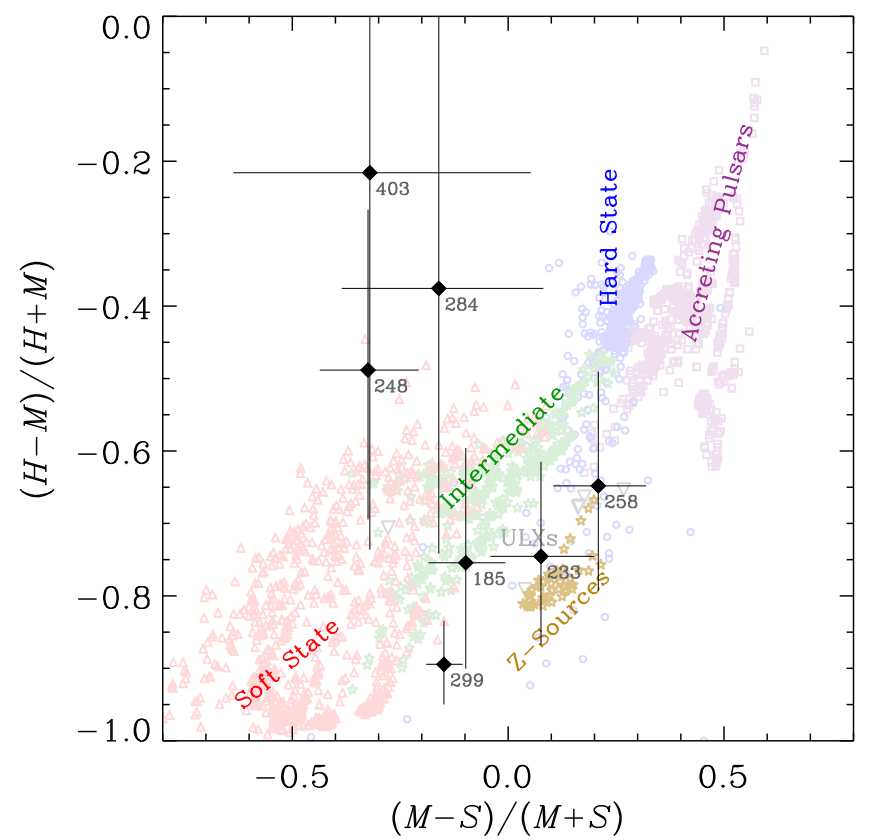

Figure 11. NUSTAR color-color diagram for the M83 point sources (black diamonds marked by their L14 IDs). The NuSTAR soft color is the same as Figure 10. The NuSTAR hard color is defined as $(\mathrm{H}-\mathrm{M}) /(\mathrm{H}+\mathrm{M})$, where the hard band, $\mathrm{H}$, is $12-25 \mathrm{keV}$. The sources with an upper limit only in the hard band are omitted. The symbols are the same as in Figure 10. change from disk blackbody to power law or vice versa, besides $\mathrm{x} 185$. For $\mathrm{x} 185$, the preferred spectral model changed from a power law to disk-blackbody model statistically, but the overall spectral shape stayed similar having either a power law of $\Gamma \sim 1.9$ or a disk blackbody with $k T \sim 1.4 \mathrm{keV}$, as well as no significant intensity change. This is consistent with the observed lack of variability for x185 in the NuSTAR hardnessintensity plot.

\section{SPECTRAL ANALYSIS}

One of the NuSTAR starburst galaxies survey goals is to characterize the galaxy-wide $0.5-30 \mathrm{keV}$ spectral properties of nearby star-forming galaxies, which ultimately are used to calibrate the k-corrections for high- $z$ galaxies (e.g., Lehmer et al. 2016). In this section, we perform joint spectral analysis using NUSTAR and XMM-Newton/Chandra to investigate the spectral properties of the brightest point sources along with the integrated galaxy spectra.

For the NuSTAR data, the source spectra and spectral response files (i.e., ARFs and RMFs) were created by the script nuproducts. We used the $C I A O$ specextract script to create the corresponding files for the Chandra data. We utilized SAS arfgen and rmfgen to create the XMMNewton response files.

We note that the background spectra for the Chandra data were created using a local background extracted from the 


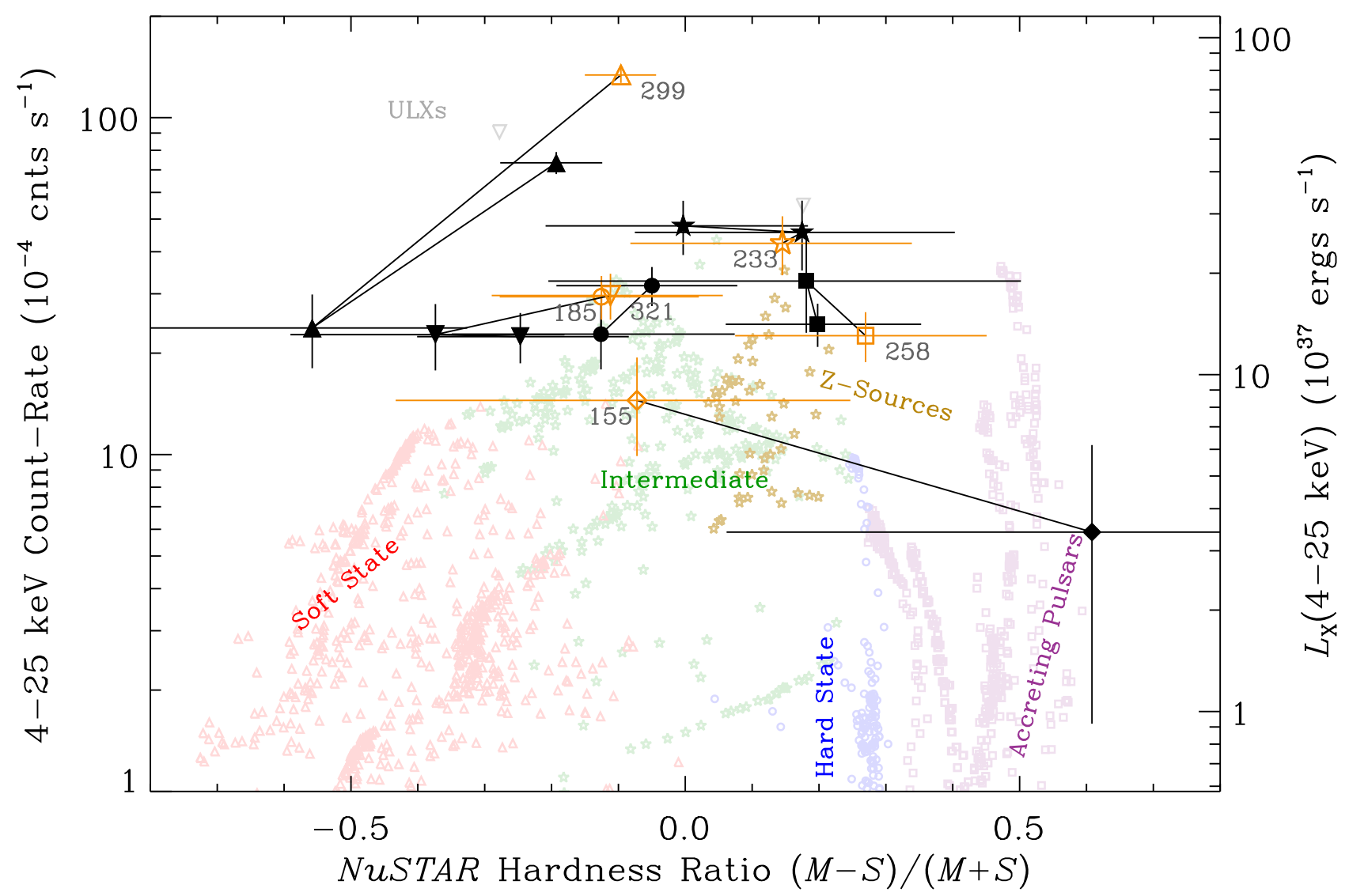

Figure 12. Hardness-intensity diagram for the five brightest sources and $x 155$. Colors and symbols for the Galactic sources and ULXs are the same as in Figure 10 . M83 sources are indicated in orange or black (epoch 1 or epochs 2 and 3, respectively) with the L14 source ID.

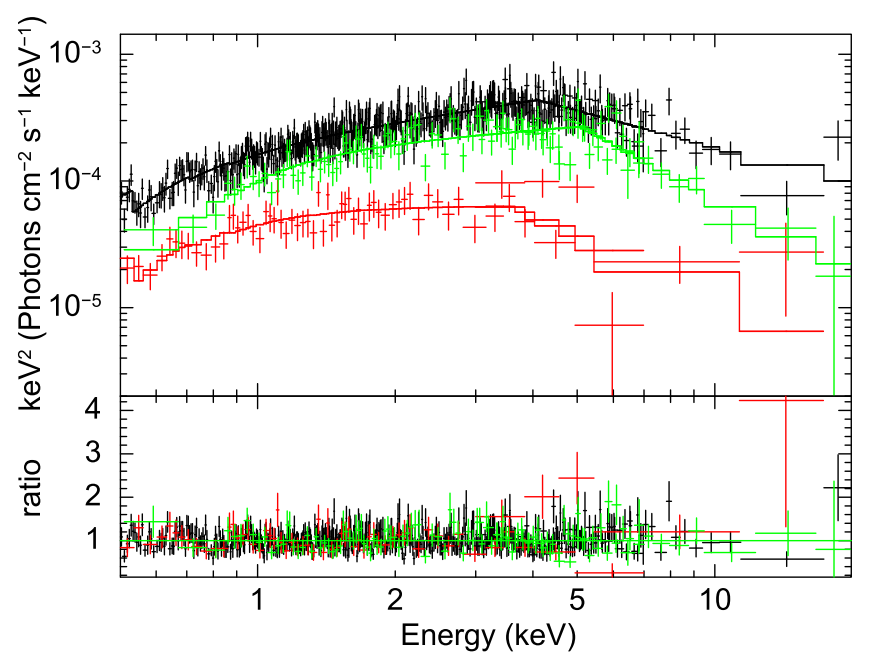

Figure 13. Unfolded $0.5-20 \mathrm{keV}$ joint Chandra/XMM-Newton and NuSTAR spectra and fitted broken power-law model in $E^{2} f(E)$ and data to model ratio of x299 (ULX) for epochs 1 (black), 2 (red), and 3 (green). All spectra show a break at $3-5 \mathrm{keV}$. This is consistent with other ULXs studied with NuSTAR.

vicinity of the source regions in the observation. Local backgrounds were also used for the XMM-Newton point source analysis; however, we used the ESAS analysis methods for the integrated galaxy analysis. ${ }^{20}$ Specifically, the instrument

$\overline{20}$ http://heasarc.gsfc.nasa.gov/docs/xmm/xmmhp_xmmesas.html background was subtracted, but the soft proton, as well as foreground and cosmic background, were modeled during the fitting process. In order to characterize the soft proton, foreground, and cosmic backgrounds, we include a source-free spectrum using a $7^{\prime}-10^{\prime}$ annulus around the galaxy center during the fitting procedure. As discussed in Wik et al. (2014a), the NUSTAR background strongly depends on detector position. Therefore, a local background is often not adequate for NuSTAR spectral analysis. We therefore obtained NuSTAR background spectra using nuskybgd.

The NUSTAR and XMM-Newton source spectra were grouped to have at least one count per spectral bin (see Wik et al. 2014a), whereas the Chandra spectra were not grouped. The joint fitting was performed in XSPEC using the C-statistic. The fitted energy is $0.5-7.0 \mathrm{keV}$ for Chandra and $X M M$ Newton (although the $0.5-12.0 \mathrm{keV}$ bandpass was used for the XMM-Newton ESAS analysis), and 3.0-20.0 keV for NuSTAR. The galaxy is not a strong hard X-ray emitter, and NuSTAR has strong instrumental line emission in the range $20-30 \mathrm{keV}$; therefore, we exclude data above $20 \mathrm{keV}$ instead of attempting to model the complicated line emission. Except for the integrated galaxy spectra for epoch 1 (see next section for detail), we co-added spectra of obsids 50002043002 and 50002043004 for epoch 1 and obsids 50002043010 and 50002043012 for epoch 3 using the FTOOL addascaspec in order to increase the $\mathrm{S} / \mathrm{Ns}$.

\subsection{ULX: $x 299$}

This source has the highest average luminosity over three epochs in the NuSTAR band (see Table 2). The source was 


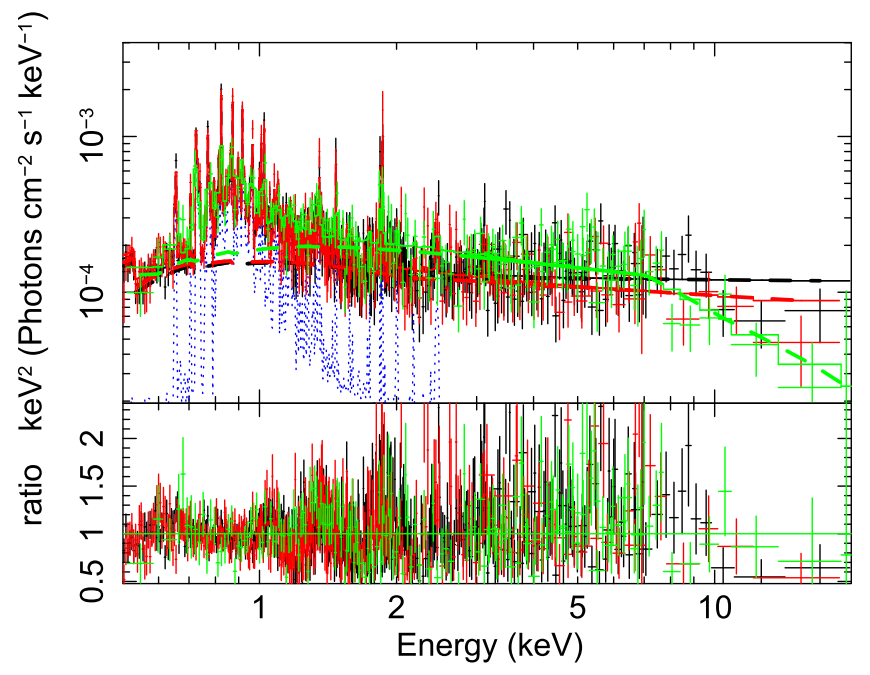

Figure 14. Unfolded data and fitted absorbed broken power-law plus thermal gas model in $E^{2} f(E)$ and ratio for the nuclear 20" radius region of M83 for epochs 1 (black), 2 (red), and 3 (green). The thick dashed lines indicate the broken power-law component and the blue dotted line represents the thermal gas emission. The spectra of the point sources in the nuclear region became steeper at the hard energies, especially in epoch 3. However, the statistical uncertainties are large above $10 \mathrm{keV}$.

discovered in 2010 with Chandra (Soria et al. 2012) as a ULX reaching $L_{\mathrm{X}} \sim 5 \times 10^{39} \mathrm{erg} \mathrm{s}^{-1}$ in 2011 in the $0.3-10 \mathrm{keV}$ band. It has been bright for close to four years, showing some color and intensity variability (Soria et al. 2015). The detailed $X M M-N e w t o n$ spectral analysis applying various models is published in Soria et al. (2015). We note that the third observation in Soria et al. (2015) was done with XMM-Newton in 2014 July, which is about a month after our epoch 3 observation (with Chandra), although the first two XMMNewton observations in their paper are also used in this work. Within a month, the source showed color variability, becoming harder in the Swift color band as shown in the Figure 2 of Soria et al. (2015). In summary, the high $\mathrm{S} / \mathrm{N} X M M-N e w t o n$ spectra taken in 2013 and 2014 show a curvature, although the Chandra spectra in 2010-2011 were dominated by a powerlaw component. Soria et al. (2015) concluded that the XMMNewton spectra were consistent with a slim disk model and that the source is varying between the ultraluminous and soft/high states.

In this paper, we jointly fit NUSTAR and Chandra/XMMNewton spectra over the $0.5-20 \mathrm{keV}$ band, applying four models to this source: an absorbed power law (PL), absorbed disk blackbody (DB), absorbed power law plus disk blackbody $(\mathrm{PL}+\mathrm{DB})$, or an absorbed broken power law (BPL). The resulting fit parameters are tabulated in Table 4. Overall, an absorbed power-law model is not preferred for epochs 1 and 3 . However, an absorbed power-law model and an absorbed diskblackbody model give comparable results (C-stat/degrees of freedom (dof) of 525.1/611 and 524.6/611, respectively) during epoch 2 when the source is at its faintest level $\left(L_{X} \sim\right.$ $\left.(5-10) \times 10^{38} \mathrm{erg} \mathrm{s}^{-1}\right)$. The power-law model was not preferred during epoch 2 when fitting only XMM-Newton data as shown in Table 11. Soria et al. (2015) also reported that a power-law model was rejected during the 2013-2014 XMMNewton observations. NUSTAR could be detecting a power-lawlike spectrum at $10-20 \mathrm{keV}$ where XMM-Newton is not sensitive.

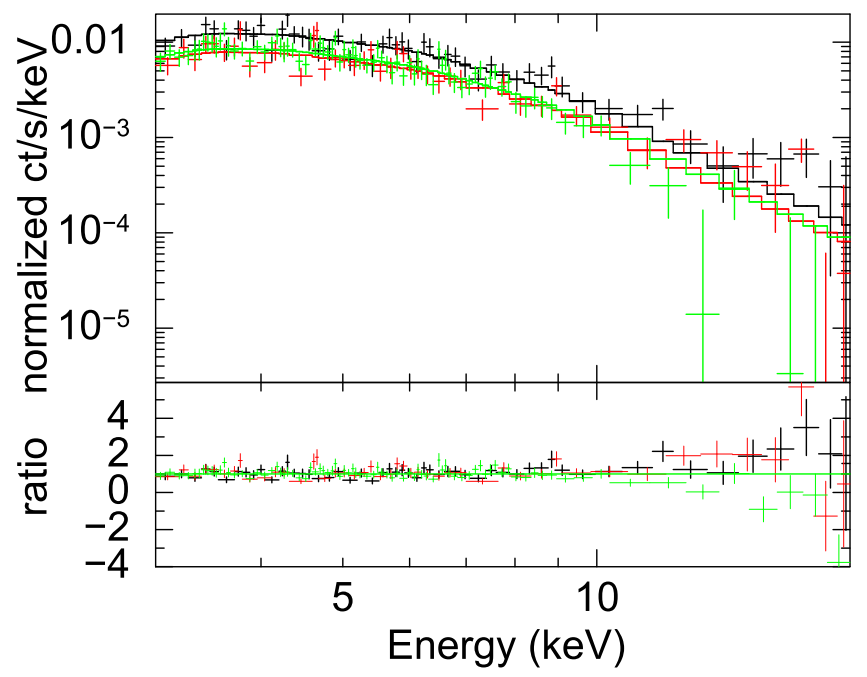

Figure 15. NUSTAR spectra of the integrated M83 galaxy. Black, red, and green indicate the spectrum for epochs 1,2, and 3, respectively. A broken power-law model was fitted simultaneously tying the power-law indices. The obtained parameters are $\Gamma_{1} \sim 2.3$ (for the lower energy) and $\Gamma_{2} \sim 3.0$ (for the higher energy) with the break at $\sim 6 \mathrm{keV}$. These values are consistent with the starburst galaxy NGC 253. The spectra have been rebinned to achieve at least $4 \sigma$ for display purposes.

We also tried an absorbed disk-blackbody plus power-law model. During epoch 1, the second component (power law) provides statistically significant improvement to the fit ( $\Delta C>100$ for 2 additional dof). However, during epochs 2 and 3 , the additional component only improves $\Delta C \sim 15$. The obtained fitted parameter values for epochs 1 and 2 are consistent with Soria et al. (2015). Although the power-law component for epoch 3 is not well constrained by our Chandra spectrum, our result for epoch 3 agrees with Soria et al. (2015) within the errors.

We performed a statistical test using XSPEC simftest with 1000 trials to see whether or not a power-law component is required during epochs 1 and 2. The Chandra spectrum does not have sufficient $\mathrm{S} / \mathrm{N}$ to confirm this, and so we do not perform the test for the lower $\mathrm{S} / \mathrm{N}$ epoch 3. During epoch 1 , both XMM-Newton only and joint NUSTAR analyses require a power-law component in addition to a disk-blackbody component. The power-law component was required at the $1 \sigma$ level during epoch 2 with the XMM-Newton only analysis. However, this power-law component was required at more than $3 \sigma$ when including NuSTAR data. We note that below $5 \mathrm{keV}$, the disk-blackbody component dominates. Overall, the ULX shows spectral properties which are very similar to $\mathrm{BH}$ binaries in the intermediate accretion state.

In order to compare the $\mathrm{x} 299$ spectral features to the integrated galaxy in Section 4.3, we also fitted with an absorbed broken power-law model, which also fits the data well. Figure 13 shows the unfolded broken power-law joint NuSTAR and Chandra/XMM-Newton spectra of x299 for each epoch, exhibiting how spectral shapes are changing over three epochs.

\subsection{Nucleus}

The nuclear region of M83 also appears as a bright $N u S T A R$ source. The high spatial resolution Chandra images already reveal that there are several bright X-ray point sources near the center of M83 (see Figure 8). However, these sources are not 
Table 5

Nuclear Region 0.5-20 keV Joint Fit Results

\begin{tabular}{lcccccccc}
\hline \hline $\begin{array}{l}\text { Model } \\
+2 \text { apec }\end{array}$ & $\begin{array}{c}N_{\mathrm{H}} \\
\left(10^{21} \mathrm{~cm}^{-2}\right)\end{array}$ & $\Gamma / \Gamma_{1}^{b}$ & $\begin{array}{c}E_{\mathrm{br}}^{\mathrm{b}} \\
(\mathrm{keV})\end{array}$ & $\begin{array}{c}k T_{\mathrm{in}} / \Gamma_{2}{ }^{\mathrm{b}} \\
(\mathrm{keV}) /-\end{array}$ & $\begin{array}{c}\text { Norm } \\
\left(10^{-4}\right)\end{array}$ & $\begin{array}{c}k T_{e 1} \\
(\mathrm{keV})\end{array}$ & $\begin{array}{l}\text { Norm } \\
\left(\mathrm{gas}_{1}\right) \\
\left(10^{-4}\right)\end{array}$ & $\begin{array}{c}k T_{e 2} \\
\left(\mathrm{keV}^{2}\right)\end{array}$ \\
\hline
\end{tabular}

\begin{tabular}{lccccccccc}
\hline \multicolumn{10}{c}{ Epoch 1} \\
\hline PL & $0.5_{-0.2}^{+0.2}$ & $2.11_{-0.07}^{+0.07}$ & $\ldots$ & $\ldots$ & $1.44_{-0.12}^{+0.13}$ & $0.59_{-0.14}^{+0.09}$ & $0.69_{-0.20}^{+0.31}$ & $0.97_{-0.07}^{+0.16}$ & $0.84_{-0.32}^{+0.21}$ \\
DB & 0.4 & $\ldots$ & $\ldots$ & $1.58_{-0.10}^{+0.11}$ & $37.8_{-8.7}^{+11.2}$ & $0.32_{-0.01}^{+0.01}$ & $1.13_{-0.08}^{+0.08}$ & $0.96_{-0.02}^{+0.02}$ & $1.14_{-0.07}^{+0.07}$ \\
BPL & 0.4 & $2.10_{-0.06}^{+0.05}$ & 9.05 & $2.11_{-0.76}^{+0.94}$ & $1.43_{-0.08}^{+0.08}$ & $0.57_{-0.10}^{+0.09}$ & $0.07_{-0.17}^{+0.33}$ & $0.96_{-0.06}^{+0.14}$ & $0.09_{-0.33}^{+0.16}$
\end{tabular}

Epoch 2

\begin{tabular}{lccccccccc}
\hline PL & 0.4 & $2.21_{-0.06}^{+0.06}$ & $\ldots$ & $\ldots$ & $1.53_{-0.11}^{+0.11}$ & $0.62_{-0.14}^{+0.09}$ & $0.79_{-0.33}^{+0.28}$ & $0.98_{-0.10}^{+0.18}$ & $0.73_{-0.36}^{+0.30}$ \\
DB & 0.4 & $\ldots$ & $\ldots$ & $1.44_{-0.12}^{+0.13}$ & $51.6_{-15.1}^{+20.8}$ & $0.31_{-0.02}^{+0.02}$ & $1.20_{-0.11}^{+0.11}$ & $0.94_{-0.03}^{+0.03}$ & $1.41_{-0.10}^{+0.10}$ \\
BPL & 0.4 & $2.20_{-0.07}^{+0.07}$ & 10.3 & $3.84_{-1.70}^{+2.76}$ & $1.51_{-0.12}^{+1.13}$ & $0.61_{-0.14}^{+0.09}$ & $0.76_{-0.30}^{+0.31}$ & $0.96_{-0.09}^{+0.18}$ & $0.96_{-0.36}^{+0.28}$ \\
\hline \multicolumn{7}{c}{ Epoch 3} \\
\hline PL & 0.4 & $2.37_{-0.08}^{+0.08}$ & $\ldots$ & $\ldots$ & $2.37_{-0.26}^{+0.28}$ & $0.54_{-0.17}^{+0.48}$ & $0.37_{-0.33}^{+0.54}$ & $0.96_{-0.64}^{+0.38}$ & $0.77_{-0.34}^{+0.21}$ \\
DB & 0.4 & $\ldots$ & $\ldots$ & $1.91_{-0.19}^{+0.23}$ & $16.0_{-6.8}^{+1.5}$ & $0.62_{-0.06}^{+0.15}$ & $1.30_{-0.14}^{+0.15}$ & $1.57_{-0.32}^{+0.25}$ & $2.14_{-0.49}^{+0.27}$ \\
BPL & 0.4 & $2.21_{-0.14}^{+0.10}$ & $6.91_{-1.96}^{+0.91}$ & $3.64_{-0.80}^{+0.92}$ & $2.06_{-0.30}^{+0.29}$ & $0.54_{-0.19}^{+0.25}$ & $0.53_{-0.32}^{+0.35}$ & $1.00_{-0.12}^{+0.17}$ & $0.83_{-0.61}^{+0.24}$ \\
\hline
\end{tabular}

Notes.

a PL: power law; DB: disk blackbody; BPL: broken power law; a solar abundance (Anders \& Grevesse 1989) is assumed for the apec component.

${ }^{\mathrm{b}}$ Broken power-law model.

Table 6

Nuclear Region Fit Statistics and 0.5-30 keV Luminosities

\begin{tabular}{|c|c|c|c|c|}
\hline $\begin{array}{l}\text { Model }^{\mathrm{a}} \\
+2 \text { apec }\end{array}$ & C-stat/dof & $\begin{array}{l}\log L_{X}^{\text {obs }} \\
\left(\mathrm{erg} \mathrm{s}^{-1}\right)\end{array}$ & $\begin{array}{l}\log L_{X}^{\text {int }} \\
\left(\operatorname{erg~s}^{-1}\right)\end{array}$ & $\begin{array}{c}\log L_{\mathrm{X} \text { gas }}^{\text {int }} \\
\left(\mathrm{erg} \mathrm{s}^{-1}\right)\end{array}$ \\
\hline \multicolumn{5}{|c|}{ Epoch 1} \\
\hline PL & $1017.6 / 1112$ & 39.5 & 39.5 & 39.0 \\
\hline DB & $1266.9 / 1113$ & 39.4 & 39.5 & 39.2 \\
\hline BPL & $1014.8 / 1112$ & 39.4 & 39.5 & 39.0 \\
\hline \multicolumn{5}{|c|}{ Epoch 2} \\
\hline PL & $793.4 / 905$ & 39.4 & 39.5 & 39.0 \\
\hline DB & $921.6 / 905$ & 39.4 & 39.4 & 39.2 \\
\hline BPL & $790.9 / 904$ & 39.4 & 39.5 & 39.0 \\
\hline \multicolumn{5}{|c|}{ Epoch 3} \\
\hline PL & $721.6 / 674$ & 39.5 & 39.5 & 38.9 \\
\hline DB & $775.1 / 674$ & 39.4 & 39.5 & 39.2 \\
\hline BPL & $703.5 / 672$ & 39.5 & 39.5 & 39.0 \\
\hline
\end{tabular}

Note.

${ }^{a}$ PL: power law; DB: disk blackbody; BPL: broken power law.

resolved by the NUSTAR PSF. At most, we were able to identify via PSF fitting two NuSTAR (x233 and x193) sources, separated by 15 arcsec. Yet, these two sources cannot be separated when extracting spectra. Therefore, we utilize a $20^{\prime \prime}$ radius aperture (shown in Figure 8) to capture both sources with as much flux as possible for spectral analysis while avoiding contamination from $\mathrm{x} 299$. In order to be consistent with the NUSTAR source spectra, we extract the XMM-Newton and Chandra spectra using the same $20^{\prime \prime}$ radius aperture as the NUSTAR aperture. Aperture corrections were applied to the NuSTAR and XMM-Newton data, since the sizes of the PSFs for these telescopes are larger than $20^{\prime \prime}$. For the Chandra data, we used area-weighted ARFs because Chandra's PSF size is much smaller than $20^{\prime \prime}$.

We applied three (PL, DB, BPL) models for the nuclear region but added a thermal gas component using a two temperature model (XSPEC apec + apec) to account for the strong, diffuse soft X-ray emission seen in the Chandra data. The fitting results and luminosities for the nuclear region are tabulated in Tables 5 and 6.

For the two temperature model, the fitting suffered from a degeneracy between the column density and thermal gas temperatures, resulting in a high $N_{\mathrm{H}}$ and a lower temperature. Therefore, we fixed the column density $N_{\mathrm{H}}$ to the Galactic value $\left(4 \times 10^{20} \mathrm{~cm}^{-2}\right.$; Dickey \& Lockman 1990). The obtained temperatures are $0.3-0.6 \mathrm{keV}$ and $0.9-1.5 \mathrm{keV}$ (see Table 5). The lower temperature is consistent with what Soria \& $\mathrm{Wu}$ (2002) found $(k T=0.6 \mathrm{keV})$ in the nuclear $16^{\prime \prime}$ starburst region using Chandra. We note that the second temperature is less constrained for the Chandra spectrum due to the poor $\mathrm{S} / \mathrm{N}$ compared to the XMM-Newton data. The higher temperature $(\sim 1 \mathrm{keV})$ is higher than those typically measured in the galaxywide spectra of nearby star-forming galaxies with Chandra (Mineo et al. 2012b). However, we limit our study to the nuclear region in M83 where intense star formation is ongoing. It is possible that we detect a very hot gas component that is directly associated with supernova ejecta (Strickland \& Heckman 2007) as seen, for example, in the central region of M82.

Contrary to $\mathrm{x} 299$, a power-law model with a steeper slope of $\Gamma \sim 2.0-2.5$ is preferred to a disk-blackbody model. This is similar to the results obtained for the resolved nuclear sources x233 and x193 in Table B using Chandra and XMM-Newton alone. The luminosity in the $0.5-30 \mathrm{keV}$ band does not change over the three epochs.

An absorbed broken power-law also fits well, but it does not significantly improve the fit statistics. For epochs 1 and 2, residuals above $10 \mathrm{keV}$ from a single power law indicate a break. When an absorbed broken power law is applied, the 
Table 7

Integrated Galaxy 0.5-20 keV Joint Fit Results

\begin{tabular}{lcccccccc}
\hline \hline $\begin{array}{l}\text { Model } \\
+2 \text { apec }\end{array}$ & $\begin{array}{c}N_{\mathrm{H}} \\
\left(10^{21} \mathrm{~cm}^{-2}\right)\end{array}$ & $\Gamma / \Gamma_{1}^{\mathrm{b}}$ & $E_{\mathrm{br}}{ }^{\mathrm{b}}$ & $\Gamma_{2}^{\mathrm{b}}$ & $\begin{array}{c}\text { Norm } \\
\left(10^{-4}\right)\end{array}$ & $\begin{array}{c}k T_{e 1} \\
(\mathrm{keV})\end{array}$ & $\begin{array}{c}\text { Norm } \\
\left(\mathrm{gas}_{1}\right) \\
\left(10^{-4}\right)\end{array}$ & $\begin{array}{c}k T_{e 2} \\
\left(\mathrm{keV}^{2}\right)\end{array}$ \\
\end{tabular}

\begin{tabular}{|c|c|c|c|c|c|c|c|c|c|}
\hline \multicolumn{10}{|c|}{ Epoch 1} \\
\hline PL & $0.5_{-0.1}^{+0.2}$ & $1.93_{-0.03}^{+0.03}$ & $\ldots$ & ... & $9.46_{-0.46}^{+0.43}$ & $0.74_{-0.02}^{+0.02}$ & $3.72_{-0.17}^{+0.44}$ & $0.20_{-0.01}^{+0.01}$ & $4.62_{-1.40}^{+2.07}$ \\
\hline BPL & 0.4 & $1.76_{-0.08}^{+0.04}$ & $3.74_{-0.51}^{+0.42}$ & $2.27_{-0.12}^{+0.07}$ & $9.22_{-0.41}^{+0.34}$ & $0.75_{-0.01}^{+0.01}$ & $3.79_{-0.40}^{+0.19}$ & $0.20_{-0.01}^{+0.01}$ & $4.86_{-0.36}^{+0.71}$ \\
\hline \multicolumn{10}{|c|}{ Epoch 2} \\
\hline PL & $1.1_{-0.4}^{+0.3}$ & $2.23_{-0.05}^{+0.04}$ & $\ldots$ & $\ldots$ & $10.12_{-0.63}^{+0.51}$ & $0.72_{-0.02}^{+0.02}$ & $4.53_{-0.75}^{+0.66}$ & $0.19_{-0.01}^{+0.01}$ & $10.3_{-4.5}^{+4.6}$ \\
\hline BPL & $0.4_{-0.0}^{+0.3}$ & $1.85_{-0.11}^{+0.11}$ & $2.58_{-0.25}^{+0.44}$ & $2.33_{-0.08}^{+0.12}$ & $8.12_{-0.46}^{+0.53}$ & $0.75_{-0.02}^{+0.02}$ & $3.75_{-0.19}^{+0.53}$ & $0.20_{-0.01}^{+0.01}$ & $4.98_{-0.41}^{+2.41}$ \\
\hline \multicolumn{10}{|c|}{ Epoch 3} \\
\hline PL & 0.4 & $2.20_{-0.05}^{+0.05}$ & 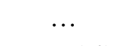 & $\ldots$ & $10.63_{-0.50}^{+0.25}$ & $0.68_{-0.07}^{+0.08}$ & $1.19_{-1.24}^{+1.58}$ & $0.27_{-0.10}^{+0.08}$ & $4.98_{-0.41}^{+2.41}$ \\
\hline BPL & 0.4 & $1.88_{-0.07}^{+0.07}$ & $5.27_{-0.49}^{+0.62}$ & $3.42_{-0.28}^{+0.40}$ & $8.44_{-0.57}^{+0.59}$ & $0.71_{-0.06}^{+0.08}$ & $3.16_{-0.99}^{+0.75}$ & $0.23_{-0.09}^{+0.10}$ & $3.26_{-1.65}^{+4.10}$ \\
\hline
\end{tabular}

Notes.

a PL: power law; BPL: broken power law; a solar abundance (Anders \& Grevesse 1989) is assumed for the apec component.

${ }^{\mathrm{b}}$ Broken power-law model.

Table 8

Integrated Galaxy Fit Statistics and 0.5-30 keV Luminosities

\begin{tabular}{|c|c|c|c|c|}
\hline $\begin{array}{l}\text { Model }^{\text {a }} \\
+2 \text { apec }\end{array}$ & C-stat/dof & $\begin{array}{l}\log L_{\mathrm{X}}^{\mathrm{obs}} \\
\left(\operatorname{erg} \mathrm{s}^{-1}\right)\end{array}$ & $\begin{array}{l}\log L_{X}^{\text {int }} \\
\left(\operatorname{erg~s}^{-1}\right)\end{array}$ & $\begin{array}{c}\log L_{\mathrm{Xgas}}^{\text {int }} \\
\left(\mathrm{erg} \mathrm{s}^{-1}\right)\end{array}$ \\
\hline \multicolumn{5}{|c|}{ Epoch 1} \\
\hline PL & $4391.3 / 4987$ & 40.3 & 40.3 & 39.6 \\
\hline BPL & $4343.5 / 4985$ & 40.3 & 40.3 & 39.7 \\
\hline \multicolumn{5}{|c|}{ Epoch 2} \\
\hline PL & $3677.0 / 4970$ & 40.2 & 40.3 & 39.8 \\
\hline BPL & $3631.3 / 4937$ & 40.2 & 40.2 & 39.6 \\
\hline \multicolumn{5}{|c|}{ Epoch 3} \\
\hline PL & $1023.2 / 862$ & 40.2 & 40.2 & 39.4 \\
\hline BPL & $862.5 / 860$ & 40.1 & 40.2 & 39.6 \\
\hline
\end{tabular}

Note.

a PL: power law; BPL: broken power law.

break energy is not constrained, and even after fixing it to the best-fit values $(9-10 \mathrm{keV})$ the parameters of the two photon indices are almost identical, indicating that the break is not supported by the data. However, for epoch 3, we find strong support for a broken power-law model. The measured break energy is $E_{\mathrm{br}}=6.91_{-1.96}^{+0.91} \mathrm{keV}$ with a steep power-law (SPL) slope at harder energies (Table 5). These spectral characteristics are similar to what we expect for ULXs, intermediate-accretion state $\mathrm{BH}$ binaries, and NS binaries (Z-sources; Church et al. 2012; Bachetti et al. 2013; Lehmer et al. 2013; Walton et al. 2013, 2014).

Since the nuclear region spectra are a collection of multiple sources, we do not interpret the fitted parameter values physically. Instead, we are curious how the spectral shape of the nuclear region varies over three epochs. The unfolded Chandra/XMM-Newton and NUSTAR spectra of the nuclear region in $E^{2} f(E)$ for each epoch are shown in Figure 14. The nuclear spectra are more or less consistent over the three epochs. The spectrum for epoch 3 becomes steeper at harder energies; however, the statistical uncertainties are very large above $10 \mathrm{keV}$ for all three epochs.

One of NuSTAR's strengths is to constrain the presence of an obscured AGN that may not be visible in the soft X-ray bandpass with Chandra and XMM-Newton. To test this, we also applied the MyTorus model (Murphy \& Yaqoob 2009) to the nuclear region spectra. MyTorus is a model that selfconsistently computes X-ray reprocessing for a power-law component in a toroidal geometry. For the fitting, the spectral model included a power-law component absorbed by a line-ofsight component (MyTorusZ) along with reflection components (MyTorusL and MyTorusS) which would likely be due to reflection from the opposite side of the toroidal obscuration as well as scattering from gas clouds in the vicinity of the nucleus. We assumed that some scattered emission (i.e., from highly ionized, optically thin gas near the AGN) is likely present and modeled this as being the same power law as used with MyTorus but with the normalization fixed at $30 \%$ of the main power law (i.e., $30 \%$ of the AGN flux is scattered around the torus). Finally, we included components for thermal (XSPEC apec) and a second absorbed power law to account for the X-ray binaries. All of the components included optically thin "global" absorption (i.e., toward the M83 system and within the extranuclear region).

To obtain a better constraint, we jointly fit three epoch spectra. We fixed global $N_{\mathrm{H}}$ to $5 \times 10^{20} \mathrm{~cm}^{-2}$ as we used for the previous fits. The fitted thermal component temperatures were $\sim 0.62 \mathrm{keV}$ and $\sim 1.0 \mathrm{keV}$ with $L_{\mathrm{X}} \sim 1.0 \times 10^{39} \mathrm{erg} \mathrm{s}^{-1}$. The best-fit AGN power-law normalization was 0 , showing that no AGN component is required by the data. To establish the upper-limit on any intrinsic AGN luminosity, we fixed the AGN power-law slope to 1.8 and set the MyTorus $N_{\mathrm{H}}$ values at 1,2 , and $4 \times 10^{24} \mathrm{~cm}^{-2}$. We then increased the AGN powerlaw normalization until there was a change in C-statistic of 4.6. We then computed the intrinsic AGN luminosity by setting $N_{\mathrm{H}}$ to 0 , which then gave $90 \% L_{10-30 \mathrm{keV}}$ upper limits of $7.8 \times 10^{37}, 8.3 \times 10^{37}$, and $1.1 \times 10^{38} \mathrm{erg} \mathrm{s}^{-1}$ for the three $N_{\mathrm{H}}$ values from the spectral fits. The slope and 
Table 9

Observed Broken Power-law X-Ray Luminosities

\begin{tabular}{|c|c|c|c|c|c|c|}
\hline \multirow{2}{*}{ Epoch } & \multicolumn{2}{|c|}{ Galaxy } & \multicolumn{2}{|c|}{ Nucleus } & \multicolumn{2}{|c|}{ x299 } \\
\hline & $\begin{array}{c}0.5-30 \mathrm{keV} \\
\left(10^{39} \mathrm{erg} \mathrm{s}^{-1}\right)\end{array}$ & $\begin{array}{c}10-30 \mathrm{keV}^{-39} \\
\left(10^{39} \mathrm{erg} \mathrm{s}^{-1}\right)\end{array}$ & $\begin{array}{c}0.5-30 \mathrm{keV} \\
\left(10^{39} \mathrm{erg} \mathrm{s}^{-1}\right)\end{array}$ & $\begin{array}{c}10-30 \mathrm{keV}^{-1} \\
\left(10^{39} \mathrm{erg} \mathrm{s}^{-1}\right)\end{array}$ & $\begin{array}{c}0.5-30 \mathrm{keV} \\
\left(10^{39} \mathrm{erg} \mathrm{s}^{-1}\right)\end{array}$ & $\begin{array}{c}10-30 \mathrm{keV} \\
\left(10^{39} \mathrm{erg} \mathrm{s}^{-1}\right)\end{array}$ \\
\hline 1 & 15.2 & 3.5 & 1.7 & 0.30 & 4.0 & 0.45 \\
\hline 2 & 10.7 & 1.9 & 1.6 & 0.19 & 0.52 & 0.02 \\
\hline 3 & 10.4 & 0.93 & 2.1 & 0.15 & 2.0 & 0.12 \\
\hline
\end{tabular}

luminosities of the X-ray binary power-law component were $\Gamma \sim 2.2$ and $L_{\mathrm{X}} \sim 4.4 \times 10^{38} \mathrm{erg} \mathrm{s}^{-1}$, and $2.1 \times 10^{39} \mathrm{erg} \mathrm{s}^{-1}$ for $10-30 \mathrm{keV}$ and $0.5-30 \mathrm{keV}$, respectively.

\subsection{The 0.5-20 keV Integrated Galaxy Spectrum}

The integrated galaxy spectra were extracted using the $D_{25}$ area covered by the observations, excluding data within regions of significant stray light (see Figure 3). Due to the different roll angles between the observations, the size of the extraction area (see Figure 2) for the galaxy spectra were different among the six spectra (FPMA and FPMB for the three epochs). We note that all the NUSTAR sources are included in the NUSTAR spectra for all three epochs. In our NGC 253 work, we found that a small number of brightest sources dominate the integrated galaxy spectrum above $10 \mathrm{keV}$, which is characterized well as a broken power law showing rapid decrease at harder energies (Wik et al. 2014b). In this section, we examine which sources contribute to the total galaxy emission in the NuSTAR band and characterize the shape of the broadband $\mathrm{X}$-ray spectrum of M83.

First, we performed the NUSTAR-only spectral analysis, which we show in Figure 15. In general, all three epochs can be characterized well with a power law of $\Gamma \sim 2-3$, although the integrated M83 NUSTAR spectra hint at a rapid decrease at harder energies, meaning that a broken power law would result in a good fit but with poorly constrained parameters in epochs 2 and 3. We also jointly fit all three epoch spectra, tying the power-law indices but varying the normalization component. This would average the spectral shape but increase the $S / N$. A simple power-law model gives a photon index of $2.6 \pm 0.1$ with 1359/1268 (C-stat/dof). A broken power-law model gives a better fit with 1342/1266 (C-stat/dof), but the fit statistic is not significantly improved. The obtained fit parameters are $\Gamma_{1}=2.3 \pm 0.2$ and $\Gamma_{2}=3.0_{-0.2}^{+0.4}$ with a break at $6.1_{-0.9}^{+1.6} \mathrm{keV}$. These values are consistent with the integrated NuSTAR spectrum of NGC 253 (Wik et al. 2014b). Figure 15 shows the NUSTAR spectra for the integrated galaxy with the broken power-law model and data over model ratio.

For the joint NUSTAR and XMM-Newton/Chandra spectral analysis, we fit two models, either a power law or a broken power law, to the integrated galaxy spectra. Since the galaxy contains a significant amount of hot gaseous emission due to star-forming activity, a thermal plasma component is also necessary. The resulting fit parameters and luminosities are listed in Tables 7 and 8.

The measured diffuse gas temperatures are $0.65-0.75 \mathrm{keV}$ and $0.2-0.3 \mathrm{keV}$ for both models. The $0.7 \mathrm{keV}$ gas is consistent with the nuclear region; however, the second temperature is much lower compared to what we found in the spectral-fit result for the nuclear region $(\sim 1 \mathrm{keV})$. These two temperatures are consistent with what Mineo et al. (2012b) found in nearby star-forming galaxies. Unsurprisingly, the thermal gas component contribution is unchanged over three epochs. For all three epochs, the absorbed broken power-law model fits better than a single power-law model. The fit parameters for the broken power-law model are $\Gamma_{1}$ of $\sim 1.8$ and $\Gamma_{2}$ of $2-3.5$ with a break energy of $2-5.5 \mathrm{keV}$. We note that the power-law index for the harder energies and the break energy are somewhat difficult to be constrained due to low number counts in the NUSTAR spectra. Since the background/foreground components in the XMM-Newton spectra are not subtracted but modeled (see the ESAS threads and Section 4 above), we only show the models (with a broken power law) for the soft X-ray band but with the unfolded $N U S T A R$ data points to show the statistical uncertainties in the hard X-ray energies in Figure 16.

Figure 17 presents the model for the integrated galaxy spectrum for each epoch, along with the models of x299 (blue) and the nucleus (red, without thermal gas) to show how these components contribute to the integrated galaxy emission. In all of the cases, we show the absorbed broken power-law models. Source x299 shows about a factor of six change in its 0.5-30 keV luminosity, and this source effectively determines the shape of the integrated galaxy spectra, especially the location of the break energy. Specifically, the integrated galaxy spectra break around the energies where x 299 shows breaks for epochs 1 and 3 when the source is bright. During epoch 2, x299 became dim, and the integrated galaxy spectrum shows a break at $\sim 2.5 \mathrm{keV}$, whereas the nuclear region and x299 show breaks at higher energies.

Table 9 lists the observed X-ray luminosities for the pointsource contribution to the galaxy, nuclear region, and x299 at $0.5-30 \mathrm{keV}$ and $10-30 \mathrm{keV}$. The nuclear region and x299 contribute about $15 \%-30 \%$ of the total galaxy luminosity at $10-30 \mathrm{keV}$. The remaining flux is likely to be contributed by fainter point sources.

\section{DISCUSSION}

\subsection{Nature of the NuSTAR Point Sources in M83}

We have identified a total of 21 point sources in M83 with NUSTAR and all of these sources are listed in the Chandra point-source catalog compiled by L14. There are no bright hard X-ray sources that appear in the NuSTAR observations but do not have Chandra counterparts. One (x165) of these sources is classified as a background AGN based on its association with an optical counterpart (L14). It is expected that background AGNs would have NUSTAR colors similar to intermediate-state $\mathrm{BH}$ binaries, and indeed this is the case for $\mathrm{x} 165$. However, for $\mathrm{x} 165$, the NUSTAR source has a large positional offset (6".7) from the nearest Chandra counterpart, and may be a false match. We note that the number of expected background sources above a $4-25 \mathrm{keV}$ count rate of $\sim 8 \times 10^{-4} \mathrm{cts} \mathrm{s}^{-1}$ is $\sim 3.5$ for the entire $D_{25}$ based on the NuSTAR $\log N-\log S$ measurements of Harrison et al. (2015). This is far fewer than 


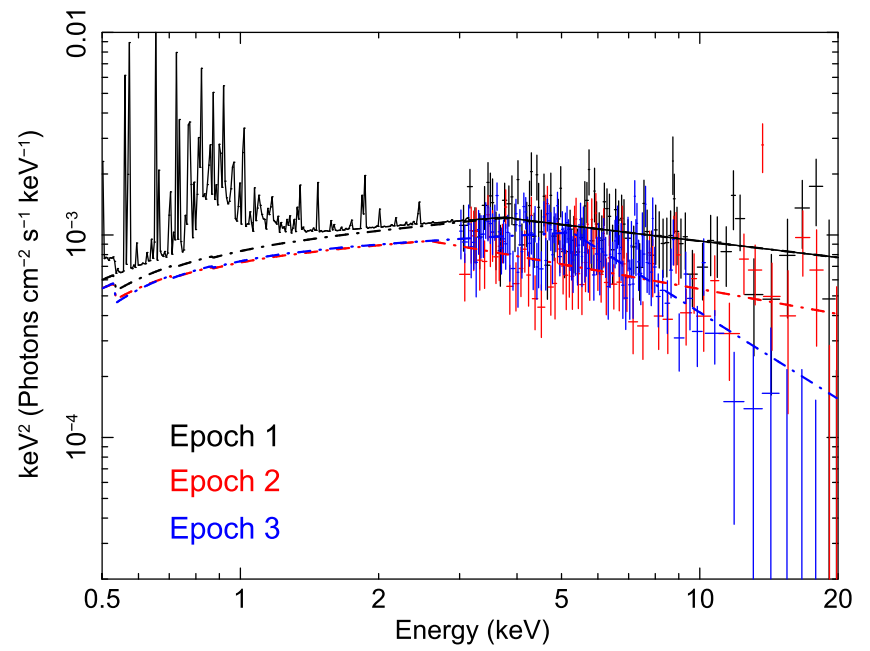

Figure 16. Black line indicates an unfolded model in $E^{2} f(E)$ for the whole galaxy for epoch 1, when an absorbed broken power law and two temperature model is applied. The dashed-dotted lines present the broken power-law component for epochs 1 (black), 2 (red), and 3 (green). The NuSTAR data points are also plotted in this figure to present large statistical uncertainties at harder (especially above $10 \mathrm{keV}$ ) energies.

the total 21 point sources and should not affect our main results. We further note that the Chandra sources have been thoroughly studied using multi-wavelength data and, since any moderate-redshift AGN would have optical/IR counterparts already identified, the remaining point sources identified in our NUSTAR observations are unlikely to be background AGNs.

The remaining 20 sources are thus likely to be associated with M83. Our diagnostics suggest that the majority of the NuSTAR point sources in M83 are consistent with BH binaries in the intermediate-accretion state and NS LMXBs. We note that this could be due to a selection bias. The intermediate state in our diagrams by selection traces the very high state (VHS; Done et al. 2007), which is also referred to as the SPL (see review in Tetarenko et al. 2016), and thus likely our more $\mathrm{X}$-ray luminous sources are in this higher state. The commonality with the intermediate state for lower X-ray luminosity black holes is that the disk and non-thermal components are of comparable magnitude. We also point out that NS LMXBs with high X-ray luminosity on the plot are likely to be $\mathrm{Z}$ sources accreting very close to their Eddington limit.

Fourteen out of the $21 \mathrm{NUSTAR}$ sources have been studied in detail via Chandra spectral analysis in L14, who classified five of them as soft-state accreting BH binaries. This includes source $\mathrm{x} 286$, which is also classified as a BH-XRB in the soft accretion state from our NUSTAR color diagnostics. For the remaining four BH binaries (x321, x248, x284, x403) that were identified as soft-state sources by Chandra, we measured NUSTAR colors that are more consistent with the intermediate state; however, they are still consistent with the soft state within the (large) errors. We also point out that the boundaries of the different states in our diagrams are not sharp.

Differentiating between intermediate and soft accretion states is difficult using only the Chandra band as the spectra are more subtly different in this band (e.g., Figure 9 of Done et al. 2007). NUSTAR has a larger lever arm, akin to the hard $\mathrm{X}$-ray capabilities of RXTE, for detecting the harder components and more sensitively discriminating the intermediate-
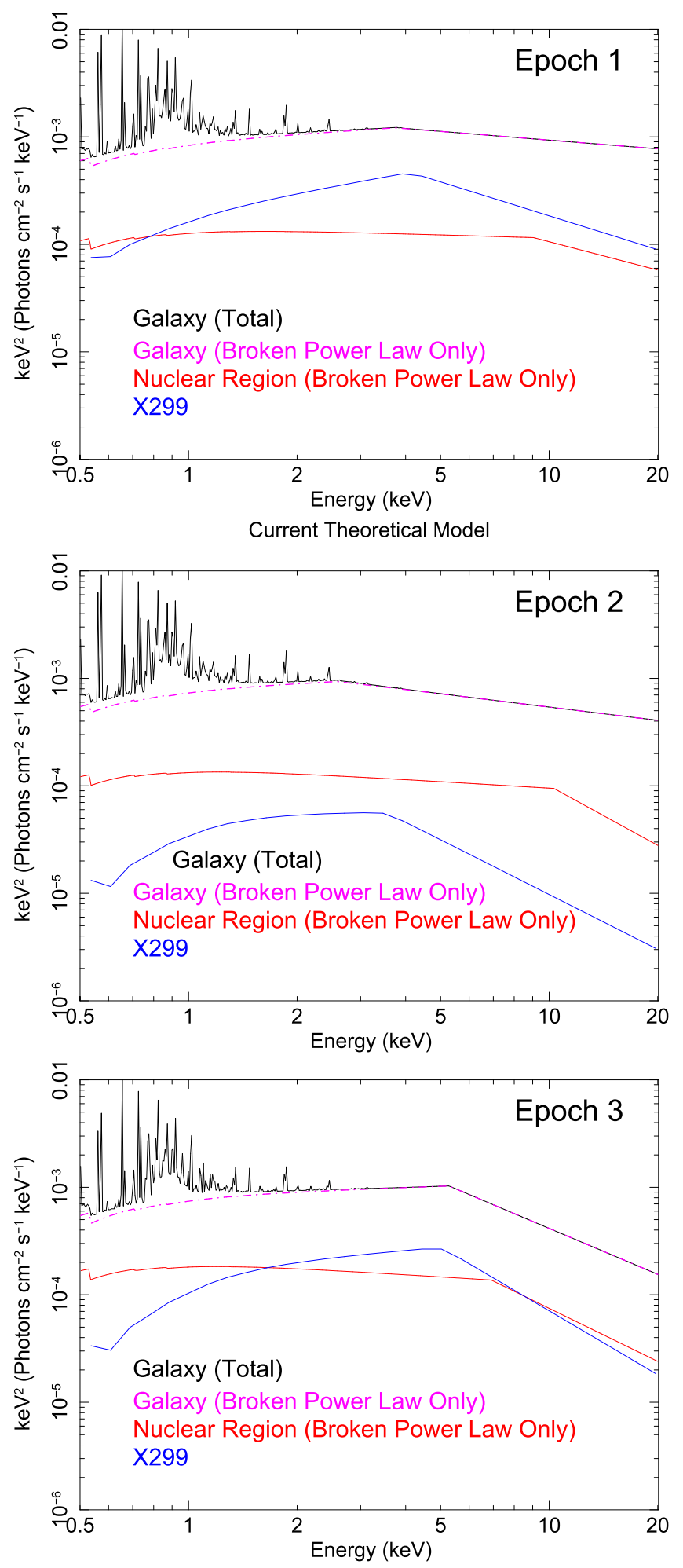

Figure 17. From top to bottom: the unfolded models in $E^{2} f(E)$ for the integrated galaxy including the thermal component (black), nuclear region without thermal component (red), and x299 (blue) for epochs 1, 2, and 3. The integrated galaxy spectra show very similar shapes to the nuclear spectra.

accretion state for these sources. It is possible that detecting objects in this state is thus simply due to a detection limit bias.

The other previously identified ULX, x403 (Immler et al. 1999; Ducci et al. 2013), reached a luminosity of $(2-3) \times 10^{39}$ $\mathrm{erg} \mathrm{s}^{-1}$ in the $0.5-8.0 \mathrm{keV}$ band in January 2014 (see Table 4). 


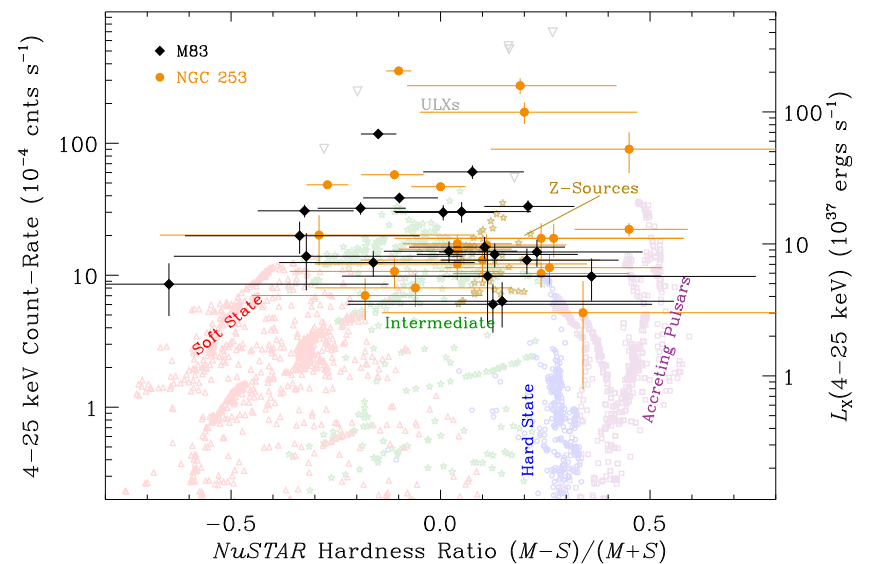

Figure 18. Hardness ratio-intensity plot diagram for M83 (black diamonds) and NGC 253 (orange circles) sources. Other colors and symbols are the same as Figure 10. M83 and NGC 253 have a similar point-source population, such that the majority of sources are $\mathrm{BH}$ binaries in the intermediate-accretion state. Note that NGC 253 has more ULXs.

This source is not very bright in the NuSTAR data, indicating a steeper spectrum. In our spectral fitting of x403 (see Appendix), an absorbed power law is preferred to an absorbed blackbody disk model. Applying an absorbed disk-blackbody plus power-law model to the XMM-Newton spectra (both epochs 1 and 2) resulted in a power-law photon index $\Gamma \sim$ $3-3.5$, and this power-law component dominates even in the softer energy band. The disk-blackbody component is still required above $2 \sigma$ significance. We find that the inner disk temperature $k T_{\text {in }}$ is $\sim 0.9-1.1 \mathrm{keV}$, which is consistent with the $k T_{\text {in }}$ measured from the XMM-Newton data taken in 2013 and 2014 as well as the Chandra observations taken in 2012 (L14), and also is a typical value for $\mathrm{BH}$ binaries.

L14 classified 5 of the $21 \mathrm{NuSTAR}$ sources (x29, x185, x152, $\mathrm{x} 281, \mathrm{x} 193)$ as NSs in XRBs with accretion rates near or above the Eddington limit. Their classification was based on the X-ray luminosity and disk temperature. We note that NS binaries likely have NUSTAR soft colors similar to those of $\mathrm{BH}$ binaries, and it is difficult to distinguish between the two populations in the color-intensity diagnostic alone as shown in the previous section. The NUSTAR intensity and (soft) color of the four sources (x29, x152, x281, x193) are consistent with Galactic NS LMXBs as well as BH binaries with an intermediateaccretion state (Figure 10). The NuSTAR color-color diagnostic may separate two types; however, the hard colors for the three sources are not well constrained. The colors and intensity for $\mathrm{x} 185$ are more consistent with $\mathrm{BH}$ binaries with an intermediate-accretion state. Hard X-ray luminosity is a useful discriminant (see, e.g., Figure 13 of Barret et al. 2000) and is rather unlikely to find NS binaries with $L_{\mathrm{X}} \gtrsim 10^{38} \mathrm{erg} \mathrm{s}^{-1}$ $(4-25 \mathrm{keV})$. It is thus worth exploring if the high-luminosity sources in M83 may indeed be NSs.

From the measured $N u S T A R$ count rates, we estimate that two of the NS binary candidates (x152 and x281) have $L_{X}$ just below $10^{38} \mathrm{erg} \mathrm{s}^{-1}$ in the $4-25 \mathrm{keV}$ band, suggesting that they could be NS binaries. Three other sources have $L_{X} \sim$ a few times $10^{38} \mathrm{erg} \mathrm{s}^{-1}(4-25 \mathrm{keV})$, placing them in the $\mathrm{BH}$ luminosity range. However, L14 mention that x185 is an NS $\mathrm{X}$-ray binary radiating above 2-3 times its Eddington luminosity with an inner accretion disk temperature $k T_{\text {in }}=$ $2.2 \mathrm{keV}$, which corresponds to $r_{\text {in }} \cos \theta \sim 10 \mathrm{~km}$. When we apply a power-law plus disk-blackbody model to the $X M M$ -

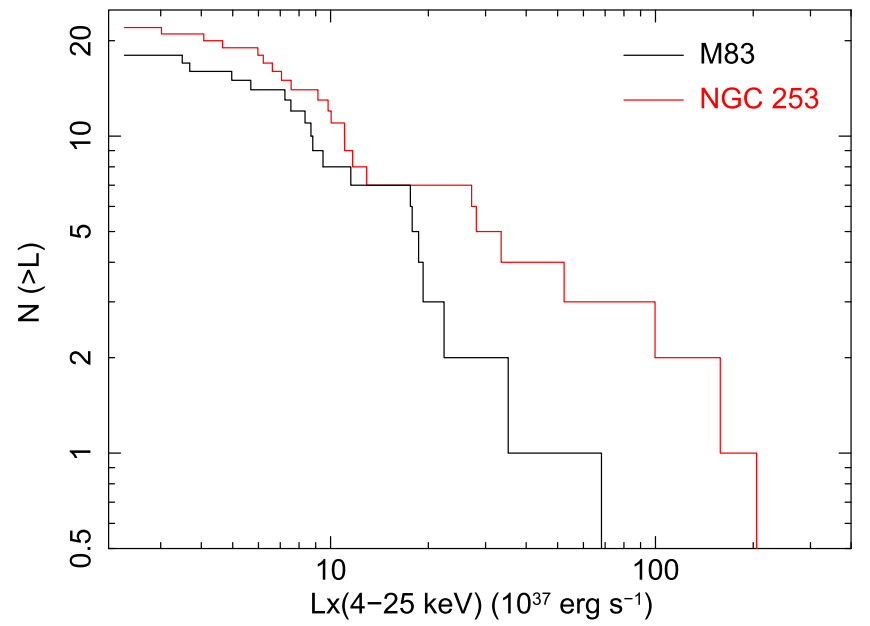

Figure 19. Cumulative $N u S T A R$ point-source XLFs for M83 (black) and NGC 253 (red). The LFs become flatter below $5 \times 10^{37} \mathrm{erg} \mathrm{s}^{-1}\left(0.001 \mathrm{cts} \mathrm{s}^{-1}\right)$, indicating that completeness corrections may need to be taken into account. The slope of the M83 LF is steeper than that of NGC 253.

Newton epoch 1 spectrum of x185, we also obtain a high disk temperature value, $k T_{\text {in }} \sim 2.6 \mathrm{keV}$, although our temperature is not well constrained. As L14 argue, this temperature would be too high for a BH binary. We also applied an absorbed blackbody plus disk-blackbody model, which is a typical spectral model for an NS compact binary. This model fit is comparable to the power-law plus disk-blackbody model fit, resulting in $k T_{\text {in }}=2.2 \mathrm{keV}$ and a blackbody temperature of $\sim 0.3 \mathrm{keV}$. Since it is expected that NSs have $T_{B B} \sim 1-2 \mathrm{keV}$ (e.g., Church et al. 2014), it is likely that we are unable to decouple the NS blackbody from the disk component.

Sometimes, the optical counterpart of X-ray sources help to distinguish the nature of the sources. The $\mathrm{Z}$ sources in Figure 10 are considered to be LXMBs; therefore, we do not expect these systems to contain young stars as their companions. We note that all five sources are located in/near star-forming regions (L14). However, L14 did not find unique optical counterparts for three sources (x152, x185, x281), suggesting that at least these sources could be LMXBs, and possibly $\mathrm{Z}$ sources.

The ULX, $x 299$, is the brightest object in the 4-6, 6-12, and 4-25 keV bands, except for the January 2014 observation when the source was faint $\left(<10^{39} \mathrm{erg} \mathrm{s}^{-1}\right)$. NUSTAR colors and intensity among three epochs confirm that the source moves between the ultraluminous state and high/soft- or intermediateaccretion states, as Soria et al. (2015) suggested from the XMM-Newton spectral analysis. Soria et al. (2015) estimated the mass of the $\mathrm{BH}$ to be $10-20 M_{\odot}$, resulting in $L_{\mathrm{X}} \sim 0.3-2 L_{\text {Edd }}$. The broadband spectral analysis indicates that the bulk of the emission is radiated below $10 \mathrm{keV}$.

\subsection{Comparison to the NGC 253 NuSTAR Source Population}

In this section, we compare the NUSTAR sources detected in NGC 253 and M83, which are star-forming galaxies at comparable distances, with deep NuSTAR coverage, but slightly different star formation properties.

Based on the GALEX FUV and Spitzer $24 \mu \mathrm{m}$ luminosities by Lee et al. (2011) and Dale et al. (2009), and using the SFR equations from Calzetti (2013), we find that M83 and NGC 253 have SFRs of $3.2 M_{\odot} \mathrm{yr}^{-1}$ and $6.1 M_{\odot} \mathrm{yr}^{-1}$, respectively. The stellar mass estimated for M83 and NGC 253 from the 2MASS $K$-magnitudes (Jarrett et al. 2003) and the conversion by Bell 
Table 10

M83 Point Sources: XMM-Newton/Chandra Count Rates and Luminosities

\begin{tabular}{|c|c|c|c|c|c|c|c|}
\hline \multirow{4}{*}{$\begin{array}{l}\text { ID } \\
\text { L14 }\end{array}$} & \multicolumn{2}{|c|}{ Epoch 1} & \multicolumn{2}{|c|}{ Epoch 2} & \multicolumn{2}{|c|}{ Epoch 3} & \multirow[b]{4}{*}{ Variability $^{\mathrm{a}}$} \\
\hline & \multirow{2}{*}{\multicolumn{2}{|c|}{$(0.5-8.0 \mathrm{keV})^{\log L X}$}} & \multirow{2}{*}{\multicolumn{2}{|c|}{ Rate $(0.5-8.0 \mathrm{keV}){ }^{\log L_{\mathrm{X}}}$}} & \multirow{2}{*}{\multicolumn{2}{|c|}{ Rate $(0.5-8.0 \mathrm{keV}){ }^{\log L_{\mathrm{X}}}$}} & \\
\hline & & & & & & & \\
\hline & $\left(10^{-3} \mathrm{ct}^{-1} \mathrm{~s}^{-1}\right)$ & $\left(10^{37} \mathrm{erg} \mathrm{s}^{-1}\right)$ & $\left(10^{-3} \mathrm{ct}^{-1} \mathrm{~s}^{-1}\right)$ & $\left(10^{37} \mathrm{erg} \mathrm{s}^{-1}\right)$ & $\left(10^{-3} \mathrm{ct}^{-1} \mathrm{~s}^{-1}\right)$ & $\left(10^{37} \mathrm{erg} \mathrm{s}^{-1}\right)$ & \\
\hline x 281 & $8.8 \pm 0.8$ & $8.0 \pm 0.7$ & $10.7 \pm 1.0$ & $9.7 \pm 0.9$ & $3.0 \pm 0.3$ & $10.0 \pm 1.1$ & \\
\hline x029 & $13.7 \pm 0.7$ & $12.4 \pm 0.6$ & $9.3 \pm 0.7$ & $8.5 \pm 0.6$ & $6.2 \pm 0.5$ & $20.6 \pm 1.5$ & $\mathrm{v}$ \\
\hline $\mathrm{x} 048$ & $\cdots$ & $\cdots$ & $1.7 \pm 0.7$ & $1.5 \pm 0.6$ & $1.2 \pm 0.2$ & $4.1 \pm 0.7$ & \\
\hline $\mathrm{x} 073$ & $4.3 \pm 0.7$ & $4.0 \pm 0.7$ & $\ldots$ & $\ldots$ & $1.4 \pm 0.2$ & $4.7 \pm 0.8$ & \\
\hline x145 & $6.5 \pm 0.7$ & $5.9 \pm 0.7$ & $7.3 \pm 0.9$ & $6.7 \pm 0.8$ & $2.3 \pm 0.3$ & $7.7 \pm 0.9$ & \\
\hline x165 & $6.1 \pm 0.7$ & $5.6 \pm 0.7$ & $\ldots$ & $\cdots$ & $\cdots$ & $\cdots$ & \\
\hline x 152 & $8.7 \pm 0.9$ & $7.9 \pm 0.8$ & $6.6 \pm 1.1$ & $6.0 \pm 1.0$ & $3.9 \pm 0.4$ & $12.9 \pm 1.2$ & $\mathrm{v}$ \\
\hline x 155 & $3.7 \pm 0.4$ & $3.4 \pm 0.4$ & $1.0 \pm 0.3$ & $0.9 \pm 0.3$ & $\cdots$ & $\cdots$ & \\
\hline x185 & $28.9 \pm 1.1$ & $26.3 \pm 1.0$ & $\ldots$ & $\ldots$ & $11.1 \pm 0.6$ & $36.6 \pm 2.0$ & \\
\hline x193 & $205.6 \pm 2.4$ & $187.1 \pm 2.2$ & $219.3 \pm 3.1$ & $199.6 \pm 2.8$ & $2.5 \pm 0.5$ & $8.1 \pm 1.6$ & \\
\hline $\mathrm{x} 252$ & $\cdots$ & $\cdots$ & $4.5 \pm 0.5$ & $4.1 \pm 0.5$ & $3.3 \pm 0.3$ & $10.9 \pm 1.1$ & \\
\hline $\mathrm{x} 246$ & $21.4 \pm 1.3$ & $19.5 \pm 1.1$ & $41.0 \pm 1.7$ & $37.3 \pm 1.6$ & $0.9 \pm 0.2$ & $3.0 \pm 0.7$ & \\
\hline $\mathrm{x} 233$ & $321.0 \pm 2.9$ & $292.1 \pm 2.6$ & $312.2 \pm 3.8$ & $284.1 \pm 3.4$ & $12.4 \pm 1.0$ & $40.8 \pm 3.4$ & \\
\hline $\mathrm{x} 248$ & $29.7 \pm 1.0$ & $27.0 \pm 1.0$ & $24.6 \pm 1.4$ & $22.4 \pm 1.2$ & $8.9 \pm 0.6$ & $29.1 \pm 1.8$ & \\
\hline $\mathrm{x} 258$ & $8.2 \pm 0.7$ & $7.5 \pm 0.6$ & $\ldots$ & $\ldots$ & $2.8 \pm 0.3$ & $9.3 \pm 1.0$ & \\
\hline $\mathrm{x} 286$ & $30.3 \pm 1.1$ & $27.6 \pm 1.0$ & $48.8 \pm 1.7$ & $44.4 \pm 1.5$ & $16.2 \pm 0.8$ & $53.2 \pm 2.5$ & $\mathrm{v}$ \\
\hline x299 & $252.3 \pm 2.6$ & $229.6 \pm 2.3$ & $57.0 \pm 1.8$ & $51.8 \pm 1.6$ & $57.5 \pm 1.4$ & $189.4 \pm 4.6$ & $\mathrm{v}$ \\
\hline x 284 & $25.2 \pm 1.0$ & $23.0 \pm 0.9$ & $\cdots$ & $\cdots$ & $8.2 \pm 0.5$ & $27.1 \pm 1.8$ & \\
\hline x321 & $28.5 \pm 1.1$ & $25.9 \pm 1.0$ & $35.1 \pm 1.5$ & $32.0 \pm 1.3$ & $10.2 \pm 0.6$ & $33.7 \pm 2.0$ & \\
\hline x366 & $\ldots$ & $\cdots$ & $4.6 \pm 0.8$ & $4.2 \pm 0.7$ & $2.0 \pm 0.3$ & $6.6 \pm 0.9$ & \\
\hline $\mathrm{x} 403$ & $132.7 \pm 1.8$ & $120.8 \pm 1.7$ & $171.6 \pm 2.7$ & $156.2 \pm 2.4$ & $25.9 \pm 0.9$ & $85.3 \pm 3.1$ & $\mathrm{v}$ \\
\hline
\end{tabular}

Notes. The $15^{\prime \prime}$ or $5^{\prime \prime}$ radius aperture is used for XMM-Newton or Chandra data, respectively. Luminosity is calculated using a power-law index of $\Gamma=2$ and Galactic column density, and aperture correction is applied. When a source fell in a chip gap and/or bad pixels, no measurement is listed.

${ }^{a}$ Marked if the luminosity change more than $3 \sigma$ from the average luminosity. x233 and x193 are omitted because of the strong diffuse contamination in the XMMNewton luminosity measurements.

et al. (2003) are $3.3 \times 10^{10} M_{\odot}$ and $5.7 \times 10^{10} M_{\odot}$, respectively. There are 21 total point sources detected in M83 (see Section 5.1) and 22 total point sources in NGC 253 (Wik et al. 2014b), with M83 exhibiting fewer luminous sources (one ULX) than NGC 253 (at least four ULXs). Based on their SFRs and the XLFs of nearby galaxies, we expect 1.5 ULXs for M83 and 3 ULXs for NGC 253 (Swartz et al. 2011; Mineo et al. 2012a).

Figure 18 displays both the M83 and NGC 253 NuSTARdetected sources in the intensity-hardness plot, which is useful for discriminating between $\mathrm{BH}$ accretion states (A. Zezas et al. 2016, in preparation). Most of the sources in NGC 253 and M83 have colors and intensities similar to Milky Way BH binaries in the intermediate and/or very high state. However, we note that, albeit at slightly lower X-ray luminosity, NS LMXBs occupy a similar region of the hardness-intensity diagram as BH-XRBs (Figure 10; also Done et al. 2007). Therefore, most of these sources are also consistent with $\mathrm{Z}$ sources. The color-color diagram shown in Figure 11 constrains the nature of the sources better; however, not all of the XRBs have sufficiently high $\mathrm{S} / \mathrm{Ns}$ in the $12-25 \mathrm{keV}$ band to allow the use of this more discriminatory color-color diagnostic. We note that there has been heightened interest in luminous NS XRB populations recently given the recent discovery by NuSTAR of an extremely luminous pulsar in M82 (e.g., Bachetti et al. 2014). To address the question of the nature of higher $L_{\mathrm{X}}$ non-magnetized NS XRBs, a system like M83 is ideal.

We found one accreting pulsar candidate (no pulsation found) in NGC 253, whereas no such candidates were found in M83. Our detection limit of $\sim 10^{38} \mathrm{erg} \mathrm{s}^{-1}$ is near the upper end of the luminosity for Galactic accreting pulsars. An accreting pulsar with such a luminosity could be either a Type II burst accreting pulsar (Be XRB, the most numerous subclass of HMXBs in the Milky Way and the Magellanic Clouds; Reig 2008) or a Roche lobe overflow pulsar with a supergiant companion like SMC X-1. Be XRBs are generally found to be associated with young bursts of star formation (10-40 Myr; Antoniou et al. 2010, Antoniou \& Zezas, 2016). However, Type II X-ray bursts are rare and short-lived (generally lasting only a few orbital timescales); therefore, detecting such a system points to a host galaxy with a very young stellar population. Contrary to the transient behavior of Be XBRs, Roche lobe overflow systems with a supergiant, which are also very young systems, are persistent, but they are a small fraction of the total population. We note that the persistent nature of the pulsar candidate in NGC 253 may argue against a Be-XRB nature but it could be a supergiant pulsar. We did not find any pulsar binaries in M83 because we assume that the most intense star-forming activity is the nuclear region of the galaxy, which may be confused at the resolution of NuSTAR. In the disk, the XRB populations are much older, and the number of Be XRBs we would expect to observe in a single exposure is very small. The same holds for Roche lobe overflow supergiants.

It has been more than two decades since the first measurement of the strong correlation of X-ray emission from star-forming galaxies with host galaxy properties such as SFR (e.g., David et al. 1992), and it is now relatively well established that much of this strong correlation, particularly at $E>2 \mathrm{keV}$, is due to X-ray binary populations (e.g., Fabbiano 2006 and references therein). One very useful tool for studying binary populations is the XLF. Specifically, it is 
Table 11

M83 Point Sources: Soft X-Ray Spectral Analysis

\begin{tabular}{|c|c|c|c|c|c|c|c|c|c|c|}
\hline $\begin{array}{l}\text { Name } \\
\text { ID }\end{array}$ & Epoch & $\operatorname{Model}^{\mathrm{a}}$ & $\left(10^{21} \mathrm{~cm}_{\mathrm{H}}^{N^{-2}}\right)$ & $\begin{array}{l}\Gamma / k T_{\text {in }} \\
/(\mathrm{keV})\end{array}$ & $\begin{array}{l}\text { Norm } \\
\left(10^{-4}\right)\end{array}$ & C-stat/dof & $\begin{array}{c}\log L_{\mathrm{X}}^{\mathrm{b}} \\
(0.5-8.0 \mathrm{keV}) \\
\left(\mathrm{erg} \mathrm{s}^{-1}\right)\end{array}$ & $\begin{array}{c}k T_{e}^{\mathrm{c}} \\
(\mathrm{keV})\end{array}$ & $\begin{array}{l}\text { Norm } \\
\text { (gas) } \\
\left(10^{-4}\right)\end{array}$ & $f r^{\mathrm{d}}$ \\
\hline \multirow[t]{4}{*}{$\overline{x 281}$} & 1 & PL & $0.4_{-0.4}^{+0.7}$ & $1.47_{-0.19}^{+0.26}$ & $0.06_{-0.01}^{+0.01}$ & $294.6 / 347$ & 38.0 & $\ldots$ & $\ldots$ & $\bar{\ldots}$ \\
\hline & 1 & DB & $0.4_{-0.4}^{+0.4}$ & $1.95_{-0.56}^{+0.93}$ & $1.42_{-1.02}^{+3.07}$ & $302.6 / 347$ & 38.0 & $\ldots$ & $\cdots$ & $\ldots$ \\
\hline & 2 & PL & $0.4_{-0.4}^{+0.5}$ & $1.74_{-0.24}^{+0.27}$ & $0.09_{-0.02}^{+0.02}$ & $216.1 / 275$ & 38.0 & $\cdots$ & $\cdots$ & $\ldots$ \\
\hline & 2 & DB & $0.4_{-0.4}^{+0.3}$ & $1.53_{-0.40}^{+0.58}$ & $4.23_{-2.82}^{+7.86}$ & $230.6 / 275$ & 38.0 & $\cdots$ & $\cdots$ & $\cdots$ \\
\hline \multirow[t]{4}{*}{ x029 } & 1 & PL & $3.6_{-1.3}^{+1.6}$ & $1.65_{-0.25}^{+0.28}$ & $0.18_{-0.05}^{+0.07}$ & $351.4 / 468$ & 38.4 & $\ldots$ & $\ldots$ & $\ldots$ \\
\hline & 1 & DB & $1.5_{-0.8}^{+1.0}$ & $1.98_{-0.38}^{+0.56}$ & $3.15_{-1.82}^{+3.60}$ & $360.4 / 468$ & 38.3 & $\cdots$ & $\cdots$ & $\cdots$ \\
\hline & 3 & PL & $23.5_{-8.5}^{+14.8}$ & $5.01_{-1.44}^{+2.86}$ & $7.28_{-5.51}^{+86.77}$ & $337.3 / 509$ & 38.1 & $\cdots$ & $\cdots$ & $\ldots$ \\
\hline & 3 & DB & $12.1_{-5.0}^{+8.4}$ & $0.55_{-0.21}^{+0.23}$ & $1126.87_{-949.25}^{+9882.93}$ & $336.6 / 509$ & 38.1 & $\cdots$ & $\cdots$ & $\cdots$ \\
\hline \multirow[t]{2}{*}{ x145 } & 1 & PL & $5.8_{-2.3}^{+3.2}$ & $2.38_{-0.43}^{+0.53}$ & $0.20_{-0.08}^{+0.15}$ & $309.5 / 384$ & 38.3 & $\cdots$ & $\cdots$ & $\cdots$ \\
\hline & 1 & DB & $2.5_{-1.4}^{+2.0}$ & $1.16_{-0.25}^{+0.33}$ & $12.46_{-7.78}^{+21.58}$ & $312.1 / 384$ & 38.0 & $\cdots$ & $\ldots$ & $\cdots$ \\
\hline \multirow[t]{2}{*}{ x165 } & 1 & PL & $0.4_{-0.4}^{+1.4}$ & $1.31_{-0.23}^{+0.33}$ & $0.04_{-0.01}^{+0.02}$ & $274.9 / 325$ & 37.9 & $\cdots$ & $\ldots$ & $\ldots$ \\
\hline & 1 & DB & $0.4_{-0.4}^{+0.7}$ & $2.51_{-0.82}^{+0.92}$ & $0.49_{-0.44}^{+1.49}$ & $280.8 / 325$ & 37.9 & $\cdots$ & $\cdots$ & $\cdots$ \\
\hline \multirow[t]{2}{*}{$\mathrm{x} 152$} & 1 & PL & $2.8_{-1.9}^{+4.3}$ & $2.17_{-0.43}^{+0.57}$ & $0.13_{-0.05}^{+0.12}$ & $302.4 / 365$ & 38.1 & $\ldots$ & $\ldots$ & $\ldots$ \\
\hline & 1 & DB & $0.4_{-0.4}^{+2.1}$ & $1.15_{-0.26}^{+0.26}$ & $10.12_{-5.36}^{+19.58}$ & $301.8 / 365$ & 37.9 & $\cdots$ & $\cdots$ & $\cdots$ \\
\hline \multirow[t]{4}{*}{ x185 } & 1 & PL & $2.6_{-0.7}^{+0.8}$ & $1.96_{-0.18}^{+0.19}$ & $0.38_{-0.07}^{+0.08}$ & $479.8 / 629$ & 38.6 & $\ldots$ & $\ldots$ & $\ldots$ \\
\hline & 1 & DB & $0.4_{-0.4}^{+0.5}$ & $1.49_{-0.16}^{+0.15}$ & $14.31_{-3.93}^{+7.57}$ & $490.5 / 629$ & 38.5 & $\cdots$ & $\cdots$ & $\cdots$ \\
\hline & 3 & PL & $3.4_{-2.0}^{+2.3}$ & $1.88_{-0.36}^{+0.39}$ & $0.40_{-0.13}^{+0.22}$ & $371.9 / 509$ & 38.5 & $\ldots$ & $\cdots$ & $\ldots$ \\
\hline & 3 & DB & $1.3_{-1.3}^{+1.6}$ & $1.33_{-0.23}^{+0.34}$ & $23.16_{-13.32}^{+28.05}$ & $359.9 / 509$ & 38.5 & $\cdots$ & $\cdots$ & $\cdots$ \\
\hline \multirow[t]{6}{*}{ x193 } & 1 & PL & $0.7_{-0.2}^{+0.2}$ & $2.58_{-0.14}^{+0.14}$ & $0.98_{-0.11}^{+0.12}$ & $649.9 / 685$ & 39.2 & $0.78_{-0.02}^{+0.02}$ & $0.81_{-0.06}^{+0.07}$ & 0.43 \\
\hline & 1 & DB & 0.4 & $0.41_{-0.02}^{+0.03}$ & $5252_{-1424}^{+1718}$ & $742.1 / 685$ & 39.0 & 0.75 & $0.68_{-0.43}^{+0.43}$ & 0.49 \\
\hline & 2 & PL & $0.9_{-0.3}^{+0.3}$ & $2.75_{-0.21}^{+0.23}$ & $1.17_{-0.18}^{+0.21}$ & $503.7 / 588$ & 39.2 & $0.76_{-0.03}^{+0.03}$ & $1.01_{-0.10}^{+0.12}$ & 0.45 \\
\hline & 2 & DB & $0.4_{-0.4}^{+0.1}$ & $0.42_{-0.03}^{+0.04}$ & $6292.79_{-2037.69}^{+2687.55}$ & $574.3 / 588$ & 39.1 & $0.75_{-0.03}^{+0.03}$ & $0.80_{-0.07}^{+0.07}$ & 0.47 \\
\hline & 3 & PL & 0.4 & $1.95_{-0.36}^{+0.34}$ & $0.14_{-0.05}^{+0.05}$ & $280.1 / 507$ & 38.2 & 0.75 & $0.02_{-0.02}^{+0.02}$ & 0.08 \\
\hline & 3 & DB & 0.4 & $1.28_{-0.25}^{+0.42}$ & $10.1_{-6.6}^{+14.3}$ & $282.9 / 507$ & 38.2 & 0.75 & $0.05_{-0.02}^{+0.02}$ & 0.21 \\
\hline \multirow[t]{4}{*}{ x246 } & 1 & PL & $1.2_{-0.7}^{+0.8}$ & $2.34_{-0.31}^{+0.36}$ & $0.23_{-0.06}^{+0.08}$ & $491.3 / 517$ & 38.3 & $\ldots$ & $\ldots$ & $\ldots$ \\
\hline & 1 & DB & $0.4_{-0.4}^{+0.2}$ & $0.61_{-0.11}^{+0.15}$ & $231.28_{-136.28}^{+289.56}$ & $522.7 / 517$ & 38.1 & $\cdots$ & $\cdots$ & $\cdots$ \\
\hline & 2 & PL & $1.4_{-0.6}^{+0.6}$ & $2.33_{-0.23}^{+0.25}$ & $0.49_{-0.09}^{+0.12}$ & $511.4 / 498$ & 38.6 & $\cdots$ & $\cdots$ & $\cdots$ \\
\hline & 2 & DB & $0.4_{-0.4}^{+0.1}$ & $0.87_{-0.02}^{+0.05}$ & $123.56_{-27.92}^{+34.54}$ & $537.0 / 498$ & 38.5 & $\cdots$ & $\cdots$ & $\cdots$ \\
\hline \multirow[t]{6}{*}{$\mathrm{x} 233$} & 1 & PL & $0.8_{-0.2}^{+0.2}$ & $2.39_{-0.09}^{+0.10}$ & $1.63_{-0.13}^{+0.14}$ & $815.8 / 884$ & 39.4 & $0.78_{-0.02}^{+0.02}$ & $1.24_{-0.07}^{+0.08}$ & 0.39 \\
\hline & 1 & DB & $0.4_{-0.4}^{+0.0}$ & $0.78_{-0.07}^{+0.07}$ & $595.47_{-173.39}^{+283.45}$ & $1105.9 / 884$ & 39.3 & $0.76_{-0.01}^{+0.01}$ & $1.25_{-0.06}^{+0.06}$ & 0.48 \\
\hline & 2 & PL & $0.7_{-0.2}^{+0.2}$ & $2.44_{-0.13}^{+0.14}$ & $1.68_{-0.19}^{+0.21}$ & $663.0 / 741$ & 39.4 & $0.77_{-0.03}^{+0.03}$ & $1.24_{-0.10}^{+0.11}$ & 0.39 \\
\hline & 2 & DB & $0.4_{-0.0}^{+0.0}$ & $0.57_{-0.07}^{+0.11}$ & $2340_{-778}^{+110}$ & $859.3 / 742$ & 39.3 & 0.75 & $1.17_{-0.08}^{+0.08}$ & 0.54 \\
\hline & 3 & PL & $0.9_{-0.8}^{+1.0}$ & $1.87_{-0.26}^{+0.23}$ & $0.43_{-0.12}^{+0.12}$ & $441.49 / 508$ & 38.2 & 0.75 & $0.46_{-0.15}^{+0.31}$ & 0.60 \\
\hline & 3 & DB & $2.3_{-1.6}^{+1.2}$ & $1.97_{-0.53}^{+1.54}$ & $5.53_{-4.7}^{+13.7}$ & $451.5 / 508$ & 39.1 & 0.75 & $5.53_{-0.73}^{+19.2}$ & 0.30 \\
\hline \multirow[t]{6}{*}{$x 248$} & 1 & PL & $7.3_{-1.3}^{+1.5}$ & $2.87_{-0.24}^{+0.25}$ & $1.15_{-0.26}^{+0.36}$ & $518.5 / 600$ & 38.9 & $\cdots$ & $\cdots$ & $\cdots$ \\
\hline & 1 & DB & $3.0_{-0.7}^{+0.8}$ & $0.91_{-0.09}^{+0.09}$ & $122.06_{-41.50}^{+68.67}$ & $508.3 / 600$ & 38.6 & $\cdots$ & $\cdots$ & $\cdots$ \\
\hline & 2 & PL & $4.9_{-1.3}^{+1.7}$ & $2.63_{-0.32}^{+0.37}$ & $0.65_{-0.18}^{+0.29}$ & $342.0 / 451$ & 38.6 & $\ldots$ & $\ldots$ & $\ldots$ \\
\hline & 2 & DB & $1.6_{-0.8}^{+1.0}$ & $0.97_{-0.15}^{+0.18}$ & $67.33_{-33.70}^{+73.68}$ & $342.7 / 451$ & 38.4 & $\ldots$ & $\ldots$ & $\ldots$ \\
\hline & 3 & PL & $9.9_{-2.9}^{+3.6}$ & $3.43_{-0.61}^{+0.76}$ & $1.71_{-0.76}^{+1.70}$ & $333.4 / 509$ & 38.4 & $\ldots$ & $\cdots$ & $\ldots$ \\
\hline & 3 & DB & $4.8_{-2.0}^{+2.3}$ & $0.70_{-0.14}^{+0.17}$ & $351.93_{-221.28}^{+729.67}$ & $325.7 / 509$ & 38.3 & $\cdots$ & $\cdots$ & $\cdots$ \\
\hline \multirow[t]{2}{*}{ x258 } & 1 & PL & $0.4_{-0.4}^{+0.4}$ & $0.94_{-0.24}^{+0.23}$ & $0.04_{-0.01}^{+0.01}$ & $309.1 / 367$ & 38.1 & $\cdots$ & $\ldots$ & $\ldots$ \\
\hline & 1 & DB & $0.4_{-0.4}^{+0.4}$ & $8.90_{-2.85}^{+-8.90}$ & $0.02_{-0.01}^{+0.26}$ & $312.3 / 367$ & 38.2 & $\cdots$ & $\cdots$ & $\cdots$ \\
\hline \multirow[t]{6}{*}{ x286 } & 1 & PL & $2.8_{-0.6}^{+0.6}$ & $2.69_{-0.21}^{+0.23}$ & $0.79_{-0.13}^{+0.16}$ & $495.1 / 552$ & 38.8 & $\cdots$ & $\cdots$ & $\cdots$ \\
\hline & 1 & DB & $0.4_{-0.4}^{+0.3}$ & $0.86_{-0.07}^{+0.05}$ & $143.84_{-30.14}^{+60.64}$ & $483.1 / 552$ & 38.6 & $\cdots$ & $\ldots$ & $\ldots$ \\
\hline & 2 & PL & $2.7_{-0.5}^{+0.6}$ & $2.59_{-0.20}^{+0.22}$ & $0.82_{-0.14}^{+0.17}$ & $417.4 / 526$ & 38.7 & $\cdots$ & $\cdots$ & $\ldots$ \\
\hline & 2 & DB & $0.4_{-0.4}^{+0.2}$ & $0.89_{-0.07}^{+0.04}$ & $139.82_{-28.31}^{+52.10}$ & $404.3 / 526$ & 38.6 & $\cdots$ & $\cdots$ & $\cdots$ \\
\hline & 3 & PL & $5.6_{-1.8}^{+2.2}$ & $2.83_{-0.36}^{+0.42}$ & $1.32_{-0.41}^{+0.70}$ & $427.8 / 509$ & 38.6 & $\ldots$ & $\ldots$ & $\ldots$ \\
\hline & 3 & DB & $1.6_{-1.1}^{+1.3}$ & $0.86_{-0.12}^{+0.14}$ & $180.94_{-86.68}^{+185.19}$ & $417.1 / 509$ & 38.6 & $\cdots$ & $\cdots$ & $\cdots$ \\
\hline
\end{tabular}


Table 11

(Continued)

\begin{tabular}{|c|c|c|c|c|c|c|c|c|c|c|}
\hline $\begin{array}{l}\text { Name } \\
\text { ID }\end{array}$ & Epoch & Model $^{\mathrm{a}}$ & $\begin{array}{c}N_{\mathrm{H}} \\
\left(10^{21} \mathrm{~cm}^{-2}\right)\end{array}$ & $\begin{array}{l}\Gamma / k T_{\text {in }} \\
/(\mathrm{keV})\end{array}$ & $\begin{array}{l}\text { Norm } \\
\left(10^{-4}\right)\end{array}$ & C-stat/dof & $\begin{array}{c}\log L_{\mathrm{X}}^{\mathrm{b}} \\
(0.5-8.0 \mathrm{keV}) \\
\left(\mathrm{erg} \mathrm{s}^{-1}\right)\end{array}$ & $\begin{array}{c}k T_{e}^{\mathrm{c}} \\
(\mathrm{keV})\end{array}$ & $\begin{array}{c}\text { Norm } \\
\text { (gas) } \\
\left(10^{-4}\right)\end{array}$ & $f r^{\mathrm{d}}$ \\
\hline \multirow[t]{6}{*}{ x299 } & 1 & PL & $1.5_{-0.2}^{+0.2}$ & $1.79_{-0.05}^{+0.05}$ & $2.55_{-0.13}^{+0.14}$ & $1182.0 / 1220$ & 39.5 & $\ldots$ & $\ldots$ & $\ldots$ \\
\hline & 1 & DB & $0.4_{-0.4}^{+0.0}$ & $1.40_{-0.04}^{+0.04}$ & $150.84_{-13.73}^{+14.98}$ & $1138.0 / 1220$ & 39.4 & $\ldots$ & $\ldots$ & $\ldots$ \\
\hline & 2 & PL & $2.6_{-0.5}^{+0.5}$ & $2.66_{-0.19}^{+0.20}$ & $0.95_{-0.15}^{+0.18}$ & $435.5 / 534$ & 38.8 & $\cdots$ & $\cdots$ & $\cdots$ \\
\hline & 2 & DB & $0.4_{-0.4}^{+0.2}$ & $0.81_{-0.06}^{+0.03}$ & $227.07_{-38.97}^{+69.33}$ & $416.6 / 534$ & 38.6 & $\cdots$ & $\cdots$ & $\ldots$ \\
\hline & 3 & PL & $2.4_{-0.7}^{+0.7}$ & $1.80_{-0.13}^{+0.13}$ & $1.92_{-0.27}^{+0.32}$ & $512.0 / 509$ & 39.3 & $\cdots$ & $\cdots$ & $\cdots$ \\
\hline & 3 & DB & $0.4_{-0.4}^{+0.4}$ & $1.52_{-0.11}^{+0.09}$ & $75.62_{-14.13}^{+24.64}$ & $501.9 / 509$ & 39.3 & $\ldots$ & $\ldots$ & $\ldots$ \\
\hline \multirow[t]{4}{*}{$\mathrm{x} 284$} & 1 & PL & $5.2_{-0.9}^{+1.0}$ & $3.58_{-0.31}^{+0.34}$ & $1.02_{-0.23}^{+0.32}$ & $436.2 / 450$ & 38.9 & $\cdots$ & $\cdots$ & $\cdots$ \\
\hline & 1 & DB & $1.6_{-0.5}^{+0.6}$ & $0.60_{-0.06}^{+0.07}$ & $458.55_{-183.64}^{+322.34}$ & $416.2 / 450$ & 38.4 & $\cdots$ & $\cdots$ & $\ldots$ \\
\hline & 3 & PL & $4.8_{-2.2}^{+2.5}$ & $3.62_{-0.67}^{+0.83}$ & $1.00_{-0.42}^{+0.85}$ & $295.9 / 509$ & 38.3 & $\ldots$ & $\cdots$ & $\ldots$ \\
\hline & 3 & DB & $0.9_{-0.9}^{+1.6}$ & $0.59_{-0.11}^{+0.12}$ & $455.02_{-255.40}^{+907.46}$ & $284.8 / 509$ & 38.3 & $\ldots$ & $\ldots$ & $\ldots$ \\
\hline \multirow[t]{6}{*}{ x321 } & 1 & PL & $17.9_{-3.0}^{+3.4}$ & $2.91_{-0.28}^{+0.29}$ & $2.44_{-0.77}^{+1.19}$ & $627.3 / 740$ & 39.3 & $\cdots$ & $\cdots$ & $\cdots$ \\
\hline & 1 & DB & $8.7_{-1.8}^{+2.0}$ & $1.16_{-0.11}^{+0.12}$ & $72.28_{-26.96}^{+43.67}$ & $618.0 / 740$ & 38.8 & $\cdots$ & $\cdots$ & $\cdots$ \\
\hline & 2 & PL & $8.4_{-2.4}^{+3.2}$ & $2.37_{-0.29}^{+0.33}$ & $1.03_{-0.32}^{+0.57}$ & $499.6 / 594$ & 38.9 & $\cdots$ & $\ldots$ & $\cdots$ \\
\hline & 2 & DB & $3.5_{-1.0}^{+1.5}$ & $1.28_{-0.14}^{+0.16}$ & $41.01_{-15.99}^{+26.82}$ & $477.0 / 594$ & 38.6 & $\cdots$ & $\cdots$ & $\cdots$ \\
\hline & 3 & PL & $9.6_{-3.5}^{+4.5}$ & $1.98_{-0.43}^{+0.50}$ & $0.63_{-0.27}^{+0.56}$ & $435.4 / 509$ & 38.6 & $\ldots$ & $\ldots$ & $\ldots$ \\
\hline & 3 & DB & $6.1_{-2.3}^{+2.9}$ & $1.43_{-0.30}^{+0.45}$ & $24.13_{-15.68}^{+40.79}$ & $429.8 / 509$ & 38.6 & $\cdots$ & $\cdots$ & $\cdots$ \\
\hline \multirow[t]{6}{*}{$\mathrm{x} 403$} & 1 & PL & $2.7_{-0.2}^{+0.2}$ & $3.02_{-0.10}^{+0.11}$ & $3.01_{-0.23}^{+0.25}$ & $714.1 / 738$ & 39.4 & $\cdots$ & $\ldots$ & ... \\
\hline & 1 & DB & $0.4_{-0.4}^{+0.0}$ & $0.66_{-0.02}^{+0.02}$ & $1393.98_{-167.01}^{+192.36}$ & $762.4 / 738$ & 39.1 & $\cdots$ & $\cdots$ & .. \\
\hline & 2 & PL & $2.3_{-0.2}^{+0.2}$ & $2.58_{-0.09}^{+0.10}$ & $5.05_{-0.40}^{+0.44}$ & $706.8 / 756$ & 39.5 & $\cdots$ & $\cdots$ & $\cdots$ \\
\hline & 2 & DB & $0.4_{-0.4}^{+0.0}$ & $0.84_{-0.03}^{+0.03}$ & $1143.48_{-136.06}^{+159.54}$ & $753.0 / 756$ & 39.4 & $\cdots$ & $\cdots$ & $\cdots$ \\
\hline & 3 & PL & $2.4_{-0.9}^{+1.0}$ & $2.43_{-0.22}^{+0.23}$ & $1.61_{-0.32}^{+0.42}$ & $390.0 / 509$ & 39.0 & $\cdots$ & $\cdots$ & ... \\
\hline & 3 & DB & $0.4_{-0.4}^{+0.2}$ & $0.94_{-0.06}^{+0.02}$ & $242.79_{-32.99}^{+82.58}$ & $392.1 / 509$ & 38.9 & $\ldots$ & $\ldots$ & $\ldots$ \\
\hline
\end{tabular}

Notes.

${ }^{\text {a }}$ PL: power law; DB: disk blackbody.

$\mathrm{b}$ Total intrinsic luminosity.

${ }^{c}$ Assume a solar abundance (Anders \& Grevesse 1989).

${ }^{\mathrm{d}}$ Apec Flux Fraction.

now well established that the normalization of the binary XLF for HMXBs and ULXs scales with SFR (Grimm et al. 2003; Swartz et al. 2011; Mineo et al. 2012a), whereas the LMXB XLF scales with stellar mass (Gilfanov 2004; Kim \& Fabbiano 2010). The XLF for resolved point sources is a powerful tool for characterizing point-source populations in nearby galaxies. The slope of the binary XLF in the $0.5-10 \mathrm{keV}$ bands becomes steeper as the underlying stellar population evolves and the dominant population shifts from HMXBs to LMXBs (e.g., Wu 2001). The high- $L_{\mathrm{X}}$ slope of the Chandra XLF for M83 is found to be steeper $(\gamma=1.38)$ than that of NGC $253(\gamma=0.81$; Kilgard et al. 2002), implying that NGC 253 is likely to be HMXB dominated, whereas M83 (especially in the disk) is LMXB dominated (see also L14). The XLF slopes also differ within M83, showing a flatter slope for the nuclear starburst region and a steeper slope for the disk region (L14).

We constructed NUSTAR XLFs using the $4-25 \mathrm{keV}$ band for both M83 and NGC 253, which are shown in Figure 19. We excluded three (x403, x029, x366) sources in M83 because they were not in the NuSTAR FOV for all three epochs. The remaining sources have roughly similar detection sensitivities in the different epochs (a 30\% difference in the effective exposures). All 22 NuSTAR sources in NGC 253 were in the NUSTAR FOV for all epochs, and thus no sources were excluded.
The flattening below $5 \times 10^{37} \mathrm{erg} \mathrm{s}^{-1}\left(0.001 \mathrm{cts} \mathrm{s}^{-1}\right)$ suggests that incompleteness may be an issue below this limit. We therefore confine our comparisons to above this limit. We clearly see that the high- $L_{\mathrm{X}}$ slope of the M83 NuSTAR LF is steeper than that of NGC 253, which is similar to the soft X-ray LFs for these galaxies with Chandra. A more detailed analysis of the NuSTAR XLF will be presented in a forthcoming paper.

\subsection{Lack of an Active Nucleus?}

The position of the galactic center of M83 has been debated (see Knapen et al. 2010). M83 possesses a nuclear star cluster, which is referred to as the optical nucleus (Gallais et al. 1991). The kinematic center and photometric center are coincident with each other; however, the nuclear star cluster is $\sim 3^{\prime \prime}$ offset from this location (see Figure 1 of Knapen et al. 2010 as well as Sakamoto et al. 2004; Muraoka et al. 2009). This offset suggests that there may be a hidden nuclear mass (i.e., Thatte et al. 2000). However, recent studies (Houghton \& Thatte 2008; Knapen et al. 2010) prefer to adopt the nuclear star cluster as the nucleus.

Our NUSTAR observations detect hard $(>10 \mathrm{keV})$ X-ray emission from the nuclear region; however, our analysis suggests that the emission is likely to be the integrated emission from X-ray binaries instead of the X-ray emission from an obscured AGN based on the steep spectra and the 
resolution of this emission into several sources by Chandra. If M83 contained a highly obscured AGN, such as the Comptonthick AGN in Arp 299 (Ptak et al. 2015), then we would have expected a rising power law to $20-40 \mathrm{keV}$, which we do not observe.

Soria \& Wu (2003) and L14 have shown that there is an X-ray counterpart (x233) coincident with the position of the star cluster nucleus. The averaged spectral slope during 2010 of this X-ray source in the soft X-ray band is a power law with photon index of $\Gamma \sim 1.4$ and $L_{X}$ of $3 \times 10^{38} \mathrm{erg} \mathrm{s}^{-1}$ in the $0.5-8.0 \mathrm{keV}$ band (L14). They suggest that this could be an SMBH with a mass of $1.3 \times 10^{7} M_{\odot}$ (Thatte et al. 2000) accreting with $10^{-7} L_{\text {Edd }}$, but they cannot reject the possibility that it is a compact binary at the location of the nuclear star cluster (e.g., La Parola et al. 2003 for M33, and Yukita et al. 2007 for NGC 2403).

$\mathrm{X} 233$ is also detected in our observations taken in June 2014. By taking a $2^{\prime \prime}$ radius extraction region (331 net counts in the $0.5-8.0 \mathrm{keV}$ band) in the Chandra data in order to reduce the contamination of underlying thermal diffuse emission (note that Tables 10 and 11 use a $5^{\prime \prime}$ radius), an absorbed power-law model gives $\Gamma=2.3_{-0.4}^{+0.7}$ and $N_{\mathrm{H}}=1.3_{-1.0}^{+1.8} \times 10^{21} \mathrm{~cm}^{-2}$ with $L_{\mathrm{X}}^{\text {obs }} \sim 2.8 \times 10^{38} \mathrm{erg} \mathrm{s}^{-1}$ in the $0.5-8.0 \mathrm{keV}$ band. The intrinsic luminosity is about 1.3 times higher than the observed luminosity. The spectral slope reported here is steeper (in June 2014) than that observed in 2010 reported by L14, but the X-ray luminosity in the Chandra band is comparable.

If we extrapolate the spectral shape using a power law with a photon index of $\Gamma=2.3$, obtained from the Chandra spectrum, into the NUSTAR band, then we expect $\times 233$ has $N u S T A R$ count rates of $4 \times 10^{-4} \mathrm{ct} \mathrm{s}^{-1}, 5 \times 10^{-4} \mathrm{ct} \mathrm{s}^{-1}$, and $1 \times 10^{-4} \mathrm{ct} \mathrm{s}^{-1}$ for the 4-6, 6-12, and $12-25 \mathrm{keV}$ bands during epoch 3, respectively. These expected $4-6 \mathrm{keV}$ and $6-12 \mathrm{keV}$ count rates are about a factor of 5 lower than the count rates measured by NUSTAR for the nuclear region, implying that $\mathrm{x} 233$ makes only a small contribution to the nuclear region emission (see Table 3).

We only obtained an upper limit for the observed $12-25 \mathrm{keV}$ count rate, but the expected power-law model count rate in the hard band is below the upper limit of $5.9 \times 10^{-4} \mathrm{ct} \mathrm{s}^{-1}$. The faint sources near $\mathrm{x} 233$, which were resolved in the Chandra image, were not resolved in the NuSTAR images, and these sources may contribute to the measured x233 count rate in the 4-12 keV band. Therefore, the NuSTAR data do not reject that $\mathrm{x} 233$ is M83's SMBH accreting at very low level, whose $0.5-25 \mathrm{keV}$ spectrum is characterized by a single power law with an index of $\Gamma \sim 2.3$. We measure a very low subEddington value for the total X-ray luminosity of the central source. AGNs with these very low levels of $L_{2-10 \mathrm{keV}} / L_{\mathrm{Edd}}$ typically have spectral slopes of $\Gamma=1.4 \pm 0.4$ (e.g., Shemmer et al. 2006; Younes et al. 2011), which is shallower than the constraints we have for the nuclear source. This makes it very unlikely that $\mathrm{x} 233$ is powered by an AGN. We note that the measured X-ray luminosity and spectral values for the nuclear source are also consistent with an X-ray binary.

\section{SUMMARY}

We present the first-ever spatially resolved study of point sources in M83 above $10 \mathrm{keV}$, which was made possible with new NuSTAR observations. NuSTAR's harder energy bandpass constrains and classifies the nature of the resolved sources and accretion states for both BH and NS systems (Wik et al. 2014b; Maccarone et al. 2016). This is only the second deep NuSTAR survey of "resolved" compact object populations in a starforming galaxy outside of the MW following our first study of the nearby starburst NGC 253. Our main results are listed below.

1. Twenty point sources that are likely associated with the galaxy, and are thus XRBs, were found in the NuSTAR images. We found that all of the NUSTAR-detected sources are detected in the extremely deep archival Chandra data available for M83, albeit with a broader range of hard X-ray colors than is seen in the soft X-ray data alone.

Overall, the NUSTAR M83 XRB population differs from that of NGC 253 studied by Wik et al. (2014b), mainly in that M83 posseses fewer luminous sources (fewer ULXs) than NGC 253 in the NUSTAR band, which is expected based on the higher $\operatorname{SFR}\left(6 M_{\odot} \mathrm{yr}^{-1}\right)$ of NGC 253.

2. We have constructed the NuSTAR X-ray binary LF in the 4-25 band $\mathrm{keV}$ for an extragalactic environment for the first time. The slope of the NuSTAR XRB XLF for M83 is steeper than that of NGC 253, which is consistent with previous Chandra results studied at soft X-ray energies. Note that one might expect major differences in the XRB LF at $E>10 \mathrm{keV}$ as we probe to lower $L_{\mathrm{X}}\left(\sim 10^{36} \mathrm{erg}\right.$ $\mathrm{s}^{-1}$ ), which are typical of the obscured HMXB population observed in the Milky Way with INTEGRAL (Lutovinov et al. 2013).

3. We classified XRBs using the NuSTAR point-source color-intensity and color-color diagrams. Based on their NUSTAR colors and intensities, the majority of the sources are likely to be intermediate-accretion state $\mathrm{BH}$ binaries and NS LMXBs (Z-sources). One source is likely to be a soft/high-state BH binary. The known ULX, x299, has luminosity and color (turning over at higher energies) similar to other ULXs studied with NuSTAR. x299 shows long-term variability and possible spectral state transitions, from ULX to soft/intermediate and then back to the ULX state. This state-transition behavior of x299 is similar to what was observed by Soria et al. (2015) using XMM-Newton data. Other sources do not show long-term variability or state transitions.

4. The $0.5-20 \mathrm{keV}$ spectra of the nuclear region indicate no strong reprocessed X-ray emission from the nucleus of M83, suggesting that there is no buried/highly obscured actively accreting SMBH present in this galaxy. We place an upper limit of $\sim 10^{38} \mathrm{erg} \mathrm{s}^{-1}$ on the luminosity of an obscured AGN in the $10-30 \mathrm{keV}$ band. The X-ray point source, x233, found at the location of the nuclear star cluster can be described as a single power law with an index of $\Gamma \sim 2.3$ up to $25 \mathrm{keV}$, a spectral shape that is more consistent with a luminous X-ray binary. However, our data do not rule out the possibility that the source is a low-luminosity AGN.

5. The galaxy-wide spectrum of M83 becomes steeper (with power law index $>2$ ) at harder energies, also seen in other starburst galaxies observed by $N U S T A R$. Either ULX-like sources, intermediate-accretion state $\mathrm{BH}$ binaries, or NS LMXBs (Z-sources) dominate the $10-30 \mathrm{keV}$ emission in these galaxies. This revised $0.5-30 \mathrm{keV}$ SED is of great utility in performing proper k-corrections of 
high- $z$ X-ray constraints on galaxies in the deep Chandra surveys (see, e.g., Lehmer et al. 2016).

We thank the referee for useful comments and suggests that improved this paper. This work was supported under NASA Contract No. NNG08FD60C, and made use of data from the NuSTAR mission, a project led by the California Institute of Technology, managed by the Jet Propulsion Laboratory, and funded by the National Aeronautics and Space Administration. We sincerely thank Karl Forster and Brian Grefenstette for their work on the complicated stray light pattern and for assistance in designing the NuSTAR observational setup for minimizing this contamination. We also thank NUSTAR and Chandra mission planners for making the Chandra and NuSTAR observations simultaneous. We also thank P. Tzanavaris and D. Swartz for helpful discussion. Support for this work was provided by the National Aeronautics and Space Administration through Chandra Award Number GO4-15086Z issued by the Chandra $\mathrm{X}$-ray Observatory Center, which is operated by the Smithsonian Astrophysical Observatory for and on behalf of the National Aeronautics Space Administration under contract NAS8-03060. R.K. acknowledges support from Russian Science Foundation (grant 14-12-01315).

Facilities: Chandra, NuSTAR, XMM-Newton.

\section{APPENDIX \\ XMM-Newton/Chandra POINT SOURCE PROPERTIES}

We briefly summarize the XMM-Newton and Chandra properties for the NUSTAR identified point sources. Table 10 lists the count rates and luminosities in the $0.5-8.0 \mathrm{keV}$ band using the $15^{\prime \prime}$ and $5^{\prime \prime}$ radius aperture for the XMM-Newton and Chandra data, respectively. Aperture correction has been applied. The background is obtained utilizing nearby sourcefree regions. The luminosities are estimated using a power law with a photon index $\Gamma=2$. The variability is flagged if the source flux changes more than $3 \sigma$ from the averaged value among the three epochs.

For the spectral analysis, we use the $0.5-8 \mathrm{keV}$ energy band and applied two models, an absorbed power-law or an absorbed disk-blackbody model (XSPEC model diskbb; Mitsuda et al. 1984). The nuclear sources, $x 233$ and $x 199$, exhibit an excess of soft emission that could be due to hot gaseous emission from the nuclear starburst. Hence, we add an optically thin thermal plasma component (XSPEC apec; Smith et al. 2001) to obtain better fit statistics. The fitting results are tabulated in Table 11.

\section{REFERENCES}

An, H., Madsen, K. K., Westergaard, N. J., et al. 2014, Proc. SPIE, 9144, 1 Anders, E., \& Grevesse, N. 1989, GeCoA, 53, 197

Antoniou, V., \& Zezas, A. 2016, MNRAS, 459, 528

Antoniou, V., Zezas, A., Hatzidimitriou, D., \& Kalogera, V. 2010, ApJL, 716, L140

Bachetti, M., Harrison, F. A., Walton, D. J., et al. 2014, Natur, 514, 202

Bachetti, M., Rana, V., Walton, D. J., et al. 2013, ApJ, 778, 163

Barret, D., Olive, J. F., Boirin, L., et al. 2000, ApJ, 533, 329

Bell, E. F., McIntosh, D. H., Katz, N., \& Weinberg, M. D. 2003, ApJS, 149,289

Boissier, S., Gil de Paz, A., Madore, B. F., et al. 2005, ApJL, 619, L83

Calzetti, D. 2013, in Star Formation Rate Indicators, ed. J. Falcón-Barroso, \& J. H. Knapen (Cambridge: Cambridge Univ. Press), 419

Church, M. J., Gibiec, A., \& Bałucińska-Church, M. 2014, MNRAS, 438, 2784

Church, M. J., Gibiec, A., Bałucińska-Church, M., \& Jackson, N. K. 2012, A\&A, 546, A35
Dale, D. A., Cohen, S. A., Johnson, L. C., et al. 2009, ApJ, 703, 517

David, L. P., Jones, C., \& Forman, W. 1992, ApJ, 388, 82

Dickey, J. M., \& Lockman, F. J. 1990, ARA\&A, 28, 215

Done, C., Gierliński, M., \& Kubota, A. 2007, A\&Ar, 15, 1

Ducci, L., Sasaki, M., Haberl, F., \& Pietsch, W. 2013, A\&A, 553, A7 Fabbiano, G. 2006, ARA\&A, 44, 323

Gallais, P., Rouan, D., Lacombe, F., Tiphene, D., \& Vauglin, I. 1991, A\&A, 243, 309

Gilfanov, M. 2004, MNRAS, 349, 146

Gladstone, J. C., Roberts, T. P., \& Done, C. 2009, MNRAS, 397, 1836

Grimm, H.-J., Gilfanov, M., \& Sunyaev, R. 2003, MNRAS, 339, 793

Harrison, F. A., Aird, J., Civano, F., et al. 2015, arXiv:1511.04183

Harrison, F. A., Craig, W. W., Christensen, F. E., et al. 2013, ApJ, 770, 103

Houghton, R. C. W., \& Thatte, N. 2008, MNRAS, 385, 1110

Immler, S., Vogler, A., Ehle, M., \& Pietsch, W. 1999, A\&A, 352, 415

Jarrett, T. H., Chester, T., Cutri, R., Schneider, S. E., \& Huchra, J. P. 2003, AJ 125,525

Kilgard, R. E., Kaaret, P., Krauss, M. I., et al. 2002, ApJ, 573, 138

Kim, D.-W., \& Fabbiano, G. 2010, ApJ, 721, 1523

King, A. R., Davies, M. B., Ward, M. J., Fabbiano, G., \& Elvis, M. 2001, ApJL, 552, L109

Knapen, J. H., Sharp, R. G., Ryder, S. D., et al. 2010, MNRAS, 408, 797

La Parola, V., Damiani, F., Fabbiano, G., \& Peres, G. 2003, ApJ, 583, 758

Lee, J. C., Gil de Paz, A., Kennicutt, R. C., Jr., et al. 2011, ApJS, 192, 6

Lehmer, B. D., Basu-Zych, A. R., Mineo, S., et al. 2016, arXiv:1604.06461

Lehmer, B. D., Tyler, J. B., Hornschemeier, A. E., et al. 2015, ApJ, in press (arXiv:1505.00789)

Lehmer, B. D., Wik, D. R., Hornschemeier, A. E., et al. 2013, ApJ, 771, 134

Long, K. S., Kuntz, K. D., Blair, W. P., et al. 2014, ApJS, 212, 21

Lutovinov, A. A., Revnivtsev, M. G., Tsygankov, S. S., \& Krivonos, R. A. 2013, MNRAS, 431, 327

Maccarone, T. J., Ykita, M., Hornschemeier, A., et al. 2016, MNRAS, 458, 3633

Madsen, K. K., Harrison, F. A., Markwardt, C., et al. 2015, ApJS, 220, 8

Mineo, S., Gilfanov, M., \& Sunyaev, R. 2012a, MNRAS, 419, 2095

Mineo, S., Gilfanov, M., \& Sunyaev, R. 2012b, MNRAS, 426, 1870

Mitsuda, K., Inoue, H., Koyama, K., et al. 1984, PASJ, 36, 741

Muraoka, K., Kohno, K., Tosaki, T., et al. 2009, PASJ, 61, 163

Murphy, K. D., \& Yaqoob, T. 2009, MNRAS, 397, 1549

Poutanen, J., Lipunova, G., Fabrika, S., Butkevich, A. G., \& Abolmasov, P. 2007, MNRAS, 377, 1187

Ptak, A., Hornschemeier, A., Zezas, A., et al. 2015, ApJ, 800, 104

Rana, V., Harrison, F. A., Bachetti, M., et al. 2015, ApJ, 799, 121

Reig, P. 2008, A\&A, 489, 725

Saha, A., Thim, F., Tammann, G. A., Reindl, B., \& Sandage, A. 2006, ApJS, 165,108

Sakamoto, K., Matsushita, S., Peck, A. B., Wiedner, M. C., \& Iono, D. 2004 ApJL, 616, L59

Shakura, N. I., \& Sunyaev, R. A. 1973, A\&A, 24, 337

Shemmer, O., Brandt, W. N., Netzer, H., Maiolino, R., \& Kaspi, S. 2006, ApJL, 646, L29

Smith, R. K., Brickhouse, N. S., Liedahl, D. A., \& Raymond, J. C. 2001, ApJL, 556, L91

Soria, R., Kuntz, K. D., Long, K. S., et al. 2015, ApJ, 799, 140

Soria, R., Kuntz, K. D., Winkler, P. F., et al. 2012, ApJ, 750, 152

Soria, R., \& Wu, K. 2002, A\&A, 384, 99

Soria, R., \& Wu, K. 2003, A\&A, 410, 53

Stobbart, A.-M., Roberts, T. P., \& Wilms, J. 2006, MNRAS, 368, 397

Strickland, D. K., \& Heckman, T. M. 2007, ApJ, 658, 258

Sutton, A. D., Roberts, T. P., \& Middleton, M. J. 2013, MNRAS, 435, 1758

Swartz, D. A., Soria, R., Tennant, A. F., \& Yukita, M. 2011, ApJ, 741, 49

Tananbaum, H., Gursky, H., Kellogg, E., Giacconi, R., \& Jones, C. 1972, ApJL, 177, L5

Tetarenko, B. E., Sivakoff, G. R., Heinke, C. O., \& Gladstone, J. C. 2016, ApJS, 222, 15

Thatte, N., Tecza, M., \& Genzel, R. 2000, A\&A, 364, L47

Tzanavaris, P., Georgantopoulos, I., \& Georgakakis, A. 2006, A\&A, 454, 447

Walton, D. J., Fuerst, F., Harrison, F., et al. 2013, ApJ, 779, 148

Walton, D. J., Harrison, F. A., Grefenstette, B. W., et al. 2014, ApJ, 793, 21

Wik, D. R., Hornstrup, A., Molendi, S., et al. 2014a, ApJ, 792, 48

Wik, D. R., Lehmer, B. D., Hornschemeier, A. E., et al. 2014b, ApJ, 797, 79

Wu, K. 2001, PASA, 18, 443

Younes, G., Porquet, D., Sabra, B., \& Reeves, J. N. 2011, A\&A, 530, A149

Yukita, M., Swartz, D. A., Soria, R., \& Tennant, A. F. 2007, ApJ, 664, 277 